\title{
Optimality and equilibria in stochastic games
}

\author{
Citation for published version (APA):
}

Thuijsman, F. (1989). Optimality and equilibria in stochastic games. [Doctoral Thesis, Maastricht University]. Rijksuniversiteit Limburg. https://doi.org/10.26481/dis.19890623ft

Document status and date:

Published: 01/01/1989

DOI:

10.26481/dis.19890623ft

Document Version:

Publisher's PDF, also known as Version of record

\section{Please check the document version of this publication:}

- A submitted manuscript is the version of the article upon submission and before peer-review. There can be important differences between the submitted version and the official published version of record.

People interested in the research are advised to contact the author for the final version of the publication, or visit the DOI to the publisher's website.

- The final author version and the galley proof are versions of the publication after peer review.

- The final published version features the final layout of the paper including the volume, issue and page numbers.

Link to publication

\footnotetext{
General rights rights.

- You may freely distribute the URL identifying the publication in the public portal. please follow below link for the End User Agreement:

www.umlib.nl/taverne-license

Take down policy

If you believe that this document breaches copyright please contact us at:

repository@maastrichtuniversity.nl

providing details and we will investigate your claim.
}

Copyright and moral rights for the publications made accessible in the public portal are retained by the authors and/or other copyright owners and it is a condition of accessing publications that users recognise and abide by the legal requirements associated with these

- Users may download and print one copy of any publication from the public portal for the purpose of private study or research.

- You may not further distribute the material or use it for any profit-making activity or commercial gain

If the publication is distributed under the terms of Article $25 \mathrm{fa}$ of the Dutch Copyright Act, indicated by the "Taverne" license above, 
OPTIMALITY AND EQUILIBRIA IN STOCHASTIC GAMES 

OPTIMALITY AND EQUILIBRIA IN STOCHASTIC GAMES

\section{PROEFSCHRIFT}

ter verkrijging van de graad van doctor aan de Rijksuniversiteit Limburg te Maastricht, op gezag van de Rector Magnificus, Prof.dr. F.I.M. Bonke, volgens het besluit van het College van Dekanen, in het openbaar te verdedigen op vrijdag, 23 juni 1989, om 16.00 uur

door

FRANK THUIJSMAN

geboren te Arnhem in 1958 
Promotores: Prof.dr.ir.drs. O.J. Vrieze

Prof.dr. S.H. Tijs

Leden van de beoordelingscommissie:

Prof.dr.ir. A.W.J. Kolen

Dr. R.J.M.M. Does

Prof.dr. J.A. Filar

Prof.dr. H.J. van den Herik

Prof.dr. H.C. Tijms

Financial support was provided by the Netherlands Organization for Scientific Research NWO via the Stichting Mathematisch Centrum (project 10-64-10). 


\section{Acknowledgements}

First of all I wish to thank Koos Vrieze. Without his efforts it would have been impossible for me to write this thesis. Koos was always willing to discuss the problems that I encountered and with his thorough knowledge of stochastic games he always succeeded in providing missing links or in pointing out new directions. Most of the results in this monograph evolved from his cooperation. By treating me not only as a colleague but as a friend, it has always been a great pleasure for me to work with him.

While I was a student I was attracted to the fascinating world of game theory by Stef Tijs. Always incorporating latest results, he very enthousiastically gave, and still gives, many courses on a large variety of topics in game theory. Besides for the many fruitful discussions we had about stochastic games, I thank Stef for giving me the opportunity to develop as a researcher by getting me involved with organizational and educational aspects and by allowing me to present my results at the Nijmegen Game Theory Seminars on several occasions.

I am also very grateful to Annelies, my wife, for her never ceasing support and patience by which she succeeded to ease my mind, again and again. Besides I thank her for all the hours she spent on proof-reading this document.

In Amsterdam at the Centre of Mathematics and Computer Science CWI, Yvonne Samseer did an excellent and accurate job by transforming my manuscript into this satisfactory document. I am also indebted to Wim Aspers for his efficient coordination of the typing process at the CWI. Thanks are also due to the CWI Publications Department for printing this book.

I also want to express my gratitude for the financial support provided by the Netherlands Organization for Scientific Research NWO via the Stichting Mathematisch Centrum. This support enabled me to attend several conferences, where I could exchange ideas with specialists in the field of stochastic games. For their comments I am indebted to all participants of the international workshops on dynamic games, which were held in the previous years in Columbus and Chicago. In particular I like to thank T.S. Ferguson, J A. Filar, J.F. Mertens, A. Neyman, A. Nowak, T. Parthasarathy, T.E.S. Raghavan, T.A. Schultz, S. Sinha and, last bu' not least, S. Sorin. Especially grateful I am to Jerzy Filar for inviting me to visit the University of Maryland at Baltimore and for the pleasant cooperation which resulted from this visit.

Finally I want to thank all my colleagues, friends and family, for their interest in my work. Especially the support of my parents, and of Ruud van Dinten, Christiaan Heij and Hans Peters, has been of great value to me. 



\section{Contents}

\section{Preliminaries}

1.1 Introduction and summary

1.2 The stochastic game model

1.3 Strategies

1.4 Evaluation criteria

1.5 Rewards for stationary strategies

1.6 Playing against a fixed stationary strategy 15

1.7 Zero-sum stocastic games 17

$\begin{array}{lll}1.8 & \text { General-sum stochastic games } & 22\end{array}$

2 Particular initial states in stochastic games

2.1 Introduction 27

2.2 Limit properties for sequences of strategy pairs 28

2.3 Strong initial states in the general-sum case 30

2.4 (E-) Easy initial states in the zero-sum case 37

$3 \quad$ Existence of limiting average equilibria

3.1 Introduction 45

3.2 Finding more strong initial states 45

4 Special classes of stochastic games

4.1 Introduction 59

4.2 Unichain stochastic games 59

4.3 Stochastic games with state independent transitions 60

4.4 Repeated games with absorbing states 62

5 The total reward criterion in zero-sum stochatic games

5.1 Introduction $\quad 69$

5.2 The totall reward criterion 70

5.3 Stochastic games and optimal stationary strategies 75

$\begin{array}{lll}5.4 & \text { The bad match } & 79\end{array}$

5.5 Conclusions 85

6 Stochastic games and mathematical programming

6.1 Introduction 87

6.2 Programs for the $\beta$-discounted reward criterion 88

6.3 Programs for the limiting average reward criterion 90

6.4 Programs for the total reward criterion 95

References $\quad 99$

Author index 103

Subject index 105

Samenvatting $\quad 107$

Curriculum vitae 111 


\section{Chapter 1}

\section{Preliminaries}

\subsection{INTRODUCTION AND SUMMARY}

This monograph deals with two-person stochastic games with finite state and action spaces. The theory of stochastic games started by a paper of Shapley [1953]: 'Stochastic games'. In this fundamental article Shapley combined the dynamic programming model of Bellman [1952, 1957] with the matrix games considered by Von Neumann [1928] and Von Neumann \& Morgenstern [1944].

In the dynamic programming model problems of the following type are considered. At a discrete number of stages in time, a person has to choose one of finitely many actions; that choice will determine an immediate payoff as well as a probability vector according to which a new state is appointed where an action has to be chosen at the next stage. There are finitely many states, each with its own finite action space. The person faces the problem to decide which action choices give the highest income. Here the income is determined by discounting, averaging or, in some special cases, by simply adding all immediate payoffs.

In matrix games two persons, usually called players, face an $m \times n$-matrix with real entries. Simultaneously and independently player 1 has to choose a row and player 2 has to choose a column. The real number in the chosen entry is the amount player 2 has to pay to player 1. Of course, the assumption is made that player 1 wants to maximize the expected payoff and, at the same time, player 2 wants to minimize the expected payoff.

Shapley [1953] combined the features of dynamic programming with those of a matrix game. Thus a stochastic game can be seen as a finite collection of matrix games, one to be played at each stage, where the motion among the matrix games depends at each stage on the current state and on the actions chosen. The collection of stages is assumed to be $N=\{1,2,3, \ldots\}$. The stochastic game is a non-cooperative game, meaning that the players are not allowed to make binding agreements. These stochastic games as examined by Shapley [1953] are zero-sum stochastic games, i.e. one player is paying the other player and the gain of one player is the loss of the other player. In zero-sum games the two players have strictly opposite interests. The question in such games is whether there is a certain amount which player 1 can guarantee to receive (in expectation) regardless of the choices of player 2, while player 2 has a strategy such that he will not need to pay more than that amount (in expectation), regardless of the choices of player 1 . Whenever it exists, this unique amount is called the value of the game and the strategies used by the players to 
guarantee this value are called optimal strategies. If the players can only achieve near-optimality we speak of e-optimal strategies.

If it is not required that one player is paying the other player, then the game is called a general-sum stochastic game. For such a game the states no longer correspond with matrix games but with bimatrix games: in each entry of the matrix there are two real numbers, the first indicating the payoff to row-player 1 , the second indicating the payoff to column-player 2. Now the players need no longer have strictly opposite interests and hence the notions 'value' and "optimality" lose their meaning. In general-sum stochastic games the usual solution concept is that of (e-)equilibria. An (c-)equilibrium is a pair of strategies with the property that neither player can gain (more than $\epsilon(\varepsilon>0)$ ) by unilateral deviation. This concept of equilibrium was introduced by Nash [1951] for bimatrix games; and it is therefore known as Nash-equilibrium. Fink [1964] combined Shapley's (zero-sum) stochastic game model with Nash's (generalsum) solution concept to examine general-sum stochastic games.

In this monograph we shall deal with the general-sum stochastic game model as well as with the zero-sum stochastic game model. The existence of $\epsilon$ equilibria, or of the value and e-optimal strategies, may depend on the initial state. It should be clear that any of the states in a stochastic game can function as the starting state. Just as in dynamic programming it is often useful to consider the problems for the different initial states simultaneously. Thus the value of a stochastic game is in fact a value-vector, where coordinates correspond with the starting states. Likewise an e-equilibrium is a pair of strategies which is an e-equilibrium for all initial states. A further remark to be made is that both the zero-sum and general-sum solution concept depend on the criterion that is used to evaluate the incomes of the players. As in dynamic programming models this criterion can lead to the discounted incomes, the limiting average incomes or the total incomes, where the latter may be quite meaningless if the stochastic game has no specific properties.

In his stochastic game model Shapley required that in each state, for any pair of actions chosen, there is a strictly positive probability that the play terminates. Hence Shapley could derive his results with respect to the total income, since any play would ever terminate with probability 1 . If, in such a terminating stochastic game all stopping probabilities are equal to each other, then examining total incomes in such a game, is equivalent to examining discounted incomes in a related non-terminating stochastic game (cf. Shapley [1953]). Gillette [1957] was the first to examine limiting average incomes in (non-terminating) stochastic games.

In this monograph we consider non-terminating stochastic games. We will deal with all three evaluation criteria. The emphasis however is on stochastic games with respect to the limiting average reward criterion since they have turned out to be quite hard to solve and since the existence of limiting average e-equilibria can be seen as the major open problem in stochastic game theory nowadays. For stochastic games fine solutions are known to exist with respect to the $\beta$-discounted reward criterion, whereas with respect to the total reward criterion similar problems as for the limiting average reward criterion occur. 
More precisely: for the discounted reward criterion solutions exist in terms of stationary strategies, i.e. strategies for which the action choices of the players only depend on the state that is currently visited (cf. Shapley [1953] and Fink [1964]). For the limiting average reward criterion this need not be; the players may have to take into account which actions their opponent used in the past. This was illustrated by an example in Gillette [1957] which has been solved by Blackwell \& Ferguson [1968] using history dependent strategies (cf. example 1.7.4 below). For the total reward criterion history dependent strategies may be required as well (cf. section 5.4). In the example of Gillette [1957], which became known as 'the big match', player 1 has no history independent limiting average e-optimal strategies. So the solution by Blackwell \& Ferguson [1968] of this big match clearly showed that for the limiting average reward criterion history dependent strategies are really indispensable. Unfortunately history dependent strategies have a rather complex structure and often lead to computationall difficulties. Stationary strategies can be seen as the most simple strategies in stochastic games. Any pair of stationary strategies is related with a Markow process on the set of states. This implies, as will be clear in the sequel of this chapter, that for stationary strategies rewards can be computed rather straightforwardly. Hence from the point of view of computations, and hence of potential applications of stochastic games, the class of stationary strategies is particularly interesting. Therefore in literature, as in this monograph, a lot of attention is given to stationary strategies.

Now, knowing that for the limiting average reward criterion, as well as for the total reward criterion, solutions (e-optimal strategies/c-equilibria) may fail to exist if the players are restricted to stationary strategies, it is of special interest to find out what characterizes stochastic games which do have stationary solutions. For the limiting average criterion such a characterization, by means of a system of equations, is presented in chapter 5, due to Vrieze [1987-a]. We present a similar characterization for the existence of stationary total optimal strategies in chapter 5 . In chapter 6, which is based on Filar et al. [1987], we completely characterize the existence of stationary solutions by means of global optima of suitably constructed non-linear mathematical programs. This is done for each of the three evaluation criteria and for zero-sum as well as for general-sum stochastic games. Previously characterizations for the existence of stationary solutions have also been reported in Sobel [1971], Bewley \& Kohlberg [1978], Filar \& Schultz [1986] and Schultz [1987]. So far these characterizations are formulated for zero-sum or general-sum stochastic games with finite state and action spaces without some specific extra structure. Besides, several classes of stochastic games, i.e. stochastic games with a special condition on the payoff and/or transition structure, have been examined for which stationary solutions exist. We mention: unichain/irreducible stochastic games (cf. Gillette [1957], Hoffman \& Karp [1966], Rogers [1969], Sobel [1971], Federgnuen [1978]); recursive games (cf. Everett [1957], Orkin [1972]); single controller stochastic games (cf. Stern [1975], Parthasarathy \& Raghavan [1981], Hordijk \& Kallenberg [1981-b], Filar [1984, 1986], Filar \& Raghavan [1984], Vrieze [1987-a]); stochastic games with perfect information (cf. Gillette [1957]. 
Liggett \& Lippman [1969]); switching control stochastic games (cf. Fillar [1981-b], Filar \& Schultz [1987], Vrieze [1987-a], Vrieze et al. [1983]); stochastic gamess with state independent transitions and separable rewards (cf. Sobel [1981], Parthasaiathy et al. [1984]); stochastic games with additive rewards and additive transitions (cf. Raghavan et al. [1985], Filar \& Schultz [1987]).

For many of these classes there are algorithms known to solve such games. For a survey on algorithms we refer to Raghavan \& Filar [1989].

Although apparently for many classes of stochastic games stationary solutions exist, in general they do not, as was illustrated by the big match. Kohlberg [1974] extended the work of Blackwell \& Ferguson [1968] by showing that for any zero-sum repeated game with absorbing states (cf. section 4.4) the limiting average value exists. Based on the techniques in these papers and using results of Bewley \& Kohlberg [1976] on asymptotic properties of discounted solutions for zero-sum stochastic games, it was derived by Mertens \& Neyman [1981] that for any zero-sum stochastic game the limiting average value exists.

However, as mentioned before, history dependent strategies will be needed to achieve e-optimality. Since we would prefer stationary solutions, it is fortunate to know that in any zero-sum stochastic game there is, for each player, a nonempty set of initial states for which this player has a stationary limiting average optimal strategy. A first proof for this result is given by Tijs \& Vrieze [1986]. In chapter 2 of this monograph we present a new, and more elementary, proof for this result. Besides we give a sufficient condition for which, for all initial states with maximal or minimal limiting average value, both players have stationary limiting average $\epsilon$-optimal strategies.

For the general-sum case a similar result is presented in chapter 2: there is always a non-empty set of initial states for which an 'almost-stationary' limiting average $\epsilon$-equilibrium exists. In chapter 3 this result is extended by formulating sufficient conditions for the existence of an "almost-stationary' limiting average e-equilibrium in any general-sum stochastic game. The existence of limiting average e-equilibria is one of the major remaining problems in stochastic game theory.

The history of general-sum stochastic games started with Fink [1964], who proved the existence of stationary $\beta$-discounted equilibria. Other proofs for this result have been given by Takahashi [1964], Rogers [1969] and Sobel [1971]. The existence of stationary limiting average equilibria has been shown for several classes of stochustic games, most of those mentioned above. Inspired by Sorin [1986] (cf. example 1.8.6 in this chapter), the existence of (history dependent) limiting average c-equilibria for general-sum repeated games with absorbing states was shown by Vrieze \& Thuijsman [1989] using Kohlberg [1974]. In chapter 4 we give a slightly modified proof for this result.

Although stochastic games with just one state, better known as 'repeated games", are part of the model we discuss in this monograph, the theory on such games developed in an other direction than the theory on stochastic games with more than one state. Therefore we do not discuss repeated games in particular in this monograph. For surveys on repeated games we refer to 
Aumann [1981], Mertens [1986] and Sorin [1988],

To finish this brief introduction to stochastic game theory, we want to refer to the surveys on stochastic games by Parthasarathy \& Stern [1977], Raghavan \& Filar [1989], Vrieze [1987-b] and Thujjsman [1987].

We now describe the set up of this monograph.

In the remainder of this chapter we give the formal definitions of the stochastic game model with its solution concepts. Furthermore we formulate the major historic results in this fleld, in view of the topies in this monograph, and we derive several preliminary lemmas and discuss some examples.

In chapter 2 we show that for any general-sum stochastic game there is a non-empty set of initial states for which there exists an almost stationary limiting average $\epsilon$-equilibrium, i.e. limiting average $\epsilon$-equilibrium consisting of stationary strategies amplified with threat-strategies. For zero-sum stochastic games we give an elementary proof for the existence of easy initial states for each player, i.e. starting states for which this player has a stationary limiting average optimal strategy. Tijs \& Vieze [1986] already proved this result, but our proof is significantly simpler. For the set of initial states with maximal or minimal limiting average value, we give a sufficient condition for each player to have stationary limiting average c-optimal strategies. We also show that there may be states which are neither ( $\epsilon$-)easy for player 1 nor for player 2.

In chapter 3 we extend the general-sum results of chapter 2 to formulate sufficient conditions for the existence of a limiting average $\epsilon$-equilibrium (for all starting states). These sufficient conditions are formulated in terms of properties of an arbitrary sequence of stationary $\beta$-discounted equilibria, which without loss of generality can be assumed to converge for $\beta$ going to 1 .

In chapter 4 we show that our results of chapters 2 and 3 imply the existence of limiting average (c)-equilibria for several subclasses: unichain stochastic games (which includes irreducible stochastic games), stochastic games with state independent transitions (SIT), repeated games with absorbing states.

Chapter 5 is devoted to zero-sum stochastic games with the total reward criterion. We show that the totul value may fail to exist, even on the condition that the limiting average value is 0 . On the stronger condition of limiting average value 0 and both players possessing stationary limiting average optimal strategies, history dependent behavior strategies may still be indispensable to the players to achieve total coptimality. This is illustrated by an examplle: "the bad match". We give characterizations for the existence of stationary total optimal strategies (as well as for stationary $\beta$-discounted optimal and limiting average optimal strategies). We relate this total reward criterion with the $\beta$ discounted and the limiting average reward criterion.

Chapter 6 deals with mathematical programs related to stochastic games. With respect to all three evaluation criteria non-linear programs are given that completely characterize the existence of stationary equilibria / (c-)optimall strategies. For the characterization with respect to the total reward criterion we use the assumption that the limiting average reward is 0 for all pairs of stationary strategies. 


\subsection{THE STOCHASTIC GAME MODEL}

\subsubsection{DeFimition}

A stochastic game $\Gamma$ is a 6.tuple $<S,\left\{A_{s}: s \in S\right\},\left\{B_{s}: s \in S\right\}, r^{\|}, r^{2}, p>$, where: $S:=\{1,2, \ldots, z\}, z \in \mathbb{N}$, is the set of states, or state space;

$A_{s}:=\left\{1,2, \ldots, m_{s}\right\}, m_{s} \in \mathbb{N}_{*}$ is the action space of player $I$ in state $s \in S_{s}$

$B_{s}:=\left\{1,2, \ldots, n_{s}\right\}, n_{s} \in \mathbb{N}$, is the action space of player 2 in state $s \in S$;

$r^{k}: \bigcup_{s \in S}\{s\} \times A_{s} \times B_{s} \rightarrow \mathbb{R}$ is the payoff function for player $k \in\{1,2\}$;

$p: \bigcup_{s \in S}\{s\} \times A_{s} \times B_{s} \rightarrow \Delta^{*}$ is the transition probability map,

$$
p(s, i, j)=(p(\mathbb{1} \mid s, i, j), p(2 \mid s, i, j), \ldots, p(z \mid s, i, j)) .
$$

Here $\Delta^{n}:=\left\{a \in \mathbb{R}^{m}: a \geqslant 0, \sum_{1}^{n} a:=1\right\}$, for any $n \in \mathbb{N}$.

\subsubsection{Notation}

In the examples in this monograph stochastic games will be given as a collection of matrices (matrix(1), matrix(2),..., matrix(z)\}, where matrix(s) has size $m_{s} \times n_{s}$ and entry $(i, j)$ of matrix $(s)$ is given as

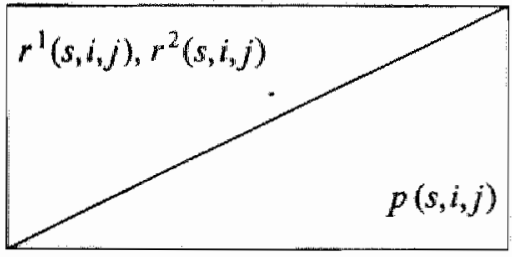

or, in case for some $t \in S$ we have $p(t \mid s, i, j)=1$, as

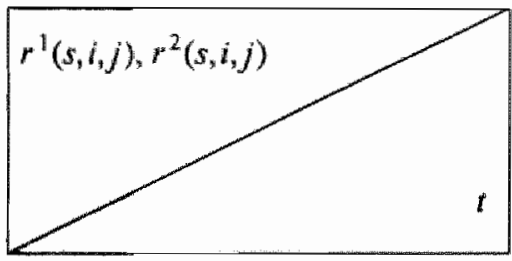

A play of the stochastic game, a 'round' of the game, is assumed to develop in the following way. At each stage $n \in \mathbb{N}$ the play is in precisely one of the states in $S$. The play starts at stage 1 in some state $s_{1} \in S$, the initial state. If at stage $n \in \mathbb{N}$ the play is in state $s_{n} \in S$, then simultaneously and independently, without making binding agreements, player 1 has to choose some $i_{n} \in A_{s_{n}}$ and player 2 has to choose some $j_{n} \in B_{s_{s}}$. Once these choices are made, player 1 receives $r^{1}\left(s_{n}, i_{n}, j_{n}\right)$, player 2 receives $r^{2}\left(s_{n}, i_{n}, j_{n}\right)$ and next the play moves with probability $p\left(s_{n+1} \mid s_{n}, i_{n}, j_{n}\right)$ to state $s_{n+1} \in S$, where the players have to choose 
actions at stage $n+1$.

The players are allowed to randomize over their actions, i.e. in state $s$ player 1 (for example) can use some 'mixed action' $x=\left(x(1), x(2), \ldots, x\left(m_{s}\right)\right) \in \Delta^{m_{s}}$ which is to be interpreted as choosing "pure action" $i \in A_{s}$ with probability $x(i)$. At each stage $n \in \mathbb{N}$ both players know $\Gamma$ as well as the "history" $h_{n}=\left(s_{1}, i_{1}, j_{1}, s_{2}, i_{2}, j_{2}, \ldots, s_{n-1}, i_{n-1}, j_{n-1}, s_{n}\right)$. Neither player knows the mixed actions his opponent has used in the past. Furthermore the assumption is made that both players are interested in maximizing their individual income, which is some kind of evaluation of the payoffs over all stages. Both players are assumed to use the same evaluation criterion (cf. 1.4 below).

Here we already want to make two remarks:

First notice that once a play is started, it never stops, although some stopping-like things may happen as we will see in the sequel. Second it should be observed that with any stochastic game situation there are in fact $z$ games to be considered, one for each starting state. It is often useful to treat these $z$ games simultaneously.

\subsection{Strategies}

Any plan a player uses to play a stochastic game, is called a strategy. So a strategy is a plan that tells a player at all stages, in any state and for any history, what mixed action to use. Within the set of all these strategies one can discern several classes with different complexities. The most simple class of strategies is that of the stationary strategies. A player who uses a stationary strategy has fixed a mixed action in each state, which he uses at any stage the play is in that state, no matter what history preceded.

\subsubsection{DeFINITION}

A stationary strategy for player $l$ is given as an element $x \in X:=\underbrace{x}_{s=1} \Delta^{m}$. A stationary strategy for player 2 is given as an element $y \in Y:=\underset{s=1}{x} \Delta^{n_{2}}$.

These stationary strategies are of fundamental importance in the analysis of stochastic games.

A class of slightly more complex strategies, is that of the Markov strategies. A player who uses a Markov strategy, has fixed a mixed action for each state and stage, to be used at that stage regardless of the history that preceded.

\subsubsection{DeFINITION}

A Markov strategy for player $I$ is a function $f: \mathbb{N} \rightarrow X$.

A Markov strategy for player 2 is a function $g: \mathbb{N} \rightarrow Y$,

The class of Markov strategies for player $I(2)$ is denoted by $F(G)$. 
Observe that stationary strategies are stage independent Markov strategies.

The most complex strategies to be considered in this monograph, are the behavior strategies. A player who uses a behavior strategy will consider the history of the play, in any state and at any stage, to decide what mixed action is to be used. Since this type of strategies is the most general to be considered. (cf. Aumann [1964]), we will often leave out the word behavior.

\subsubsection{DEFINITION}

For $n \in \mathbb{N}$ let $h_{n}:=\left(s_{1}, i_{1}, j_{1}, s_{2}, i_{2}, j_{2}, \ldots, s_{n-1}, i_{n-1}, j_{n-1}, s_{n}\right)$ be the history at stage $n$, i.e. $h_{n}$ is the sequence of states and actions that occurred up to appearance in some state $s_{n}$ at stage $n$.

Let $H_{n}:=\left\{\left(s_{1}, i_{1}, j_{1}, s_{2}, i_{2}, j_{2}, \ldots, s_{n-1}, i_{n-1}, j_{n-1}, s_{n}\right): s_{k} \in S, i_{k} \in A_{s_{k}}, j_{k} \in B_{s_{k}}\right\}$ be the set of histories up to stage $n$.

$A$ (behavior) strategy for player 1 is a function $\pi: \bigcup_{n=1}^{\infty} H_{n} \rightarrow X$.

A (behavior) strategy for player 2 is a function $\sigma: \bigcup_{n=1}^{\infty} H_{n} \rightarrow Y$.

The class of (behavior) strategies for player $1(2)$ is denoted by $\Pi(\Sigma)$.

So we have $X \subset F \subset \Pi$ and $Y \subset G \subset \Sigma$. Although at each stage the current state is part of the history, we say that Markov strategies are history independent strategies.

In the above definitions for strategies the players use mixed actions. The sets of mixed actions contains pure actions, i.e. choosing some row or column with probability 1 . Therefore we can also define pure strategies.

\subsubsection{DEFINITION}

A pure strategy is a strategy for which, for all states, stages and histories, pure actions are used. The set of pure strategies for player $I$ is denoted by $\Pi^{p}$; the set of pure Markov strategies (pure stationary strategies) is denoted by $F^{p}\left(X^{p}\right)$. For player 2 the notations $\Sigma^{p}, G^{p}$ and $Y^{p}$ have a similar meaning.

\subsection{EVALUATION CRITERIA}

As in other game theoretic models the assumption in stochastic games is, that each player wants to maximize his individual income. However, a play of a stochastic game never ends and payoffs occur at all stages. Therefore the players should use some kind of criterion to evaluate those sequences of payoffs in order to decide what strategy they prefer to use. More precisely, each player wants to be able to compare the expected income for several pairs of strategies in order to choose a good strategy. In this monograph we look at the expected income because of the stochastic element caused by the transition probabilities and by the use of mixed actions. 


\subsubsection{DEFINITION}

Let $(\pi, \sigma) \in \Pi \times \Sigma$ be given und let $s \in S$ be the initial state. Define $R^{k}(n)$ as the random variable representing the payoff at stage $n$ to player $k$. Define $E_{\text {ato }}\left[R^{k}(n)\right]$ as the expected payoff at stage $n$ to player $k$ conditional on $s, \pi, 0$.

The above definition is possible because a play starting in state $s$, with the players using $\pi$ and $\sigma$, leads to a well-defined stochastic process on the set of states. For: at stage 1 both $\pi$ and $\sigma$ prescribe some mixed action to be used in state $s$; hence an expected puycif at stage 1 is well-defined for both players, just as are the transitions to the next state. In any new state, at stage 2, strategies $\pi$ and $\sigma$ again prescribe mixed actions to be used, which determines an expected payoff for stage 2, etc.

In this monograph we consider three evaluation criteria: the $\beta$-discounted reward criterion, the limiting average reward criterion and the total reward criterion. Thus a reward to a player for a pair of strategies and an initial state is the evaluated worth to this player of a corresponding sequence of expected payoffs over the stages. We use the word "reward" for an income for some pair of strategies for a whole play, while 'payoff' is always something for just one stage of a play.

A lot of literature in stochastic game theory is on the $\beta$-discounted reward criterion. For the $\beta$-discounted reward criterion stochastic games turn out to have very fine properties and the results for the $\beta$-discounted reward criterion are of fundamental importance for deriving results on the other two criteria. For stochastic games the $\beta$-discounted reward criterion is first mentioned as a remark in Shapley [1953].

\subsubsection{DEFINITION}

Let $\beta \in[0,1)$. The $\beta$-discounted reward to player $k$ for the play starting in state $s$ under $(\pi, \sigma) \in \Pi \times \Sigma$ is given by

$$
\gamma(s, \pi, \sigma):=(1-\beta) \sum_{n=1}^{\infty} \beta^{n-1} E_{s \pi \sigma}\left[R^{k}(n)\right] .
$$

We also use $\gamma \beta \beta(\pi, \sigma):=\left(\gamma_{i}^{k}(1, \pi, \sigma), \gamma_{\beta}^{k}(2, \pi, \sigma), \ldots, \gamma_{\beta}^{k}(z, \pi, \sigma)\right)$.

In this definition the factor $(1-\beta)$ is used to normalize the $\beta$-discounted rewards, because in the sequel we want to relate $\beta$-discounted rewards with limiting average rewards.

Observe that, by the finiteness of the state and action spaces, $E_{s \pi c}\left[R^{k}(n)\right]$ $\in[-M, M]$ for all $s, \pi, \sigma, k, n$, where $M:=\max \left\{\left|r^{k}(s, i, j)\right|: k \in\{1,2\}\right.$, $\left.i \in A_{s}, j \in B_{s}, s \in S\right\}$. Hence $\gamma_{\beta}^{h}(s, \pi, \sigma) \in[-M, M]$ for all $s, \pi, \sigma, k, \beta$. Discounting with a factor $\beta \in(0,1)$ reflects an interest rate $(1-\beta) / \beta$, because an amount $\beta^{n-1} \alpha$ at stage 1 grows to an amount $\alpha$ at stage $n$ under this interest rate. Discounting with factor $\beta$ can also be interpreted as having at each stage a probability $1-\beta$ that the play stops and a probability $\beta$ that the play continues. 
A second important evaluation criterion is the limiting average reward criterion introduced by Gillette [1957]. Most of the results in this monograph are on stochastic games with respect to this criterion.

\subsubsection{DEFINITION}

The limiting average reward to player $k$ for the play starting in state $s$ under $(\pi, \sigma) \in \Pi \times \Sigma$ is given by

$$
\gamma^{k}\left(s_{n} \pi, 0\right):=\liminf _{N \rightarrow \infty} \frac{1}{N} \sum_{n=1}^{N} E_{s \pi \sigma}\left[R^{k}(n)\right] .
$$

We also use $\gamma^{k}(\pi, \sigma):=\left(\gamma^{k}(1, \pi, \sigma), \gamma^{k}(2, \pi, \sigma), \ldots, \gamma^{k}(z, \pi, \sigma)\right)$.

In this definition we use "lim inf" because 'lim' may fail to exist. The 'lim inf' can be interpreted as a pessimistic view of player $k$ in the long run his average income will be at least "lim inf". We could also have chosen 'lim sup' or some Banach limit in the above definition. Of course one can find strategies in a stochastic game such that the limiting average reward for those strategies is different for 'lim inf" and 'lim sup'. However, for stationary strategies 'lim inf' and 'lim sup' lead to the same result.

The third evaluation criterion to be considered in this monograph is that of total rewards, introduced according to the following definition in Thuijsman \& Vrieze [1987] and Vrieze \& Thuijsman [1987].

\subsubsection{DEFINITION}

The total reward to player $k$ for the play starting in state $s$ under $(\pi, \sigma) \in \Pi \times \Sigma$ is given by

$$
\gamma_{T}^{k}(s, \pi, \sigma):=\liminf _{N \rightarrow \infty} \frac{1}{N} \sum_{m=1}^{N} \sum_{n=1}^{m} E_{s \pi \sigma}\left[R^{k}(n)\right] .
$$

We also use $\gamma_{T}^{k}(\pi, \sigma):=\left(\gamma_{T}^{k}(1, \pi, \sigma), \gamma_{T}^{k}(2, \pi, \sigma), \ldots, \gamma_{T}^{k}(z, \pi, \sigma)\right)$.

The use of 'lim inf' in this deflnition will be clear.

Notice that if $\sum_{n=1}^{\infty} E_{s m o}\left[R^{k}(n)\right]$ exists, then it equals $\gamma_{T}^{k}(s, \pi, \sigma)$. For a general stochastic game however, the total rewards will often be $-\infty$ or $+\infty$. The total reward criterion is of special interest in stochastic games for which the limiting average reward is 0 for all, or certain, pairs of stationary strategies. In chapter 5 we discuss this total reward criterion in detail and we examine relations among the three above evaluation criteria.

Observe that the above definitions are all based on the expected payof at the stages. This is possible because the triple $(s, \pi, \sigma)$ determines for each history $h_{n}$, $n \geqslant 2$, a probability of occurrence $\operatorname{Prob}_{s \pi \sigma}^{n}\left(h_{n}\right)$.

However, by the Kolmogorov extension theorem (cf. Kolmogorov [1933]) this sequence of probability measures Prob $_{s \pi r}^{1}()$, Prob $_{s \pi \sigma}^{2}(),. \ldots$ can be extended to a probability measure $\operatorname{Prob}_{s \pi}^{\infty}($.$) on the set of infinite histories, i.e. on the$ 
sequences $\left(s_{1}, i_{1}, j_{1}, s_{2}, i_{2}, j_{2}, \ldots\right)$. Therefore, instead of the above definitions, we could have used alternative criteria defined as

$$
\begin{aligned}
& \tilde{\gamma}_{\beta}^{k}(s, \pi, \sigma):=E_{s \pi 0}\left[(1-\beta) \sum_{n=1}^{\infty} \beta^{n-1} R^{k}(n)\right] ; \\
& \tilde{\gamma}^{k}(s, \pi, \sigma):=E_{s \pi 0}\left[\liminf _{N \rightarrow \infty} \frac{1}{N} \sum_{n=1}^{N} R^{k}(n)\right] ; \\
& \tilde{\gamma}_{T}^{k}(s, \pi, \sigma):=E_{s \pi 0}\left[\liminf _{N \rightarrow \infty} \frac{1}{N} \sum_{m=1}^{N} \sum_{n=1}^{m} R^{k}(n)\right] .
\end{aligned}
$$

For the $\beta$-discounted reward criterion it holds that $\gamma_{\beta}^{k}(s, \pi, \sigma)=\tilde{\gamma}_{\beta}^{k}(s, \pi, \sigma)$ for all $s, \pi, \sigma$; hence also for the solution concepts we will use (cf. section 1.7 and 1.8) $\gamma_{\beta}^{*}($.$) and \gamma_{\beta}($.$) will give the same results, For the limiting average reward cri-$ terion $\gamma^{k}(s, \pi, \sigma)$ not necessarily equals $\bar{\gamma}^{k}(s, \pi, \sigma)$; however for stationary strategies $\gamma^{k}($.$) and \tilde{\gamma}^{k}($.$) lead to the same results. For the total reward criterion$ $\gamma_{T}^{k}(s, x, y)$ not necessarily equals $\tilde{\gamma}_{T}^{k}(s, x, y)$ for stationary strategies $x, y$; mqreover, as will be pointed out in chapter 5 , it is not clear whether or not $\tilde{\gamma}_{T}^{k}($ ) makes any sense at all.

\subsection{REWARDS FOR STATIONARY STRATEGIES.}

As is mentioned above, stationary strategies are the least complex strategies. This is reflected in the fact that for stationary strategies there are fine expressions for the rewards. In this section we introduce those expressions and we give some elementary results needed in the sequel of this monograph.

\subsubsection{DefinItIION}

For a pair of stationary strategies $(x, y) \in X \times Y$ we define:

a) $\operatorname{Car}^{2}(x):={\underset{s}{x}=1}_{2} \operatorname{Car}\left(x_{s}\right)$ with $\operatorname{Car}\left(x_{s}\right):=\left\{i \in A_{s}: x_{s}(i)>0\right\}$, the cartier of $x$ and $x_{s}$ respectively. Car ${ }^{2}(y)$ and $\operatorname{Car}\left(y_{s}\right)$ are defined similarly.

b) $r^{k}\left(x_{3}, y\right):=\left(r^{k}\left(1, x_{1}, y_{1}\right), r^{k}\left(2, x_{2}, y_{2}\right), \ldots, r^{k}\left(z, x_{z}, y_{2}\right)\right)$, with $r^{k}\left(s, x_{s}, y_{s}\right):=\sum_{i=1}^{n} \sum_{j=1}^{n_{s}} x_{s}(i) r^{k}(s, i, j) y_{s}(j)$ being the direct expected payoff to player $k$ in state $s$.

c) $P(x, y)$ is the transition matrix of size $z \times z$. Entry $(s, y)$ of $P(x, y)$ is $p\left(t \mid s, x_{s}, y_{s}\right):=\sum_{i}^{m_{s}} \sum_{j}^{n_{s}} x_{s}(i) p(t \mid s, i, j) y_{s}(j)$, which is the probability of a direct transition from $s$ to $t$ if in state s the players use $x_{3}$ and $y_{s}$.

$P(x, y)$ s denotes row sof $P(x, y)$.

d) $Q(x, y):=\lim _{N \rightarrow \infty} \frac{1}{N} \sum_{n}^{N} P^{n}(x, y)$, where $P^{n}(x, y)$ denotes the $n$ fold matrix product of $P(x, y)$ with itself. $Q(x, y)$ s denotes row $s$ of $Q(x, y)$. 
Observe that $P(x, y)$, for each $(x, y) \in X \times Y$, determines a stochastic process, or Markov chain, on the state space. It is obvious that the (strategy dependent) ergodic structure of such a chain has its impact on the rewards.

\subsubsection{LEMMA}

Let $(x, y) \in X \times Y$ and let $I$ denote the $z \times z$ identity matrix.

a) Entry $(s, t)$ of $P^{n-1}(x, y)$ equals the probability that at stage $n$ the play is in state $t$ if the players use $(x, y)$ and the initial state is $s$.

b) $E_{s x y}\left[R^{k}(n)\right]=P^{n-1}(x, y)_{s} r^{k}(x, y)$.

c) Entry $(s, t)$ of $Q(x, y)$ is the expected average number of visits to state t if the play starts in state $s$ and the players use $(x, y)$.

d) $Q(x, y)_{s}$ equals the unique stationary distribution of the Markov chain which starts in si and is related with $(x, y)$.

e) $Q(x, y) P(x, y)=Q(x, y)$.

f) $(I-\beta P(x, y))$ and $(I-P(x, y)+Q(x, y))$ are non-singular matrices for all $\beta \in[0,1)$. Hence $(I-\beta P(x, y)+Q(x, y))$ is non-singular for $\beta$ clase to 1 .

g) $Q(x, y)=\lim _{\beta \uparrow 1}(1-\beta)(I-\beta P(x, y))^{-1}$.

\section{Proof:}

(a) - (d) follow directly from the definitions; (e) - (g) can be found in Kemeny \& Snell [1960] or in Blackwell [1962].

Observe that (c) and (d) of the above lemma imply that, if $s$ and $t$ are in the same ergodic set of the Markov chain related with $P(x, y)$, then $Q(x, y)_{s}=Q(x, y)_{t}$ and entry $(s, t)$ of $Q(x, y)$ is strictly positive. For $s, t \in S$ with $t$ transient with respect to $P(x, y)$, entry $(s, t)$ of $Q(x, y)$ equals 0 .

\subsubsection{LEMMA}

Let $(x, y) \in X \times Y$ and $\beta \in[0,1)$. Then the following statements hold.

a) $\gamma_{\beta}^{k}(x, y)=(1-\beta) \sum_{n=1}^{\infty} \beta^{n} 1 P^{n-1}(x, y) r^{k}(x, y)$.

b) $\gamma_{\beta}^{k}(x, y)=(1-\beta)(I-\beta P(x, y))^{-1} r^{k}(x, y)$.

c) $\gamma_{\beta}^{k}(x, y)$ is the unique $\alpha^{k} \in \mathbb{R}^{k}$ satisfying $\alpha^{k}=(1-\beta) r^{k}(x, y)+\beta P(x, y) \alpha^{k}$.

\section{Proof:}

By definition $\gamma_{\beta}^{k}(s, x, y)=(1-\beta) \sum_{n=1}^{\infty} \beta^{n-1} E_{s x y}\left[R^{k}(n)\right]$ for all $s \in S$. By lemma 1.5 .2 (b) this implies $\gamma \hat{\beta}(x, y)=(1-\beta) \sum_{n=1}^{\infty} \beta^{n-1} P^{n-1}(x, y) r^{k}(x, y)$. Since $(1-\beta) \sum_{n=1}^{\infty} \beta^{n-1} P^{n-1}(x, y)=(1-\beta)(I-\beta P(x, y))^{-1}$ for any stochastic matrix $P(x, y)$, we have $\gamma_{\beta}^{k}(x, y)=(1-\beta)(I-\beta P(x, y))^{-1} r^{k}(x, y)$. This implies $(I-\beta P(x, y)) \gamma_{\beta}^{k}(x, y)=(1-\beta) r^{k}(x, y)$ and hence $\gamma_{\beta}^{k}(x, y)$ is a solution of $\alpha^{k}=(1-\beta) r^{k}(x, y)+\beta P(x, y) \alpha^{k}$. By the non-singularity of $(I-\beta P(x, y))$ this solution is unique. 


\subsubsection{LEMMA}

Let $(x, y) \in X \times Y, \beta \in[0,1)$ and $\alpha \in \mathbf{R}^{2}$.

a) If $\alpha \leqslant(1-\beta) r^{k}(x, y)+\beta P(x, y) \alpha$, then $\alpha \leqslant \gamma(x, y)$.

b) If $\alpha \leqq(1-\beta) r^{k}(x, y)+\beta P(x, y) \alpha$, then $\alpha \leqq \gamma_{\beta}^{k}(x, y)$.

c) Simitar statements hold when reversing the Inequality signs.

Proof:

If $\alpha \leqq(1-\beta) r^{k}(x, y)+\beta P(x, y) \alpha$, then $(I-\beta P(x, y)) \alpha \lessgtr(1-\beta) r^{k}(x, y)$. Since $(I-\bar{\beta} P(x, y))^{-1}$ is non-negative and each column has at least one positive entry, it follows that $\alpha \lessgtr(1-\beta)(I-\beta P(x, y))^{-1} r^{k}(x, y)=\gamma_{\beta}^{k}(x, y)$.

\subsubsection{LEMMA}

Let $(x, y) \in X \times Y$. Then the following statements hold.

a) $\gamma^{k}(x, y)=\lim _{N \rightarrow \infty} \frac{1}{N} \sum_{n=1}^{N} P^{n-1}(x, y) r^{k}(x, y)$.

b) $\gamma^{k}(x, y)=Q(x, y) r^{k}(x, y)$.

c) $\gamma^{k}(x, y)=\alpha^{k}$ for any puir $\left(\alpha^{k}, \delta^{k}\right) \in \mathbb{R}^{z} \times \mathbb{R}^{z}$ satisfying $\alpha^{k}=P(x, y) \alpha^{k}$ and $\alpha^{k}+\delta^{k}=r^{k}(x, y)+P(x, y) \delta^{k}$.

d) $\gamma^{k}(x, y)=\lim _{\beta \uparrow 1} \gamma_{\beta}^{k}(x, y)$.

Proof:

By definition $\gamma^{k}(s, x, y)=\liminf _{N \rightarrow \infty} \frac{1}{N} \sum_{n=1}^{N} E_{s x y}\left[R^{k}(n)\right]$ for all $s \in S$. Lemma 1.5.2(b) implies $\gamma^{k}(x, y)=\liminf _{N \rightarrow \infty} \frac{1}{N} \sum_{n=1}^{N} P^{n-1}(x, y) r^{k}(x, y)$. It is well-known (cf. Kemeny \& Snell [1960]) that $\lim _{N \rightarrow \infty} \frac{1}{N} \sum_{n=1}^{N} P^{n-1}(x, y)$ exists, and equalls $Q(x, y)$. Hence (a) and (b) hold.

If $\left(\alpha^{k}, \delta^{k}\right) \in \mathbb{R}^{z} \times \mathbb{R}^{z}$ and $\alpha^{k}=P(x, y) \alpha^{k}$ as well as $\alpha^{k}+\delta^{k}=r^{k}(x, y)+P(x, y) \delta^{k}$, then multiplying the second equation with $Q(x, y)$, using lemma 1.5.2 (e), gives $Q(x, y) \alpha^{k}+Q(x, y) \delta^{k}=Q(x, y) r^{k}(x, y)+Q(x, y) \delta^{k}$. Hence $Q(x, y) \alpha^{k}=$ $Q(x, y) r^{k}(x, y)=\gamma^{k}(x, y)$ by (b). Furthermore $\alpha^{k}=P(x, y) \alpha^{k}$ implies $\alpha^{k}=Q(x, y) \alpha^{k}$, so we have $\alpha^{k}=\gamma^{k}(x, y)$. (d) follows directly from lemma 1.5 .2 (g).

\subsubsection{LEMMA.}

Let $(x, y) \in X \times Y$ and let $\alpha, \delta \in \mathbb{R}^{*}$.

a) If $\alpha \leqslant P(x, y) \alpha$ and $\alpha+\delta \leqslant r^{k}(x, y)+P(x, y) \delta$, then $\alpha \leqslant \gamma^{k}(x, y)$.

b) A similar statement holds, when reversing the inequality signs.

\section{Proof:}

$\alpha \leqslant P(x, y) \alpha$ implies $\alpha \leqslant Q(x, y) \alpha$ and likewise $\alpha+\delta \leqslant r^{k}(x, y)+P(x, y) \delta$ implies $Q(x, y) \alpha \leqslant Q(x, y) r^{k}(x, y)$. Hence $\alpha \leqslant Q(x, y) r^{k}(x, y)=\gamma^{k}(x, y)$.

The condition $\alpha+\delta \leqslant r^{k}(x, y)+P(x, y) \delta$ in lemma 1.5.6 can be weakened to: 
$\alpha_{s}+\delta_{s} \leqslant r^{k}\left(s, x_{s}, y_{s}\right)+\sum_{t \in S} p\left(t \mid s_{s}, x_{s}, y_{s}\right) \delta_{t}$, for all states $s$ which are recurrent with respect to (the Markov chain related with) $P(x, y)$.

This is possible because by the fact that for all $s \in S$ and any transient state $t$ entry $(s, t)$ of $Q(x, y)$ equals 0 , we can still derive $Q(x, y) \alpha \leqslant Q(x, y) r^{k}(x, y)$.

\subsubsection{LEMMA}

Let $(x, y) \in X \times Y$ and assume $\gamma^{k}(x, y)=0$ for $k=1,2$. Then the following statements hold.

a) $\gamma_{T}^{k}\left(x_{y} y\right)=\lim _{N \rightarrow \infty} \frac{1}{N} \sum_{m=1}^{N} \sum_{n}^{m} P^{n-1}(x, y) r^{k}(x, y)$.

b) $\gamma_{T}^{k}(x, y)=(I-P(x, y)+Q(x, y))^{-1} r^{k}(x, y)$.

c) $\gamma_{f}^{k}(x, y)=\alpha^{k}$ for any pair $\left(\alpha^{k}, \delta^{k}\right) \in \mathbb{R}^{x} \times \mathbb{R}^{z}$ satisfying $\alpha^{k}=r^{k}(x, y)+P(x, y) \alpha^{k}$ and $\alpha^{k}+\delta^{k}=P(x, y) \delta^{k}$.

d) $\gamma_{T}^{k}(x, y)=\lim _{\beta \uparrow l}(1-\beta)^{-1} \gamma / x(x, y)$.

Proor:

By definition $\gamma_{T}^{*}(s, x, y)=\liminf _{N \rightarrow \infty} \frac{1}{N} \sum_{m=1}^{N} \sum_{n=1}^{m} E_{s x y}\left[R^{k}(n)\right]$ for all $s \in S$. Hence $\gamma_{T}^{k}(x, y)=\liminf _{N \rightarrow \infty} \frac{1}{N} \sum_{m=1}^{N} \sum_{n=1}^{m} P^{n-1}(x, y) r^{k}(x, y)$. Remember that by lemma 1.5.5 (b) we have here that $0=\gamma^{k}(x, y)=Q(x, y) r^{k}(x, y)$. Observe that for each $N \in \mathbf{N}:$

$$
\begin{aligned}
(I-P(x, y) & +Q(x, y))\left(\frac{1}{N} \sum_{m=1}^{N} \sum_{m=1}^{m} P^{n-1}(x, y) r^{k}(x, y)\right) \\
& =\frac{1}{N} \sum_{m=1}^{N} \sum_{n=1}^{m}\left[P^{n-1}(x, y) r^{k}(x, y)-P^{n}(x, y) r^{k}(x, y)\right] \\
& =\frac{1}{N} \sum_{m=1}^{N}\left[r^{k}(x, y)-P^{m}(x, y) r^{k}(x, y)\right] \\
& =r^{k}(x, y)-\frac{1}{N} \sum_{m}^{N} P^{m}(x, y) r^{k}(x, y) .
\end{aligned}
$$

Again using $\gamma^{k}(x, y)=0$ and using lemma 1.5.2 (f) for the non-singularity of $(I-P(x, y)+Q(x, y))$, we derive that $\lim _{N \rightarrow \infty} \frac{1}{N} \sum_{m=1}^{N} \sum_{n=1}^{m} P^{n-1}(x, y) r^{k}(x, y)$ exists and equals $(I-P(x, y)+Q(x, y))^{-1} r^{k}(x, y)$, which proves (a) and (b).

As for (c), suppose that $\alpha^{k}$ and $\delta^{k}$ solve $\alpha^{k}=r^{k}(x, y)+P(x, y) \alpha^{k}$ and $\alpha^{k}+\delta^{k}=P(x, y) \delta^{k}$. Multiplying both sides of the last equation with $Q(x, y)$ gives $Q(x, y) \alpha^{k}=0$ by lemma 1.5 .2 (e). Combining this with the first equation gives that $\alpha^{k}-P(x, y) \alpha^{k}+Q(x, y) \alpha^{k}=r^{k}(x, y)$. Finally the non-singularity of $(I-P(x, y)+Q(x, y))$ implies $\alpha^{k}=(I-P(x, y)+Q(x, y))^{-1} r^{k}(x, y)$ and hence by (b) we have $\alpha^{k}=\gamma k(x, y)$.

In order to show (d) notice that $Q(x, y) \gamma_{\beta}^{k}(x, y)=0$ because $Q(x, y) \gamma_{\beta}^{k}(x, y)=$ 
$Q(x, y)\left[(1-\beta) \sum_{n=1}^{\infty} \beta^{n-1} P^{n-1}(x, y) r^{k}(x, y)\right]=(1-\beta) \sum_{n=1}^{\infty} \beta^{n-1} Q(x, y) r^{k}(x, y)=0$ by $\gamma^{k}(x, y)=0$. Hence by lemma 1.5 .3 (c) we have $\gamma_{\beta}^{k}(x, y)=(1-\beta) r^{k}(x, y)+$ $\beta P(x, y) \gamma_{\beta}^{k}(x, y)-Q(x, y) \gamma_{\beta}^{k}(x, y)$. By the non-singularity of $I-\beta P(x, y)+$ $Q(x, y)$ we have $\gamma_{\beta}^{k}(x, y)=(1-\beta)(I-\beta P(x, y)+Q(x, y))^{-1} r^{k}(x, y)$. Since $I-P(x, y)+Q(x, y)$ is also non-singular we get $\lim _{\beta \uparrow 1}(1-\beta)^{-1} \gamma_{\beta}^{k}(x, y)=$ $(I-P(x, y)+Q(x, y))^{-1} r^{k}(x, y)=\gamma_{T}^{k}(x, y)$ by (b).

\subsubsection{LEMMA}

Let $(x, y) \in X \times Y, a, \delta \in \mathbb{R}^{z}$ and assume $\gamma^{k}(x, y)=0$.

a) If $\alpha \leqslant r^{k}(x, y)+P(x, y) \alpha$ and $\alpha+\delta \leqslant P(x, y) \delta$, then $\alpha \leqslant \gamma_{T}^{k}(x, y)$.

b) A similar statement holds, when one reverses all inequality signs.

\section{ProOF:}

From $\alpha+\delta \leqslant P(x, y) \delta$ we derive $Q(x, y) \alpha+Q(x, y) \delta \leqslant Q(x, y) \delta$ and hence $Q(x, y) \alpha \leqslant 0$. From $\alpha-P(x, y) \alpha \leqslant r^{k}(x, y)$ we derive $P^{n-1}(x, y) \alpha-P^{n}(x, y) \alpha \leqslant$ $P^{n-1}(x, y) r^{k}(x, y)$ for all $n \in \mathbb{N}$. This implies that for all $m \in \mathbb{N}$ we have:

$\alpha-P(x, y)^{m} \alpha=\sum_{n=1}^{m}\left(P^{n-1}(x, y) \alpha-P^{n}(x, y) \alpha\right) \leqslant \sum_{n=1}^{m} p^{n-1}(x, y) r^{k}(x, y)$.

Hence $\alpha-\frac{1}{N} \sum_{m=1}^{N} P^{m}(x, y) \alpha<\frac{1}{N} \sum_{m=1}^{N} \sum_{n=1}^{m} P^{m-1}(x, y) r^{k}(x, y)$ for all $N \in \mathbb{N}$.

Letting $N$ tend to infinity and using $Q(x, y) \alpha \leqslant 0$ we obtain $\alpha \leqslant \gamma_{T}^{k}(x, y)$.

\subsection{Playing against a FIXEd stationary strategy}

In any stochastic game both players want to maximize their individual rewards. Since they cannot muke binding agreements they do not know what strategy there opponent is going to use. Nevertheless each player should hope that the strategy he chose is a best reply against the strategy of his opponent, otherwise a better strategy could have been used. Therefore it is of interest to examine what happens if the opponent fixes a strategy. For the objectives in this monograph it is sufficient to consider what happens if the opponent fixes a stationary strategy.

\subsubsection{DeFINITION}

Let $y \in Y$ and $\beta \in[0,1)$.

A $\beta$-discounted best reply for player $l$ against $y$ is a strategy $\pi^{*} \in \Pi$ for which $\gamma_{\beta}^{\mathrm{l}}\left(\pi^{*}, y\right) \geqslant \gamma_{\beta}^{\mathrm{l}}(\pi, y)$ for all $\pi \in \mathrm{II}$. Limiting average best reply and total best reply are defined analogously. 
The next lemma follows from Hordijk et al. [1983] and from Blackwell [1962] and we will often use it for our analysis of stochastic games.

\subsubsection{LEMMA}

Let $y \in Y$ and $\beta \in[0,1)$.

There exists a pure stationary strategy $x^{*} \in X^{p}$ such that $\gamma_{\beta}^{1}\left(x^{*}, y\right) \geqslant \gamma_{\beta}^{1}(\pi, y)$ for all $\pi \in \Pi$. Similarly, there exists a pure stationary best reply for player I against $y$ in the limiting average case.

Hordijk et al. [1983] show that player I cannot do better in the stochastic game against $y$ than to play optimal in the related Markov decision process, which we call $M D P(y)$.

\subsubsection{Definition}

A Markov decision process is a stochastic game where one of the players has only one action available in all stutes. For a stationary strategy $y$ in a stochastic game $\Gamma$, the Markov decision process MDP(y) is the stochastic game $\Gamma^{*}$ with $S^{*}:=S$, $A_{s}^{*}:=A_{s}, \quad B_{s}^{*}:=\{1\}, r^{*}(s, i, 1):=r\left(s, i, y_{s}\right), P^{*}(t \mid s, i, 1):=p\left(t \mid s, i, y_{s}\right)$ for all $i \in A_{s}^{*}$, $s \in S^{*}$.

For Markov decision processes Blackwell [1962] has shown the existence of pure stationary optimal strategies for the $\beta$-discounted reward criterion as well as for the limiting average reward criterion. Combining this with the result of Hordijk et al. [1983] gives lemma 1.6.2.

\subsubsection{LEMMA}

Let $y \in Y$ and $\beta \in[0,1)$.

Let $x^{*} \in X$ be a stationary $\beta$-discounted best reply against $y$. Then:

a) $\gamma_{\beta}^{1}\left(x^{*}, y\right)=(1-\beta) r^{1}\left(x^{*}, y\right)+\beta P\left(x^{*}, y\right) \gamma_{\beta}^{1}\left(x^{*}, y\right)$ $(1-\beta) r^{1}(x, y)+\beta P(x, y) \gamma_{\beta}^{1}\left(x^{*}, y\right)$ for all $x \in X$.

b) $\gamma_{\beta}\left(x^{*}, y\right)=(1-\beta) r^{1}\left(x^{p}, y\right)+\beta P\left(x^{p}, y\right) \gamma \beta\left(x^{*}, y\right)$ for all $x^{p} \in X^{p}$ with $\operatorname{Car}^{2}\left(x^{p}\right) \subset \operatorname{Car}^{*}\left(x^{*}\right)$.

c) $\gamma_{\beta}^{1}\left(x^{*}, y\right)=\gamma_{\beta}^{1}\left(x^{p}, y\right)$ for all $x^{p} \in X^{p}$ with $\operatorname{Car}^{2}\left(x^{p}\right) \subset \operatorname{Car}^{2}\left(x^{*}\right)$.

\section{Proof:}

The equality sign in (a) follows from lemma 1.5.3 (c).

The inequality sign in (a) follows from the fact that $x^{*}$ is a $\beta$-discounted best reply against $y$.

From (a) it follows that for each $s \in S$ :

$$
\begin{aligned}
\gamma_{\beta}^{1}\left(s, x^{*}, y\right) & =(1-\beta) r^{1}\left(s, x_{s}^{*}, y_{s}\right)+\beta \sum_{i=1}^{z} p\left(t \mid s, x_{s}^{*}, y_{s}\right) \gamma_{\beta}^{\prime}\left(t, x^{*}, y\right) \\
& =\sum_{i \in A_{s}} x_{s}^{*}(i)\left[(1-\beta) r^{\prime}\left(s, i, y_{s}\right)+\beta \sum_{t=1}^{z} p\left(t \mid s, i, y_{s}\right) \gamma_{\beta}^{1}\left(t, x^{*}, y\right)\right] \\
& \leqslant \sum_{i \in A_{s}} x_{s}^{*}(i) \gamma \beta\left(s, x^{*}, y\right)=\gamma \downarrow\left(s, x^{*}, y\right) .
\end{aligned}
$$


Hence $\gamma_{\beta}^{l}\left(s, x^{*}, y\right)=(1-\beta) r_{s}^{l}\left(s, i, y_{s}\right)+\beta \sum_{t=1}^{z} p\left(t \mid s, i, y_{s}\right) \quad \gamma \beta\left(t, x^{*}, y\right)$ for all $i \in \operatorname{Car}\left(x_{s}^{*}\right)$, which proves (b).

Now (c) follows from (b) and from lemma 1.5 .3 (c).

An analogue of lemma 1.6 .4 (c) does not hold for the limiting average case. Consider for instance the following example, where player 2 has only one (trivial) strategy: $y$. We show that a stationary strategy within the carrier of a stationary limiting average best reply against $y$, does not need to be a limiting average best reply against $y$ itself.

\subsubsection{EXAMPLE}

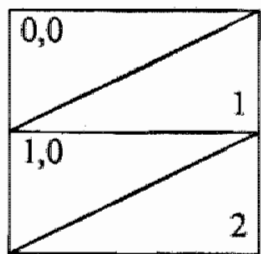

State 1

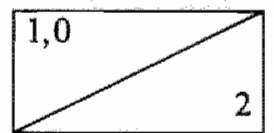

State 2

Stationary strategies for player 1 in the above game, are fully determined by the mixed action which player $\mathbb{1}$ uses in state 1 . So $X=\{(p, 1-p): p \in[0,1]\}$. It is easy to see that $(1 / 2,1 / 2)$ is a stationary limiting average best reply against $y$, giving player 1 limiting average reward $(1,1)$. Although the pure stationary strategy $(1,0)$ is clearly contained in the carrier of $(1 / 2,1 / 2)$, it is not a limiting average best reply against $y$, for $\gamma^{1}((1,0), y)=(0,1)$.

\subsection{ZERO-SUM STOCHASTIC GAMES}

Shapley [1953] started the theory of stochastic games. In his model the payoffs to player 1 are the losses of player 2, i.e. $r^{2}(s, i, j)=-r^{1}(s, i, j)$ for all $s, i$ and $j$. A stochastic game with this property is called a zero-sum stochastic game. Since in a zero-sum stochastic game the players have strictly opposite interests, player 1, who wants to maximize his reward, can expect that player 2 wants to minimize that same reward. We assume that player 1 is interested in maximiz ing his guaranteed expected reward, i.e. player 1 would prefer to use a strategy $\pi^{*}$ such that inf $\gamma_{\beta}^{\prime}\left(\pi^{*}, \sigma\right)>\inf _{\sigma} \gamma_{\beta}^{1}(\pi, \sigma)$ for all $\pi \in \Pi$, in the $\beta$-discounted case (or similarly for the other criteria). So player 1 is interested in $\sup _{\pi}$ inf $\gamma_{\beta}(\pi, \sigma)$. Likewise we assume that player 2 wants to minimize the reward to player 1 and is interested in inf $\sup _{\pi} \gamma(\pi, \sigma)$, the coordinatewise minimal level for which player 2 can guarantee that the reward to player 1 will not be greater (up to some $\epsilon>0)$. It is easy to see that 'sup inf' $\leqslant$ 'inf sup' because: inf $\gamma_{\beta}^{\prime}\left(\pi^{*}, a\right) \leqslant$ 
$\gamma_{\beta}^{1}\left(\pi^{*}, \sigma^{*}\right)$ for all $\pi^{*}, \sigma^{*}$, implies that $\sup _{\pi} \inf _{\sigma} \gamma_{\beta}(\pi, \sigma) \leqslant \sup _{\pi} \gamma_{\beta}^{l}\left(\pi, \sigma^{*}\right)$ for all $\sigma^{*}$ and hence $\sup _{\pi} \inf _{\sigma} \gamma_{\beta}^{\prime \prime}(\pi, \sigma)<\inf _{\sigma} \sup _{\pi} \gamma_{\beta}^{\pi}(\pi, \sigma)$. If 'sup inf $=$ "inf sup" then we call this number the value of the stochastic game.

\subsubsection{DEFINITION}

a) If for a zero-sum stochustic game there exists, for $\beta \in[0,1)$, a $v_{\beta}^{1} \in \mathbb{R}^{z}$ such that $\sup _{\pi} \inf _{\sigma} \gamma_{\beta}(\pi, \sigma)=v_{\beta}^{1}=\inf _{\sigma} \sup _{\pi} \gamma_{\beta}(\pi, \sigma)$, then $v_{\beta}^{\mathbb{1}}$ is called the $\beta$ discounted value of the stochastic game.

b) If the value is $v$, then player 1 has, for each $\epsilon>0$, a strategy $\pi_{6}$ such that $\gamma_{\beta}^{1}\left(\pi_{t, j} \sigma\right) \geqslant \nu_{\beta}^{\prime}-\epsilon 1_{z}$ for all $\sigma \in \Sigma$. Such a strategy $\pi_{\varepsilon}$ is called a $\beta$-discounted c-optimal strategy for player 1. A $\beta$-discounted optimal strategy for player 1 is a strategy $\pi^{*}$ for which $\gamma_{\beta}^{1}\left(\pi^{*}, a\right) \geqslant \nu_{\beta}^{1}$ for all $\sigma$.

A similar definition holds for ( $\mathrm{E}$-)optimal strategies of player 2.

c) For the limiting average reward case and the total reward case ( $\epsilon$-)optimal strategies and value, $v$ resp. $\nu_{T}^{1}$, are defined analogously.

In zero-sum stochastic games the players at each stage face a kind of matrix game. Therefore it may not be surprising that the following theorem by Von Neumann [1928], which we present without proof, is very valuable for stochastic games.

\subsubsection{THEOREM}

For any real matrix $A=\left[a_{i j}\right]_{1}^{m}{ }^{m}, j^{n}=1$, there exist $x^{*} \in \Delta^{m}$ and $y^{*} \in \Delta^{n}$ such that for all $x \in \Delta^{m}$ and $y \in \Delta^{n}: x^{*} A y \geq x^{\prime} A y^{*} \geq x A y^{*}$.

The mixed actions $x^{*}$ and $y^{*}$ ure called optimal mixed actions, for player 1 and player 2 respectively, in the matrix game $A$. The number $x^{*} A y^{*}$ is called the value of $A$, denoted by val( $A)$ or by val $\left.a_{i j}\right]$. This value of $A$ is uniquely determined.

The importance of this theorem for stochastic games already occurs in the seminal paper on stochastic gumes by Shapley [1953], who examined stopping stochastic games. For stochastic games with the $\beta$-discounted reward criterion Shapley's results imply the following:

\subsubsection{THEOREM}

For any $\beta \in[0,1)$ and any zero-sum stochastic game:

a) The $\beta$-discounted value $\nu_{\beta}^{1}$ exists and both players have stationary $\beta$ discounted optimal strategies.

b) $\quad v d$ is the unique solution $\alpha \in \mathbb{R}^{z}$ of the 'Shapley-equation': $\left.\alpha_{s}=\operatorname{val}\left[(1-\beta) r^{1}(s, i, j)+\beta \sum_{i=1}^{\sum} p(t \mid s, i, j) \alpha_{t}\right]_{i=1, j=1}^{m_{s}}=: \operatorname{val}(A\}_{\beta}(\alpha)\right), s \in S$.

c) A stationary strategy $x^{*}\left(y^{*}\right)$ for player $1(2)$ is $\beta$-discounted optimal if $x_{s}^{*}$ $\left(y_{s}^{*}\right)$ is an optimal mixed action for player $I(2)$ in the matrix game $A_{\beta}^{l s}\left(v_{\beta}^{1}\right)$ for each $s \in S$. 
It is well-known that (c) of the above theorem is also valid when we replace if by 'if and only if". This follows for example from the results of Vrieze \& Tijs [1980], who showed that for each player the set of stationary $\beta$-discounted optimal strategies is the Cartesian product of the sets of optimal mixed actions in the matrix games $A g(v b), s \in S$.

Gillette [1957] introduced the limiting average reward criterion for stochastic games. With respect to this criterion stochastic games turned out to have a more difficult nature than for the $\beta$-discounted reward criterion. Gillette [1957] gave the following example for which it was not clear for several years, whether or not the limiting average value did exist.

\subsubsection{EXAMPLE (the big match)}

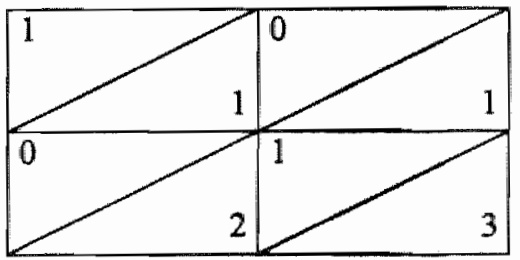

State 1

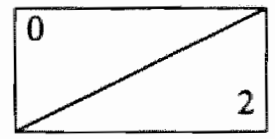

State 2

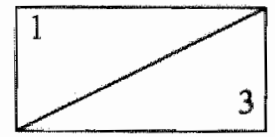

State 3

In this zero-sum stochastic gume, only player l's payoffs are given (cf. 1.2.2). Of course state 1 is the interesting state in this stochastic game and for both players strategies are determined by the mixed actions used in state $\mathbb{1}$. The remarks below illustrate the beauty of this big match, which was solved by Blackwell \& Ferguson [1968].

a) With respect to Markov strategies (cf. 1.3.2) one finds that:

$\sup _{f \in F} \inf _{g \in G} \gamma^{1}(1, f, g)=0$ whereas $\inf _{g \in G} \sup _{f \in F} \gamma^{\Perp}(1, f, g)=1 / 2$.

Hence the limiting average value would not exist if the players were restricted to Markov strategies.

b) Allowing all strategies one finds that:

$$
\sup _{\pi \in f} \inf _{\sigma \in \Sigma} \gamma^{1}(1, \pi, a)=\inf _{0 \in \mathbb{Z}} \sup _{\pi \in \mathbb{H}} \gamma^{1}(1, \pi, a)=1 / 2 \text {. }
$$

So the limiting average value $v^{1}=(1 / 2,0,1)$.

c) For player 2 the stationury strategy determined by using the mixed action $(1 / 2,1 / 2)$ in state 1 , is limiting average optimal. Player 1 has no limiting average optimal strategy and only history dependent limiting average optimal strategies.

If we consider the above stochastic game with respect to the $\beta$-discounted reward criterion then we find by solving the Shapley-equation (cf. 1.7.3) that $v_{\beta}^{1}=(1 / 2,0,1)\left(=\nu^{1}\right)$ for all $\beta \in[0,1)$, while the unique stationary $\beta$-discounted optimal strategies are $x^{\beta}=(1 /(2-\beta),(1-\beta) /(2-\beta))$ and $y^{\beta}=(1 / 2,1 / 2)$ for player 1 and player 2 respectively. 
It should be observed that, with respect to $P\left(x^{\beta}, y^{\beta}\right)$ state 1 is transient, whereas state 1 is recurrent with respect to $P\left(x^{1}, y^{1}\right)$, with $\left(x^{\natural}, y^{1}\right):=\lim _{\beta \uparrow 1}\left(x^{\beta}, y^{\beta}\right)$.

Bewley \& Kohlberg [1976] made a thorough study of asymptotic properties of $v_{\beta}^{\prime}$ and of stationary $\beta$-discounted optimal strategies $x^{\beta}, y^{\beta}$ as $\beta$ tends to 1 . Using Tarski's principle on real closed fields (cf. Tarski [1951]) they derived the following remarkable theorem, which we give without proof.

\subsubsection{THEOREM}

For any zero-sum stochastic gume situation there exist $N \in \mathbb{N},\left\{\alpha_{n} \in \mathbb{R}^{2}: n \in \mathbb{N}_{0}\right\}$, $\left\{x_{n} \in \underset{s=1}{X} \mathbb{R}^{m_{n}}: n \in \mathbb{N}_{0}\right\},\left\{y_{n} \in \dot{X}_{s} \mathbb{R}^{n_{3}}: n \in \mathbb{N}_{0}\right\}$ such that for all $\beta$ close to $I$ :

a) $v_{\beta}^{1}=\sum_{n=0}^{\infty} \alpha_{m}(1-\beta)^{n / N}$ is the $\beta$-discounted value;

b) $x^{\beta}=\sum_{n=0}^{\infty} x_{n}(1-\beta)^{n / N}$ is a stationary $\beta$-discounted optimal strategy for player $1_{s}$

c) $y^{\beta}=\sum_{n=0}^{\infty} y_{n}(1-\beta)^{n / N}$ is a stationary $\beta$-discounted optimal strategy for player 2 .

Two remarks should directly be made about this theorem. First of all it follows that $\lim _{\beta \uparrow 1} v_{\beta}^{1}$ exists and equals $\alpha_{0}$. Second, it follows that $\lim _{\beta \uparrow 1} x^{\beta}$ exists and equals $x_{0}$, which therefore is a stationary strategy.

As an illustration of the ubove theorem observe that for the big match, example 1.7.4, we have $v_{\beta}^{1}=v^{1}$ for all $\beta \in[0,1)$ and for player 1 the unique stationary optimal strategies are given by $x^{\beta}=(1 /(2-\beta),(1-\beta) /(2-\beta))$. Hence we have:

$x^{\beta}=\left[\begin{array}{l}1 \\ 0\end{array}\right]+\left[\begin{array}{c}-1 \\ 1\end{array}\right](1-\beta)+\left[\begin{array}{c}1 \\ -1\end{array}\right](1-\beta)^{2}+\left[\begin{array}{c}-1 \\ 1\end{array}\right](1-\beta)^{3}+\left[\begin{array}{c}1 \\ -1\end{array}\right](1-\beta)^{4}+\ldots$.

For player 2 we have $y^{\beta}=\left[\begin{array}{l}1 / 2 \\ / 2\end{array}\right]$ for all $\beta$.

To give another illustration we examine one more example, where payoffs are again given only for player 1 . 


\subsubsection{EXAMPLE}

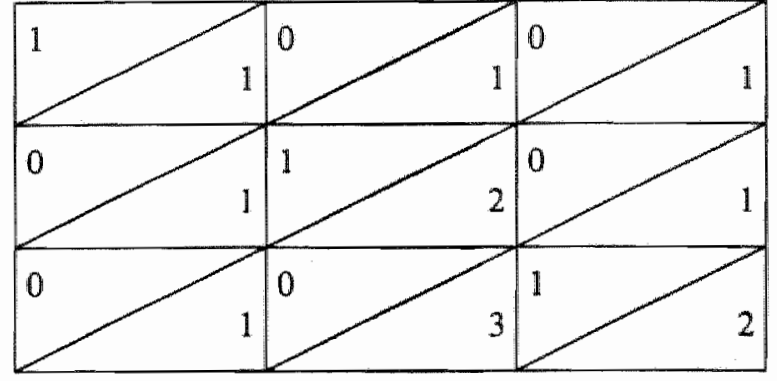

State 1

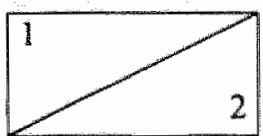

State 2

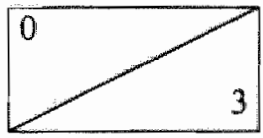

State 3

For this example, solving the Shapley-equation (cf. 1.7.3) leads to the $\beta$ discounted value $v_{\beta}^{1}$ and to the unique stationary optimal strategies $x^{\beta}$ and $y^{\beta}$ given, for initial state 1, by:

$v_{\beta}^{1}=\frac{1-(1-\beta)^{1 / 3}}{\beta}=1-(1-\beta)^{1 / 3}+(1-\beta)-(1-\beta)^{4 / 3}+(1-\beta)^{2}-(1-\beta)^{1 / 3}+\ldots$.

$x^{\beta}=\left[\begin{array}{l}1 \\ 0 \\ 0\end{array}\right]+\left[\begin{array}{c}-1 \\ 1 \\ 0\end{array}\right](1-\beta)^{1 / 3}+\left[\begin{array}{c}0 \\ 1 \\ 1\end{array}\right](1 \quad \beta)^{2 / 3}+\left[\begin{array}{c}1 \\ 0 \\ -1\end{array}\right](1-\beta)+\left[\begin{array}{c}-1 \\ 1 \\ 0\end{array}\right](1-\beta)^{4 / 3}+\left[\begin{array}{c}0 \\ -1 \\ 1\end{array}\right](1-\beta)^{3 / 3}+\ldots .$.

$y^{\beta}=\left[\begin{array}{l}1 \\ 0 \\ 0\end{array}\right]+\left[\begin{array}{c}-1 \\ 0 \\ 1\end{array}\right](1-\beta)^{1 / 3}+\left[\begin{array}{c}0 \\ 1 \\ -1\end{array}\right](1 \quad \beta)^{2 / 3}+\left[\begin{array}{c}1 \\ -1 \\ 0\end{array}\right](1-\beta)+\left[\begin{array}{c}-1 \\ 0 \\ 1\end{array}\right](1-\beta)^{4 / 3}+\left[\begin{array}{c}0 \\ 1 \\ -1\end{array}\right](1-\beta)^{3 / 3}+\ldots$.

The work of Blackwell \& Ferguson [1968] on the big match was generalized by Kohlberg [1974] for zero-sum repeated games with absorbing states (cf. section 4.4). These papers, together with the above result of Bewley \& Kohlberg [1976] for the asymptotic properties of the $\beta$-discounted solutions, were important for the derivation of the following result by Mertens \& Neyman [1981].

\subsubsection{THEOREM}

For any zero-sum stochustic game the limiting average value $v^{\prime \prime}$ exists, and it is related to the $\beta$-discounted values by $v^{1}=\lim _{\beta \uparrow 1} v_{\beta}^{1}$.

Observe that this theorem implies that $v^{1}=\alpha_{0}$, the leading term of the power series, for $\beta$ close to 1 , in theorem 1.7.5. In chapter 5 on stochastic games with respect to the total reward criterion we will see that if the total value $v_{T}^{1}$ exists in $\mathbb{R}^{2}$ and if both players have stationary total optimal strategies, then $\alpha_{0}, \alpha_{1}, \ldots, \alpha_{N-1}$ are all equal to 0 and $\nu_{T}^{1}=\alpha_{N}=\lim _{\beta \uparrow 1}(1-\beta)^{-1} v_{\beta}^{1}$. However, for stochastic games with respect to the total reward criterion little is known and in general the total value $v !$ will not exist. Even on the condition that the limiting average value is 0 (for all starting states), the total value is not necessarily finite. For a further discussion on stochastic games with respect to the total reward criterion we refer to chapter 5, where it is also shown that, like for the 



\subsubsection{THEOREM}

\section{Let $\beta \in[0,1)$.}

a) For any stochastic game there exists a stationary $\beta$-discounted equilibrium.

b) A pair of stationary strategies $\left(x^{*}, y^{*}\right)$ is a $\beta$-discounted equilibrium if for each $s \in S$, the pair of mixed actions $\left(x_{s}^{*}, y_{s}^{*}\right)$ is an equilibrium in the bimatrix game $\left(A_{\beta}^{1 s}\left(\gamma_{\beta}^{1}\left(x^{*}, y^{*}\right)\right), A_{\beta}^{2}\left(\gamma_{\beta}^{2}\left(x^{*}, y^{*}\right)\right)\right)$, where $(c f .1 .7 .3)$ $A_{\beta}^{k s}\left(\gamma_{\beta}^{k}\left(x^{*}, y^{*}\right)\right)=\left[(1-\beta) r^{k}\left(s, i_{j} j\right)+\beta \sum_{i=1}^{z} p(t \mid s, i, j) \gamma\left(t, x^{*}, y^{*}\right)\right]_{i=1, j=1}^{m_{3}}$

Other proofs for this theorem have been given by Takahashi [1964], Rogers [1969] and Sobel [1971].

In the previous section we have seen that for zero-sum stochastic games the existence of the limiting average value and of limiting average e-optimal strategies remained a problem until 1980. The existence of limiting average $\epsilon$ equilibria is even a tougher problem, for it is still open. One should observe that the existence of $\epsilon$-equilibria $\left(\pi_{e}, \sigma_{\varepsilon}\right)$, for all $\epsilon>0$, in a zero-sum stochastic game implies that the value exists and that the strategies $\pi_{\varepsilon}$ and $\sigma_{\varepsilon}$ are $\epsilon$ optimal for the respective players.

If in a general-sum stochastic game an $t$-equilibrium $\left(\pi_{e}, \sigma_{\ell}\right)$ exists, then player 1 would have a reward which is at least the value, possibly up to $c$, of the zero-sum stochastic game obtained by assuming that the payoffs to player 1 have to be payed by player 2 . This is due to the fact that for a $\delta$-optimal strategy $\pi_{\delta}$ for player 1 in that zero-sum game we have that $\gamma^{1}\left(\pi_{\epsilon}, \sigma_{\epsilon}\right) \geqslant \gamma^{1}\left(\pi_{\delta}, \sigma_{\epsilon}\right)-\epsilon>v^{1}-\delta-\epsilon$. Letting $\delta$ tend to 0 gives the result. So we have:

\subsubsection{REMARK}

If $\left(\pi_{\varepsilon}, \sigma_{\varepsilon}\right)$ is a limiting average c-equilibrium, then $\gamma^{k}\left(\pi_{\varepsilon}, \sigma_{\varepsilon}\right) \geqslant v^{k}-c$, where $v^{k}$ is the limiting average value of the zero-sum stochastic game obtained by assuming that the payoffs to player $k$ have to be paid by player $(3-k)$. "the $k$-zeromsum strochastic games. Of course a similar statement holds for the $\beta$-discounted reward case and for the total reward case.

Another interesting fact concerning these $k$-zero-sum stochastic games is that player $(3-k)$ has for each $c>0$ a strategy to keep player $k$ 's reward below $v^{k}+\epsilon$. This allows player $(3-k)$ to punish player $k$ if that is required, and hence player $(3-k)$ can threaten to punish player $k$ if player $k$ deviates from a certain strategy. Punishment arguments to establish equilibria are quite common in the theory of repeated games (cf. Aumann [1981). In the next chapter this will also become meaningful for general stochastic games.

\subsubsection{DEFINITION}

For a general-sum stochastic game $\left(v^{1}, v^{2}\right)$ is called the limiting average threatpoint. A retaliation strategy $\pi_{i}^{r}$ for player $l$ is a strategy for which $\gamma^{2}\left(\pi_{\theta}^{r}, \sigma\right) \leqslant v^{2}+\epsilon$ for all $\sigma$. Similarly we have a retaliation strategy $a_{\varepsilon}^{r}$ for player 2 . 
For zero-sum stochastic games we have $v^{k}=\lim _{\beta_{1} 1} v_{\beta}^{k}$ (cf. 1.7.7). For generalsum stochastic games we can take (by theorem 1.8.3), for each $\beta \in[0,1$ ), a stationary $\beta$-discounted equilibrium $\left(x^{\beta}, y^{\beta}\right)$ and, without loss of generality, we can assume that $\lim _{\beta \uparrow 1}\left(x^{\beta}, y^{\beta}\right)$ and $\lim _{\beta \uparrow 1} \gamma_{\beta}^{k}\left(x^{\beta}{ }_{,} y^{\beta}\right)$ exist and are equal to $\left(x^{i}, y^{1}\right)$ and $V^{k}$ respectively (cf. section 2.2 ). Now one could hope that $V^{k}$ is related with a limiting average e-equilibrium and one could even think that $\left(x^{1}, y^{1}\right)$ may be a limiting average c-equilibrium. Unfortunately this will not be true in general, as is illustrated by the next example which has been examined by Sorin [1986].

\subsubsection{EXAMPLE}

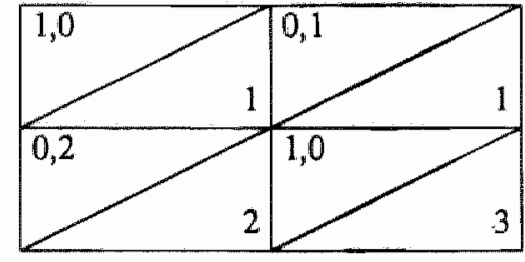

State 1

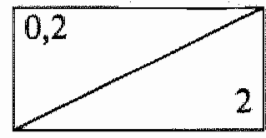

State 2

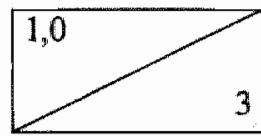

State 3

The 1-zero-sum stochastic game of 1.8 .6 is exactly 1.7 .4 , the big match. The 2-zero-sum stochastic game is ulso a kind of big match.

The unique stationary $\beta$-discounted equilibria $\left(x^{\beta}, y^{\beta}\right)$ for this example are given by (the mixed actions in state 1):

$$
\left(x^{\beta}, y^{\beta}\right)=((2 /(3-\beta),(1-\beta) /(3-\beta)),(1 / 2,1 / 2)) .
$$

So we have $\gamma_{\beta}^{1}\left(1, x^{\beta}, y^{\beta}\right)=1 / 2=v^{1}(1)$ and $\gamma_{\beta}^{2}\left(1, x^{\beta}, y^{\beta}\right)=2 / 3=v^{2}(1)$ for all $\beta \in[0,1)$. Then we find that for $\left(x^{1}, y^{1}\right)=\lim _{\beta \uparrow 1}\left(x^{\beta}, y^{\beta}\right)=((1,0),(1 / 2,1 / 2))$ the limiting average rewards are:

$$
\gamma^{1}\left(1, x^{1}, y^{1}\right)=1 / 2=v^{1}(1) \text { and } \gamma^{2}\left(1, x^{1}, y^{1}\right)=1 / 2<3 / 3=v^{2}(1) \text {. }
$$

It is obvious that $\left(x^{1}, y^{1}\right)$ is no limiting average e-equilibrium, because against $x^{1}$ player 2 could improve by playing $(0,1)$. Sorin [1986] shows that for this example the limiting average rewards corresponding with limiting average $\mathrm{\epsilon}$ equilibria for state 1 are all in the convex hull $L$ of $\{(1 / 2,1),(2 / 3,2 / 3)\}$. So $L=\{(a, 2-2 a): a \in[1 / 2,2 / 3]\}$.

It is important to observe that for $x^{*}=(0,1)$ we find:

$$
\begin{aligned}
& \gamma^{1}\left(1, x^{\beta}, y^{1}\right)=\gamma^{1}\left(1, x^{*}, y^{1}\right)=1 / 2=v^{1}(1) \text { and } \\
& \gamma^{2}\left(1, x^{\beta}, y^{1}\right)=\gamma^{2}\left(1, x^{*}, y^{1}\right)=1>2 / 3=v^{2}(1) .
\end{aligned}
$$

The importance of this observation will become clear in chapter 3 (cf. example 3.2.4).

In graph: 


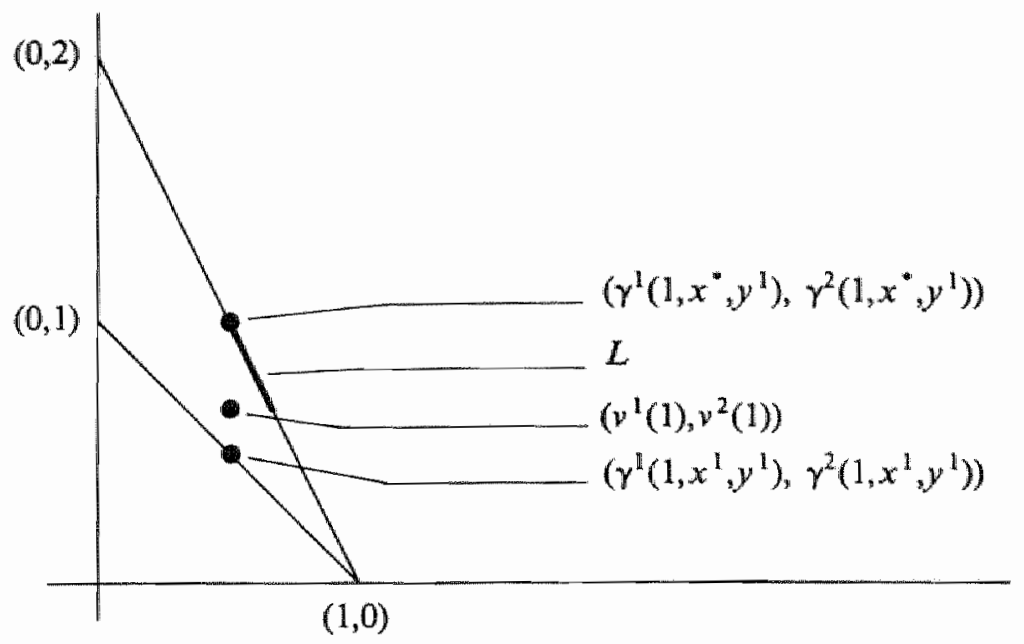

In this graph the area in the triangle $((1,0),(0,1),(0,2))$ is the set of feasible rewards for this stochastic game, i.e. those rewards that can occur for some pair of strategies. The feasible rewards which are larger than the threat-point are called individually rational. The set $L$ consists of the feasible, individually rational Pareto optimal rewards, where the Pareto optimal rewards are those rewards that cannot be improved simultaneously for both players.

Although the above example suggests a gap between general-sum $\beta$ discounted solutions and limiting average solutions, we exhibit in the next chapters that limiting average $\epsilon$-equilibria may, under some condition, be derived from sequences of stationary $\beta$-discounted equilibria. 



\section{Chapter 2 \\ Particular initial states in stochastic games}

\subsection{INTRODUCTION}

In chapter 1 we have seen that for any zero-sum stochastic game the $\beta$ discounted value exists and that both players have stationary $\beta$-discounted optimal strategies; in the general-sum case there exist stationary $\beta$-discounted equilibria. We have also seen in chapter 1 that for any zero-sum stochastic game the limiting average value exists, which however does not guarantee the existence of optimal strategies or stationary c-optimal strategies; in the general-sum case the existence of limiting average c-equilibria is still an open problem. As discussed in chapter 1, for the limiting average reward criterion more complex strategies are required to play e-optimal or to form an cequilibrium. There may be however, starting states for which a solution exists in terms of stationary strategies. Consider for instance the initial states 2 and 3 of the big match (example 1.7.4). This idea, of examining particular starting states, first occurred in Tijs \& Vrieze [1986]. They showed that for each player there are, in a zero-sum stochastic game, 'easy initial states', i.e. starting states for which this player has a stationary limiting average optimal strategy. Their proof however is rather technical. In this chapter we give an alternative and straightforward proof for their theorem in section 2.4. There we also examine other initial states where both players can achieve $\epsilon$-optimality, with respect to the limiting average reward criterion, by using stationary strategies.

In section 2.3 we examine special initial states in general-sum stochastic games, and we show that for certain 'strong initial states' limiting average cequilibria exist which consist of 'almost stationary strategies". The latter are stationary strategies which are amplified with some threat to prevent profitable deviations of the opponent. So if both players stick to their e-equilibrium strategies, then with probability close to 1 they will use stationary strategies throughout the whole game.

In section 2.2 we derive some basic results, which are of fundamental importance for the chapters 3 and 4 as well. Our techniques are based on properties of sequences of stationary strategies $\left\{\left(x^{\beta}, y^{\beta}\right): \beta \in[0,1)\right\}$, converging for $\beta$ tending to 1 .

The results of this chapter have been derived from Thuijsman \& Vrieze [1989-a, 1989-b]. 


\subsection{LIMIT PROPERTIES FOR SEQUENCES OF STRATEGY PAIRS}

By the expression

'let $\left\{\left(x^{\beta}, y^{\beta}\right) \in X \times Y: \beta \in[0,1)\right\}$ be a (conwerging) sequence with $\lim _{\beta \uparrow !}\left(x^{\beta}, y^{\beta}\right)=\left(x^{1}, y^{11}\right)^{\prime}$

we mean:

'for some sequence $\left\{\beta_{n} \in[0,1): n \in \mathbb{N}\right\}$, with $\lim _{n \rightarrow \infty} \beta_{n}=1$, it holds that $\lim _{n \rightarrow \infty}\left(x^{\beta_{n}}, y^{\beta_{n}}\right)=\left(x^{1}, y^{1}\right) \in X \times Y$.

Observe that $X \times Y$ is compact, which implies that any sequence in $X \times Y$ has a converging subsequence.

\subsubsection{DEFINITION}

Let $\left\{\left(x^{\beta}, y^{\beta}\right) \in X \times Y: \beta \in[0,1)\right\}$ be a sequence with $\lim _{\beta * 1}\left(x^{\beta}, y^{\beta}\right)=$ $\left(x^{1}, y^{1}\right) \in X \times Y$. Without lass of generality we may assume that $\operatorname{Car}^{2}\left(x^{\beta}\right)$ and $\operatorname{Car}^{z}\left(y^{\beta}\right)$ are independent of $\beta<1$. By compactness arguments we can also assume that the following limits exist and we can define:

a) $V^{k}:=\lim _{\beta \uparrow 1} \gamma_{\beta}^{k}\left(x^{\beta}, y^{\beta}\right) \in[-M, M]^{z}$, for $k=1,2$.

b) $Z^{1}:=\lim _{\beta \uparrow 1} Z^{\beta}$, where $Z^{\beta}:=(1-\beta)\left(I-\beta P\left(x^{\beta}, y^{\beta}\right)\right)^{-1}$.

c) $T$ is the set of states which are transient with respect to $\left(x^{1}, y^{1}\right)$.

$S^{1}, S^{2}, \ldots, S^{H}$ are the ergodic sets with respect to $\left(x^{1}, y^{1}\right)$.

For each $h \in\{1,2, \ldots, H\}$ let $\tilde{q}^{h} \in \Delta^{\left|s^{k}\right|}$ be the unique stationary distribution of $P\left(x^{1}, y^{l}\right)^{h}$, the restriction of $P\left(x^{1}, y^{1}\right)$ to $S^{h}$; let $q^{h} \in \Delta^{z}$ be the related stationary distribution (for initial states in $S^{h}$ ) of $P\left(x^{1}, y^{1}\right)$ on $S$, i.e. $q_{s}^{h}=\tilde{q}_{s}^{h}$ for $s \in S^{h}$ and $q_{s}^{h}=0$ for $s \notin S^{h}$.

Observe that for all $\beta \in[0,1)$ all row sums of $Z^{\beta}$ are equal to 1 and all entries of $Z^{\beta}$ are non-negative. Hence $Z^{\beta}, \beta \in[0,1)$, and $Z^{1}$ are stochastic matrices.

In this section $\left\{\left(x^{\beta}, y^{\beta}\right): \beta \in[0,1)\right\}$ is a sequence as in definition 2.2.1.

\subsubsection{REMARK}

Let $Q\left(x^{1}, y^{1}\right)^{h}$ denote the restriction of $Q\left(x^{1}, y^{1}\right)$ to $S^{h}$ and let $s \in S^{h}$. Then the $s-t h$ row of $Q\left(x^{1}, y^{1}\right)^{\text {h }}$ equals $\tilde{q}^{h}$ and is strictly pasitive. Furthermore the $s$-th row of $Q\left(x^{1}, y^{1}\right)$ equals $q^{h}$.

By ordening the states, the matrices $P\left(x^{1}, y^{1}\right)$ and $Q\left(x^{1}, y^{1}\right)$ will have the following shape:

$$
P\left(x^{1}, y^{1}\right)=\left[\begin{array}{ccccc}
P\left(x^{1}, y^{1}\right)^{1} & 0 & \ldots & 0 & 0 \\
0 & P\left(x^{1}, y^{1}\right)^{2} & & & \\
& & & 0 & \\
0 & 0 & & P\left(x^{1}, y^{1}\right)^{H} & 0 \\
P\left(x^{1}, y^{1}\right)^{T 1} & P\left(x^{1}, x^{1}\right)^{T 2} & \ldots & P\left(x^{1}, y^{1}\right)^{T H} & P\left(x^{1}, y^{1}\right)^{T}
\end{array}\right]
$$




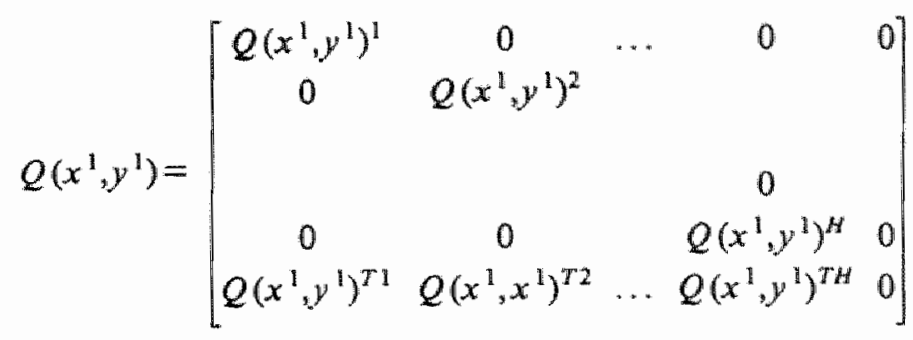

Here $P\left(x^{1}, y^{1}\right)^{T h}$ and $Q\left(x^{1}, y^{1}\right)^{T h}$ are restrictions of $P\left(x^{1}, y^{1}\right)$, resp. $Q\left(x^{1}, y^{1}\right)$, to rows in $T$ and columns in $S^{h}$; similarly $P\left(x^{1}, y^{1}\right)^{T}$ is the restriction of $P\left(x^{1}, y^{1}\right)$ to rows and columns in $T$.

It is well-known (cf. Kemeny \& Snell [1960]) that for $h \in\{1,2, \ldots, H\}$ :

$$
Q\left(x^{1}, y^{1}\right)^{T_{h}}=\left(I^{T}-P\left(x^{1}, y^{1}\right)^{T}\right)^{-1} P\left(x^{1}, y^{1}\right)^{T h} Q\left(x^{1}, y^{1}\right)^{h} \text {. }
$$

Observe that $\left(I^{T}-P\left(x^{1}, y^{1}\right)^{T}\right)^{-1} P\left(x^{1}, y^{1}\right)^{\text {Th }}$ has $|T|$ rows and $\left|S^{h}\right|$ columns. For $s \in T$ and $t \in S^{\text {th }}$ entry $(s, t)$ of $\left(I^{T}-P\left(x^{1}, y^{1}\right)^{T}\right)^{-1} P\left(x^{1}, y^{1}\right)^{T_{t}}$ gives the probability that a stochastic process which starts in $s$ will ever enter the ergodic set $S^{h}$ through state $t$.

\subsubsection{LEMMA}

a) $\gamma^{k}\left(s, x^{1}, y^{1}\right)=\gamma^{k}\left(t, x^{1}, y^{1}\right)=: \gamma^{k h}\left(x^{1}, y^{1}\right)$ for $s, t \in S^{h}, h \in\{1,2, \ldots, H\}$ and for $k=1,2$.

b) $V^{k}=P\left(x^{1}, y^{1}\right) V^{k}$ for $k=1,2$.

c) $V_{s}^{k}=V_{1}^{k}=: V^{k h}$ for $s, t \in S^{h}, h \in\{1,2, \ldots, H\}$ and for $k=1,2$.

\section{PRoOF:}

By lemma 1.5 .5 it holds that $\gamma^{k}\left(s, x^{1}, y^{1}\right)=Q\left(x^{1}, y^{1}\right)_{s} r^{k}\left(x^{1}, y^{1}\right)$. Now (a) follows from remark 2.2.2.

(b) follows from $\gamma_{\beta}^{k}\left(x^{\beta}, y^{\beta}\right)=(1-\beta) r^{k}\left(x^{\beta}, y^{\beta}\right)+\beta P\left(x^{\beta}, y^{\beta}\right) \gamma_{\beta}^{k}\left(x^{\beta}, y^{\beta}\right)$ (cf. lemma 1.5 .3 (c)). Taking limits and applying definition 2.2 .1 gives the result. Now (b) implies that $V^{k}=Q\left(x^{1}, y^{1}\right) V^{k}$, which by remark 2.2 .2 gives (c).

Although its proof is rather simple, the next lemma turns out to be of great importance in the sequel.

\subsubsection{LEMMA}

$Z^{1} P\left(x^{1}, y^{1}\right)=Z^{1}$.

\section{Proor:}

By definition 2.2 .1 we have $Z^{\beta}\left(I-\beta P\left(x^{\beta}, y^{\beta}\right)\right)=(1-\beta) I$ for all $\beta \in[0,1)$.

Taking limits for $\beta$ going to 1 completes the proof.

The strength of this lemma becomes clear in the lemmas below. 


\subsubsection{LEMMA}

Let $s \in S$ and let $Z_{s}^{1}$ be the $s$ th row of $Z^{1}$.

a) There exists $\mu_{s}=\left(\mu_{s}^{1}, \mu_{s}^{2}, \ldots, \mu_{s}^{H}\right) \in \Delta^{H}$ such that $Z_{s}^{1}=\sum_{h=1}^{H} \mu_{s}^{h} q^{h}$.

b) If for the Markov chain related with $P\left(x^{\beta}, y^{\beta}\right)$ the probability of ever reaching $S^{h}$ is 0 when starting in $s$, then $\mu_{s}^{h}=0$.

Proof:

a) By lemma 2.2 .4 it holds that $Z_{s}^{1}$ is a stationary distribution of the Markov chain related with $P\left(x^{1}, y^{1}\right)$. Since the set of all stationary distributions for $P\left(x^{1}, y^{1}\right)$ is the convex hull of $\left\{q^{1}, q^{2}, \ldots, q^{H}\right\}$, there is $\mu_{s} \in \Delta^{H}$ as desired.

b) If under $\left(x^{\beta}, y^{\beta}\right)$ the set $S^{h}$ cannot be reached when starting in $s$, then it follows that entry $(s, t)$ of $P^{n}\left(x^{\beta}, y^{\beta}\right)$ is 0 for all $n \in \mathbb{N}$ and all $t \in S^{h}$.

Hence for all $t \in S^{h}$ we have that entry $(s, t)$ of

$Z^{\beta}=(1-\beta) \sum_{n=1}^{\infty} \beta^{n-1} P^{n \cdot 1}\left(x^{\beta}, y^{\beta}\right)$ is 0 . But then entry $(s, t)$ of $Z^{1}$ is also

0 for all $t \in S^{h}$, which implies that $\mu_{s}^{h}=0$.

The next lemma says: 'the limit of discounted rewards equals a convex combination of the limiting average rewards for the limit strategies.'

\subsubsection{LEMMA}

Let $s \in S$ and let $\mu_{s} \in \Delta^{H}$ be as in lemma 2.2.5.

Then $\mathbb{V}_{s}^{k}=\sum_{h=1}^{H} \mu_{s}^{h} \gamma^{k h}\left(x^{1}, y^{1}\right)$ for $k=1,2$.

\section{Proof:}

Let $<,>$ denote the inner product.

By definition 2.2.1, remark 2.2.2 and lemmas 2.2.3 and 2.2.5 we have:

$$
\begin{aligned}
V_{s}^{k} & =\lim _{\beta \uparrow 1} \gamma_{\beta}^{k}\left(s, x^{\beta}, y^{\beta}\right)=\lim _{\beta \uparrow 1}\left\langle Z_{s}^{\beta}, r^{k}\left(x^{\beta}, y^{\beta}\right)\right\rangle=\left\langle Z_{s}^{1}, r^{k}\left(x^{1}, y^{1}\right)\right\rangle \\
& =\sum_{h=1}^{H} \mu_{s}^{h}\left\langle q^{h}, r^{k}\left(x^{1}, y^{1}\right)\right\rangle=\sum_{h=1}^{H} \mu_{s}^{h} \gamma^{k h}\left(x^{1}, y^{1}\right) .
\end{aligned}
$$

\subsubsection{COROLLARY}

There exist $h^{1}{ }^{1} h^{2} \in\{1,2, \ldots, H\}$ such that:

$\gamma^{l h^{\prime}}\left(x^{1}, y^{1}\right) \geqslant \max _{s \in S} V_{s}^{1}$ and $\gamma^{2 h^{2}}\left(x^{1}, y^{1}\right) \geqslant \max _{s \in S} V_{s}^{2}$.

\subsection{STRONG INITIAL STATES IN THE GENERAL-SUM CASE}

We show that, in any stochastic game, there are some starting states for which there exists an almost stationary limiting average $\epsilon$-equilibrium. 


\subsubsection{DEFINITION}

An almost stationary limiting average c-equilibrium for initial state $s$ is a pair of strategies $\left(\pi^{*}, \sigma^{*}\right)$ such that $\left(\pi^{*}, \sigma^{*}\right)$ is a limiting average cequilibrium for initial state $s$ and $\left(\pi^{*}, \sigma^{*}\right)$ consists of stationary strategies $\left(x^{*}, y^{*}\right)$ and retaliation strategies $\left(\pi^{\prime \prime}, \sigma^{\prime}\right)$. Player 1 uses $x^{*}$ unless he detects a deviation of player 2 from $\sigma^{*}$, in which case player 1 immediately turns to using $\pi^{*}$. For player $2 \sigma^{*}$ is a similar strategy.

A strong initial state is an initial state for which there exists an almost stationary limiting average $\epsilon$-equilibrium, for all $\mathrm{\epsilon}>0$.

Since we are dealing with strategies where mixed actions can be used at all stages, the phrase "unless player 2 deviates from $\sigma^{*}$ " should be interpreted as: 'unless player 1 knows that the probability of player 2 playing $a$ " is close to 0. .'

\subsubsection{REMARK}

In this section let $\left\{\left(x^{\beta}, y^{\beta}\right): \beta \in[0,1)\right\}$ be a sequence of stationary $\beta$-discounted equilibria with $\lim _{\beta \uparrow 1}\left(x^{\beta}, y^{\beta}\right)=\left(x^{1}, y^{1}\right)$ and which furthermore suits definition 2.2.1.

In addition to the results developed in the previous section for such a sequence $\left\{\left(x^{\beta}, y^{\beta}\right): \beta \in[0,1)\right\}$, the fact that we are dealing with stationary $\beta$-discounted equilibria allows us to conclude the following.

\subsubsection{LEMMA}

a) For each $\tilde{x} \in X$ with $\operatorname{Car}^{2}(\tilde{x}) \subset \operatorname{Car}^{z}\left(x_{\beta}^{1}\right)$ and for all $x \in X$ :

$$
V^{1}=P\left(x^{1}, y^{1}\right) V^{1}=P\left(\tilde{x}, y^{1}\right) V^{1} \geqslant P\left(x, y^{1}\right) V^{1} \text {. }
$$

b) For each $\tilde{y} \in Y$ with $\operatorname{Car}^{2}(\tilde{y}) \subset \operatorname{Car}^{z}\left(y_{\beta}^{1}\right)$ and for all $y \in Y$ :

$$
V^{2}=P\left(x^{1}, y^{1}\right) V^{2}=P\left(x^{1}, \tilde{y}\right) V^{2} \geqslant P\left(x^{1}, y\right) V^{2} \text {. }
$$

c) $V^{k} \geqslant v^{k}$ for $k=1,2$.

\section{Proof:}

By lemma 1.6.4 we have for all $\beta \in[0,1)$ :

$$
\begin{aligned}
\gamma_{\beta}^{1}\left(x^{\beta}, y^{\beta}\right) & =(1-\beta) r^{1}\left(x^{\beta}, y^{\beta}\right)+\beta P\left(x^{\beta}, y^{\beta}\right) \gamma_{\beta}^{1}\left(x^{\beta}, y^{\beta}\right) \\
& =(1-\beta) r^{1}\left(\tilde{x}, y^{\beta}\right)+\beta P\left(\tilde{x}, y^{\beta}\right) \gamma_{\beta}^{1}\left(x^{\beta}, y^{\beta}\right) \\
& \geq(1-\beta) r^{1}\left(x, y^{\beta}\right)+\beta P\left(x, y^{\beta}\right) \gamma_{\beta}^{1}\left(x^{\beta}, y^{\beta}\right) .
\end{aligned}
$$

Taking limits for $\beta$ to 1 proves (a). The proof of $(b)$ is similar. By remark 1.8.4 we have $\gamma_{\beta}^{k}\left(x^{\beta}, y^{\beta}\right) \geq v_{\beta}^{k}$ for all $\beta \in[0,1)$, hence (c) follows by taking limits (cf. definition 2.2.1 and theorem 1.7.7).

Lemma 2.3 .3 implies that, if $\gamma^{k}\left(x^{1}, y^{1}\right) \geqslant V^{k}$ for both players and if each of them can check whether or not his opponent is actually using $y^{1}$ or $x^{1}$, then one could construct a limiting average $\epsilon$-equilibrium. This is possible by using 
a threat to retaliate, giving less than $v^{k}+\epsilon$ in case of a detected deviation. This is worked out more precisely in the next lemma.

\subsubsection{LEMMA}

If for $h \in\{1,2, \ldots, H\}$ it holds that $\gamma^{k h}\left(x^{1}, y^{1}\right) \geqslant V^{k h}$ for $k=1$ as well as for $k=2$, then a limiting average c-equilibrium for initial states in $S^{h}$ can be made by supplementing $x^{1}$ and $y^{1}$ with suitable retaliation threats.

\section{Proof:}

Let $\epsilon>0$ and let $h \in\{1,2, \ldots, H\}$ be such that $\gamma^{k h}\left(x^{1}, y^{1}\right) \geqslant V^{k h}$ for $k=1$ and 2 . We divide the proof in three parts: in part 1 we show that each player can detect deviations of his opponent with probability close to 1 ; in part 2 we show that each player can retaliate if he detects a deviation; in part 3 we show that $\left(x^{\|}, y^{1}\right)$ supplemented with retaliation threats is a limiting average $c$ equilibrium on $S^{n}$.

PART 1: Player I can detect deviations of player 2 with probability clase to 1. Suppose player 1 uses $x^{1}$.

It is clear that if player 2 at some stage chooses an action outside $\operatorname{Car}^{2}\left(y^{11}\right)$, then player 1 immediately knows that player 2 is not using $y^{\sharp}$.

As long as player 2 chooses actions within $\operatorname{Car}^{2}\left(y^{1}\right)$, the play will remain within $S^{h}$ and player 1 can count the number of times that player 2 chooses action $j$ in state $s \in S^{h}$ for all $j$ and $s$. Hence at each stage $n \in \mathbb{N}$ player 1 knows the action frequency $y_{s}^{(n)}(j)$ of action $j$ in state $s$. If player 2 really uses $y^{1}$, then $y_{s}^{(n)}(j)$ should converge to $y_{s}^{y}(j)$ as $n$ goes to infinity.

Let $Y_{s}^{(n)}(j)$ be the random variable which denotes the action frequency of action $j$ in state $s$. So $y_{s}^{(n)}(j)$ is a realization of $Y_{s}^{(n)}(j)$. It is well-known (cf. Billingsley [1979]) that for every $\alpha, \delta>0$ there exists $N_{\alpha \delta} \in \mathbb{N}$ such that:

$$
\text { Prob }_{x^{\prime}, y^{\prime}}\left\{\left\|Y_{s}^{(n)}-y_{s}^{1}\right\|>\alpha \text { for some } s \in S^{h} \text { and some } n \geqslant N_{\alpha \delta}\right\}<\delta \text {. }
$$

If for all $n \geqslant N_{\alpha \delta}$ and all $s \in S^{h}$ it holds that $\left\|y_{s}^{(n)}-y_{s}^{1}\right\| \leqslant \alpha$ then, by continuity arguments, the limiting average reward to player $k$ is at most $\gamma^{k h}\left(x^{1}, y^{1}\right)+\alpha K$ and at least $\gamma^{k h}\left(x^{1}, y^{1}\right)-\alpha K$ for some constant $K \in \mathbb{N}$. So if player 1 does not detect a deviation of player 2 , then the limiting average reward to player 2 is at most $\gamma^{2 / h}\left(x^{1}, y^{1}\right)+\alpha K$.

PART 2: Player 1 can retaliate if he detects a deviation of player 2.

Suppose at some stage $n$ player 1 detects a deviation of player 2, i.e. player 2 chooses $f$ at that stage in state $s$ and either $j \notin \operatorname{Car}\left(y_{s}^{\prime}\right)$ or $n \geqslant N_{\alpha \delta}$ and $\left\|y_{s}^{(n)}-y_{s}^{3}\right\|>\alpha_{w}$

If, from stage $n+1$ on, player 1 now uses a retaliation strategy $\pi_{\varepsilon / 2}^{r}$ (cf. definition 1.8.5) then the limiting average reward to player 2 will be at most $\sum_{i=1}^{\sum} p\left(t \mid s, x_{s}^{1}, j\right)\left(v_{t}^{2}+\epsilon / 2\right)$.

By lemma 2.3 .3 we have: 


$$
\sum_{i=1}^{z} p\left(t \mid s, x_{s}^{1}, j\right)\left(v_{t}^{2}+c / 2\right) \leqslant \sum_{i=1}^{z} p\left(t \mid s, x_{s}^{1}, j\right)\left(V_{t}^{2}+\epsilon / 2\right) \leqslant V_{s}^{2}+\epsilon / 2 .
$$

Since $\gamma^{2 h}\left(x^{1}, y^{1}\right) \geqslant V^{2 h}=V_{s}^{2}$ (cf. lemma 2.2.3), we conclude that the limiting average reward to player 2 will be at mos: $\gamma^{2 h}\left(x^{1}, y^{1}\right)+c / 2$.

PART 3: $\left(x^{1}, y^{1}\right)$ can be supplemented with retaliation threats to become a limiting average e-equilibrium for all starting states in $S^{h}$.

Let $\alpha \in(0, \epsilon / 4 K)$ and take $\delta>0$ such that

$(1-\delta)^{2}\left(\gamma^{k h}\left(x^{1}, y^{1}\right)-\alpha K\right)-\left(1-(1-\delta)^{2}\right) M \geqslant \gamma^{k h}\left(x^{1}, y^{1}\right)-\epsilon / 2$ for $k=1,2$.

Now player 1 can try to keep player 2 from deviating from $y^{1}$ by using the almost stationary strategy $\pi_{\varepsilon}^{*}$ defined by:

a) use $x^{1}$ unless:

i) player 2 chooses an action outside $\operatorname{Car}^{2}\left(y^{1}\right)$, or

ii) for some $n \geq N_{\alpha \delta}$ and some $s \in S^{h}:\left\|y_{s}^{(m)}-y_{s}^{1}\right\|>\alpha$.

b) if (i) or (ii) occurs, start retaliation by using $\pi_{\varepsilon / 2}^{r}$ from that stage on.

For player 2 the almost stationary strategy $\sigma_{e}^{*}$ is defined analogously.

From these definitions it follows that:

$\gamma^{k h}\left(\pi_{e}^{*}, \sigma_{e}^{*}\right) \geqslant(1-\delta)^{2}\left(\gamma^{k h}\left(x^{1}, y^{1}\right)-\alpha K\right)-\left(1-(1-\delta)^{2}\right) M \geqslant \gamma^{k h}\left(x^{1}, y^{1}\right)-\epsilon / 2$.

From parts 1 and 2 we conclude that for all $\sigma \in \Sigma$ :

$$
\gamma^{2 h}\left(\pi^{*}, \sigma\right) \leqslant \gamma^{2 h}\left(x^{1}, y^{1}\right)+\epsilon / 2
$$

Similarly one can derive that for all $\pi \in \Pi$ :

$$
\gamma^{1 h}\left(\pi, \sigma_{e}^{*}\right) \leqslant \gamma^{l h}\left(x^{1}, y^{1}\right)+\epsilon / 2 \text {. }
$$

Hence $\left(\pi_{\varepsilon}^{*}, \sigma_{\varepsilon}^{*}\right)$ is an almost stationary limiting average e-equilibrium for all starting states in $S^{h}$.

\subsubsection{THEOREM}

For any general-sum stochastic game there exist strong initial states.

It is clear that this theorem follows directly from lemma 2.3 .6 below which states that the condition of lemma 2.3.4 is automatically fulfilled for some $h$.

\subsubsection{LEMMA}

There exists $h^{*} \in\{1,2, \ldots, H\}$ such that:

$$
\gamma^{1 h^{\circ}}\left(x^{1}, y^{1}\right) \geqslant \max _{h} V^{1 h} \geqslant V^{1 h^{\circ}} \text { and } \gamma^{2 h^{\circ}}\left(x^{1}, y^{1}\right) \geqslant V^{2 h^{\circ}} .
$$

Proof:

For a non-empty $E \subset\{1,2, \ldots, H\}$ let $S^{E}:=\bigcup_{h \in E} S^{h}$.

Let $E_{1}:=\left\{h \in\{1,2, \ldots, H\}: \gamma^{\text {th }}\left(x^{1}, y^{1}\right) \geq \max _{s} V_{s}^{1}\right\}$. Then by corollary 2.2 .7 we have that $E_{1} \neq \varnothing$. 
Now let $T_{1}$ be the set of transient states for which plays started there lead to $S^{E_{1}}$ under $\left(x^{1}, y^{1}\right)$ with probability 1 . So $T_{1}:=\left\{s \in T\right.$ : entry $(s, t)$ of $Q\left(x^{1}, y^{1}\right)$ is 0 for all $\left.t \notin S^{E_{i}}\right\}$.

Now take a stationary strategy $x^{*}$ for player 1 such that for $\beta \in[0,1)$ :

$x_{s}^{*}=x_{s}^{\|}$for all $s \notin S^{E} \cup T_{1}$ and $\operatorname{Car}\left(x_{s}^{*}\right)=\operatorname{Car}\left(x_{s}^{\beta}\right)$ for all $s \in S^{E_{1}} \cup T_{1}$. Remember that $\operatorname{Car}\left(x_{s}^{\beta}\right)$ is independent of $\beta \in[0,1)$ (cf. definition 2.2.1). Observe that each ergodic set with respect to $\left(x^{*}, y^{1}\right)$ is either one of the sets $S^{h}$ with $h \notin E_{1}$ or it is a subset of $T_{1} \cup S^{E_{1}}$.

In order to prove that for some $h^{*} \in E_{1}$ it holds that $\gamma^{2 h^{\circ}}\left(x^{1}, y^{1}\right) \geqslant V^{2 h^{*}}$ we make use of the following observation. There are $E_{2}$ and $T_{2}$ with $\varnothing \neq E_{2} \subset E_{1}$ and $T_{2} \subset T_{1}$ such that $T_{2} \cup S^{E_{2}}$ is an ergodic set with respect to $\left(x^{*}, y^{1}\right)$.

To show the correctness of this statement, suppose that it is not true. Then the ergodic sets with respect to $\left(x^{*}, y^{1}\right)$ are necessarily the sets $S^{h}$ with $h \notin E_{1}$, and $\varnothing \neq(1,2, \ldots, H\} \backslash E_{1}$. But then, using lemma 1.6.4, lemma 2.2 .3 and analogues of lemmas 2.2 .5 and 2.2.6 we conclude that for each $s \in S$ there is $\mu_{s} \in \Delta^{H}$ such that:

$$
\begin{aligned}
V_{s}^{\mathbb{1}} & =\lim _{\beta \uparrow 1} \gamma_{\beta}^{1}\left(s, x^{\beta}, y^{\beta}\right)=\lim _{\beta \uparrow \mathbb{1}} \gamma_{\beta}^{\mathbb{1}}\left(s, x^{*}, y^{\beta}\right)=\sum_{h \notin E_{3}} \mu_{s}^{h} \gamma^{1 h}\left(x^{*}, y^{\mathbb{1}}\right) \\
& =\sum_{h \notin E_{1}} \mu_{s}^{h} \gamma^{1 h}\left(x^{1}, y^{\mathbb{1}}\right)<\max _{t \in S} V_{r}^{1} .
\end{aligned}
$$

Since this is clearly a contradiction, our statement is correct.

So there are $\varnothing \neq E_{2} \subset E_{1}$ and $T_{2} \subset T_{1}$ as desired. By definition of $x^{*}$ and by the fact that $\operatorname{Car}^{z}\left(x^{\beta}\right)$ is independent of $\beta \in[0,1)$ it follows that $T_{2} \cup S^{E_{2}}$ is an ergodic set with respect to $\left(x^{b}, y^{1}\right)$ for all $\beta \in[0,1)$. Once more applying lemma 1.6.4, lemma 2.2 .3 and analogues of lemmas 2.2 .5 and 2.2.6 we obtain that for each $s \in S^{E_{2}}$ there is $\mu_{s} \in \Delta^{H}$ such that:

$$
V_{s}^{2}=\lim _{\beta \uparrow 1} \gamma_{\beta}^{2}\left(s, x^{\beta}, y^{\beta}\right)=\lim _{\beta \uparrow 1} \gamma_{\beta}^{2}\left(s, x^{\beta}, y^{1}\right)=\sum_{h \in E_{2}} \mu_{s}^{h} \gamma^{2 h}\left(x^{1}, y^{1}\right) \leqslant \max _{h \in E_{z}} \gamma^{2 h}\left(x^{1}, y^{1}\right) \text {. }
$$

Hence there is $h^{*} \in E_{2}$ and $s \in S^{h^{*}}$ such that $\gamma^{2 h}\left(x^{1}, y^{1}\right) \geqslant V_{s}^{2}=V^{2 h}$.

\subsubsection{REMARK}

Observe that for the almost stationary limiting average c-equilibria, as constructed for some $S^{h}$ in the proof of lemma 2.3.4, the property holds that a play started in $S^{h}$ will remain in $S^{h}$ with probability close to 1. 


\subsubsection{EXAMPLE}

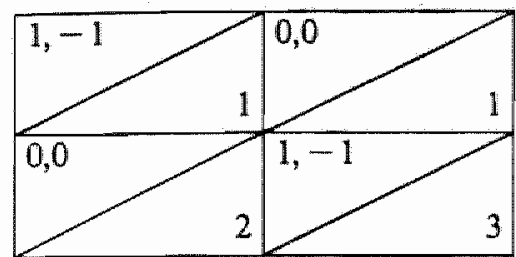

State 1

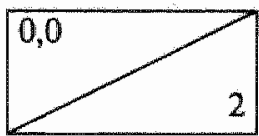

State 2

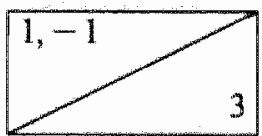

State 3

Notice that this is again the big match (cf. example 1.7.4). For this stochastic game the unique stationary $\beta$-discounted equilibria are given by $\left(x^{\beta}, y^{\beta}\right)=((\mathbb{1} /(2-\beta),(1-\beta) /(2-\beta)),(1 / 2,1 / 2))$ (for starting state 1$)$. It is clear that $\left(x^{1}, y^{1}\right)=((1,0),(1 / 2,1 / 2))$ is not a limiting average $\epsilon$-equilibrium for starting state 1. However, $\gamma^{1}\left(1, x^{1}, y^{1}\right)=1 / 2=V_{1}^{1}=v_{1}^{1}$ and $\gamma^{2}\left(1, x^{1}, y^{1}\right)=-1 / 2=$ $V_{1}^{2}=v_{1}^{2}$. So by lemma 2.3.4 the pair of strategies $\left(x^{1}, y^{1}\right)$ can be supplemented with retaliation threats to become a limiting average $\epsilon$-equilibrium (player 1 has to check whether or not player 2's action-frequencies for state 1 are close to $(1 / 2,1 / 2)$ in the long run; player 1 cannot gain by deviating against $\left.y^{11}\right)$.

So state 1 is a strong initial state for this stochastic game. It is also clear that state 2 and state 3 are strong initial states. Hence we have an almost stationary limiting average t-equilibrium for this stochastic game. Moreover for some starting states ( 2 and 3 ) we even have a stationary limiting average equilibrium. The next example shows that in general there need not be initial states for which there is a stationary limiting average equilibrium.

\subsubsection{EXAMPLE}

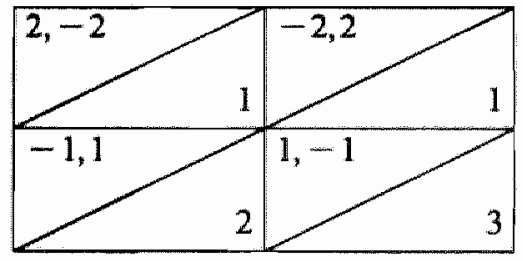

State 1

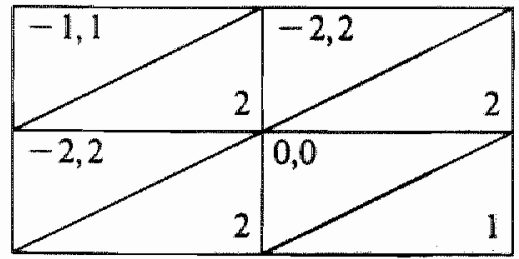

State 2

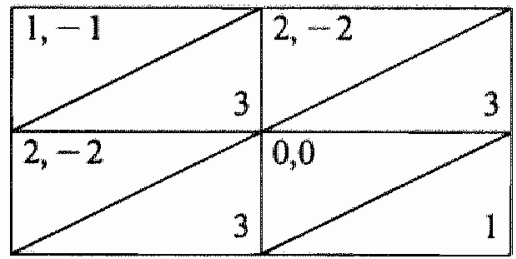

State 3 
For this stochastic game $v^{1}=(0,-1,1)$ and $v^{2}=(0,1,-1)$. Since this stochastic game is in fact a zero-sum stochastic game, any limiting average reward for an equilibrium for initial state $s$ should be equal to the threat-point $\left(v_{s}^{1}, v_{s}^{2}\right)$.

For this stochastic game there is no stationary limiting average equilibrium for any of the initial states. We discuss the initial states one by one.

a) Suppose that $(x, y)$ is a stationary limiting average equilibrium for initial state 1. If $x_{1}=(1,0)$ then $y_{1}=(0,1)$ since $y$ is a best reply against $x$ for player 2. But then $\gamma^{1}(1, x, y)=-2<v 1$, contradiction.

If $x_{1} \neq(1,0)$ then $y_{1}=(1,0)$ and $\gamma^{2}(1, x, y) \geqslant 1$ since $y$ is a best reply against $x$. Hence $\gamma^{1}(1, x, y) \leqslant-1<\nu_{1}^{1}$, contradiction.

b) Suppose that $(x, y)$ is a stationary limiting average equilibrium for initial state 2. If $y_{2}=(1,0)$ then $x_{2}=(1,0)$ and $\gamma^{2}(2, x, y)=1$ since $x$ is a best reply against $y$ for player 1 . But then $y$ is no best reply against $x$ for player 2 , contradiction.

If $y_{2} \neq(1,0)$ then player 1 can achieve a limiting average reward at least 0 by playing $(0,1)$ in state 2 , playing $(1,0)$ in state 3 and by playing in state 1 the action $(1,0)$ if $y_{1}(1) \geqslant / / 2$ or $(0,1)$ if $y_{1}(1)<1 / 2$. Hence $\gamma^{2}(2, x, y) \leqslant 0<v_{2}^{2}$, contradiction.

c) Suppose that $(x, y)$ is a stationary limiting average equilibrium for initial state 3. If $x_{3}=(1,0)$, then $y_{3}=(1,0)$ and $\gamma^{1}(3, x, y)=1$ since $y$ is a best reply against $x$ for player 2 . But then $x$ is no best reply for player 1 against $y$, for by playing $(0,1)$ against $y$ in starting state 3 player 1 could get limiting average reward 2 , contradiction.

If $x_{3} \neq(1,0)$ then player 2 can get limiting average reward at least 0 by playing $(1,0)$ in state $2,(0,1)$ in state 3 and $(1 / 2,1 / 2)$ in state 1 . Hence $\gamma^{1}(3, x, y) \leqslant 0<v_{3}^{1}$, contradiction.

However, although for none of the initial states there is a stationary limiting average equilibrium, an almost stationary limiting average $t$-equilibrium exists (for all initial states).

This follows from lemma 2.3.4, because it can be verified that for each $\beta \in[0,1)$ the pair $\left(x^{\beta}, y^{\beta}\right)$ defined below is a stationary $\beta$-discounted equilibrium and $\left(x^{1}, y^{\prime}\right)=\lim _{\beta \uparrow 1}\left(x^{\beta}, y^{\beta}\right)$ satisfies the condition of lemma 2.3.4.

For $\beta \in[0,1)$ define $\left(x^{\beta}, y^{\beta}\right)$ by:

$$
\begin{aligned}
& x_{1}^{\beta}=\left(\frac{3-\beta-\sqrt{9-8 \beta}}{\beta}, \frac{-3+2 \beta+\sqrt{9-8 \beta}}{\beta}\right), \\
& x_{2}^{\beta}=\left(\frac{3-\sqrt{9-8 \beta}}{2 \beta}, \frac{-3+2 \beta+\sqrt{9-8 \beta}}{2 \beta}\right), \\
& x_{3}^{\beta}=\left(\frac{3-\sqrt{9-8 \beta}}{2 \beta}, \frac{-3+2 \beta+\sqrt{9-8 \beta}}{2 \beta}\right), \\
& y_{1}^{\beta}=(1 / 2,1 / 2), \\
& y_{2}^{\beta}=\left(\frac{3-\sqrt{9-8 \beta}}{2 \beta}, \frac{-3+2 \beta+\sqrt{9-8 \beta}}{2 \beta}\right),
\end{aligned}
$$




$$
y_{3}^{\beta}=\left(\frac{3-\sqrt{9-8 \beta}}{2 \beta}, \frac{-3+2 \beta+\sqrt{9-8 \beta}}{2 \beta}\right) .
$$

It follows that $x^{1}=((1,0),(1,0),(1,0))$ and $y^{1}=((1 / 2,1 / 2),(1,0),(1,0))$. Furthermore we derive that:

$$
\begin{aligned}
& \gamma_{\beta}^{1}\left(x^{\beta}, y^{\beta}\right)=\left(0, \frac{3-4 \beta-\sqrt{9-8 \beta}}{2 \beta}, \frac{-3+4 \beta+\sqrt{9-8 \beta}}{2 \beta}\right), \\
& \gamma_{\beta}^{2}\left(x^{\beta}, y^{\beta}\right)=\left(0, \frac{-3+4 \beta+\sqrt{9-8 \beta}}{2 \beta}, \frac{3-4 \beta-\sqrt{9-8 \beta}}{2 \beta}\right), \\
& \gamma^{1}\left(x^{1}, y^{1}\right)=(0,-1,1)=\lim _{\beta \uparrow 1} \gamma_{\beta}^{1}\left(x^{\beta}, y^{\beta}\right)=y^{1}, \\
& \gamma^{2}\left(x^{1}, y^{1}\right)=(0,1,-1)=\lim _{\beta \uparrow 1} \gamma_{\beta}^{2}\left(x^{\beta}, y^{\beta}\right)=y^{2} .
\end{aligned}
$$

Since the states 1, 2 and 3 are each ergodic sets with respect to $\left(x^{1}, y^{1}\right)$, we can apply lemma 2.3 .4 for each state.

\section{$2.4(\epsilon-)$ EASY INITIAL STATES IN THE ZERO-SUM CASE}

In this section we show that for each player there are easy states in any zerosum stochastic game. For all states with minimal limiting average value, player 1 has a stationary limiting average c-optimal strategy. For the states with maximal limiting average value we give a sufficient condition for player 1 to have a stationary limiting average $\epsilon$-optimal strategy. Similar results hold for player 2.

\subsubsection{DEFINITION}

A state $s$ is called an (e-)easy initial state for player $k$ if player $k$ has a stationary limiting average ( $\epsilon$-)optimal strategy for the game starting in $s$.

It is clear that the set of e-easy initial states for player $k$ contains the set of easy initial states for this player. However, there need not be states which are easy for both players, whereas all states may be $\epsilon$-easy for both players. Hence the set of $\epsilon$-easy states for a player is generally larger than the set of easy states for this same player. The following theorem is due to Tijs \& Vrieze [1986].

\subsubsection{THEOREM}

For any zero-sum stochastic game each player has a non-empty set of easy initial states.

The proof presented by Tijs \& Vrieze [1986] for this theorem is based on the result of Bewley \& Kohlberg [1976] who showed that there are solutions for the $\beta$-discounted zero-sum case which can be written as power series in fractional powers of $(1-\beta)$ (cf. theorem 1.7.5). We give a new proof for theorem 2.4 .2 based on the results derived in section 2.2 . 


\subsubsection{REMARK}

In this section $\left\{x^{\beta}: \beta \in[0,1)\right\}$ is a sequence of stationary $\beta$-discounted optimal strategies for player 1 , which converges to $x^{1}$. Likewise we have $\left\{y^{\beta}: \beta \in[0,1)\right\}$ for player 2 converging to $y^{\mathbb{1}}$.

\section{Proof of THEOREM 2.4.2:}

We prove the existence of easy initial states for player 1 . Let $y^{*}$ be a stationary limiting average best reply against $x^{1}$ (cf. remark 2.4 .3 and lemma 1.6.2). Let $Z^{\beta}, V^{k}, S^{1}, \ldots, S^{H}$ etc. be as in definition 2.2 .1 for the sequence $\left\{\left(x^{\beta}, y^{*}\right): \beta \in[0,1)\right\}$.

By corollary 2.2 .7 there is $h^{1} \in\{1,2, \ldots, H\}$ with $\gamma^{1 h^{1}}\left(x^{3}, y^{*}\right) \geqslant \max _{s \in S} V_{s}^{1}$. For all $s \in S$ we also have, using theorem 1.7.7, that:

$$
V_{s}^{1}=\lim _{\beta \uparrow 1} \gamma_{\beta}^{1}\left(s, x^{\beta}, y^{*}\right) \geqslant \lim _{\beta \uparrow 1} v_{\beta}^{1}(s)=v_{s}^{1} .
$$

Hence for all $s \in S^{h^{\prime}}$ and all $\sigma \in \Sigma$ we have:

$$
\gamma^{\natural}\left(s, x^{\prime}, \sigma\right) \geqslant \gamma^{\natural}\left(s, x^{1}, y^{*}\right) \geqslant \max _{t \in S} V_{t}^{\sharp} \geqslant \max _{t \in S} v_{t}^{1} .
$$

We conclude that $x^{1}$ is limiting average optimal for all $s \in S^{h^{2}}$.

Observe that by the above proof it follows that any limit of stationary $\beta$ discounted strategies ( $\beta$ tending to 1$)$ is limiting average optimal for some starting states. Moreover, for player 1 we found that among those easy initial states there are states for which the limiting average value is maximal. Similarly we conclude that for player 2 the strategy $y^{1}$ is limiting average optimal for some initial states for which the limiting average value is minimal.

The converse is not true: if for a state the limiting average value is maximal (or minimal), then this does not imply that player 1 (2) has a stationary limiting average optimal strategy. This is demonstrated by the next example.

\subsubsection{EXAMPLE}

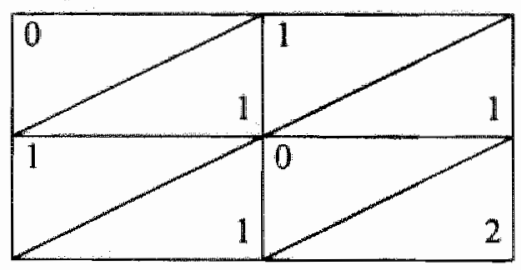

State 1

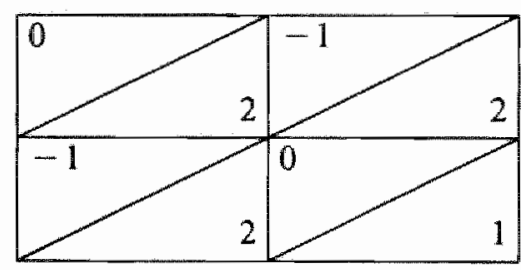

State 2

Payoffs are again those to player 1 to be paid by player 2 . It is easy to verify that for this stochastic game the limiting average value $v^{\mathbb{1}}=(0,0)$. For player 1 (2) a stationary limiting average $\epsilon$-optimal strategy is $x^{\epsilon}=((1,0),(1-\epsilon, \epsilon))$ (resp. $\left.y^{\epsilon}=((1-\epsilon, \epsilon),(1,0))\right)$.

It is clear that for initial state 2 (1) player 1 (resp. 2) has no stationary limiting 
average optimal strategy. So, although the value is maximal as well as minimal for both starting states, each player has a stationary limiting average optimal strategy for just one of them.

As we have just remarked, there are easy states for player 1 among the states with maximal limiting average vallue. The next example illustrates that there need not be easy initial states for player 1 among the states with minimal limiting average value.

\subsubsection{EXAMPLE}

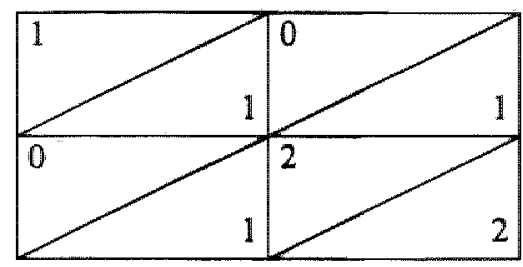

State 1

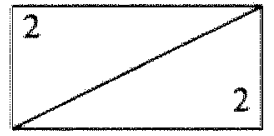

State 2

Payoffs are again those to player 1 to be paid by player 2 .

For this stochastic game the limiting average value $v^{1}$ equals $(1,2)$. For player 1 a stationary limiting average $\epsilon$-optimal strategy is given by $x^{\star}:=(1-\epsilon, \epsilon)$, the mixed action to be used in state 1 . For player 2 a stationary limiting average optimal strategy is $y^{*}:=(1,0)$.

It is easy to see that for state 1 , the state with minimal limiting average value, player 1 has no stationary limiting average optimal strategy.

Nevertheless, states for which the limiting average value is maximal or minimal are special, as is illustrated in the following two theorems.

\subsubsection{THEOREM}

Let $v^{\min }:=\min _{s \in S} v_{s}^{1}$ and let $S^{\min }:=\left\{s \in S: v_{s}^{\mathbb{1}}=v^{\min }\right\}$.

All states in $S^{\min }$ are c-easy for player 1 .

\section{Proof:}

Let $y \in Y$ be arbitrary.

Using that $\gamma_{\beta}^{\prime}\left(x^{\beta}, y\right) \geqslant v_{\beta}^{1}$ (cf. remark 2.4.3) and using theorem 1.7.3 we have:

$$
(1-\beta) r^{1}\left(x^{\beta}, y\right)+\beta P\left(x^{\beta}, y\right) v_{\beta}^{1} \geqslant v_{\beta}^{1} \text { for all } \beta \in[0,1) \text {. }
$$

Multiplying this inequality with $Q\left(x^{\beta}, y\right)$ gives that:

$$
Q\left(x^{\beta}, y\right) r^{1}\left(x^{\beta}, y\right)>Q\left(x^{\beta}, y\right) v_{\beta}^{1} \text { for all } \beta \in[0,1) \text {. }
$$

Hence for $\beta$ such that $\left\|v_{\beta}-v^{1}\right\|<\epsilon$ (cf. theorem 1.7.7) we have, by lemma 1.5.5:

$$
\begin{aligned}
\gamma^{1}\left(x^{\beta}, y\right) & =Q\left(x^{\beta}, y\right) r^{1}\left(x^{\beta}, y\right) \geqslant Q\left(x^{\beta}, y\right) v_{\beta}^{1} \\
& \geqslant Q\left(x^{\beta}, y\right) v^{1}-\epsilon 1_{z} \geqslant v^{\min }-\epsilon 1_{z} .
\end{aligned}
$$


Hence $x^{\beta}$, with $\beta$ such that $\left\|v_{\beta}^{1}-v^{1}\right\|<\epsilon$, is a stationary limiting average $\boldsymbol{c}$ optimal strategy for player 1 for all initial states in $S^{\min }$.

Recall that by theorem 1.7.5 (Bewley \& Kohlberg [1976]) we may assume that there are $N \in \mathbb{N}, x_{0} \underset{s=1}{\underset{x}{x}} \Delta^{m_{s}}, x_{\|}, x_{2}, \ldots, \in \underset{s=1}{\underset{z}{x}} \mathbb{R}^{m_{s}}$, such that:

$$
x^{\beta}=\sum_{n=0}^{\infty} x_{n}(1-\beta)^{n / N} \text { for all } \beta \text { close to } 1 .
$$

Since $x^{\beta} \in X$, it holds that: $x^{1}=x_{0} ; \sum_{i=1}^{m_{4}} x_{n s}(i)=0$ for all $n \geqslant 1$ and for all $s \in S$; if $x_{0 . s}(i)=x_{1 s}(i)=\ldots=x_{n-15}(i)=0$, then $x_{n s}(i) \geqslant 0$, for each $s \in S$ and each $n \geq 1 ; \sum_{n=0} x_{n}(1-\beta)^{n / N} \in X$ for each $l \in \mathbb{N}$. We use these facts to examine limiting average $\epsilon^{-}$-optimality for player 1 in states with maximal limiting average value.

\subsubsection{DEFINITION}

Let $v^{\max }:=\max _{s \in S_{s}} v_{s}^{1}$ and let $S^{\max }:=\left\{s \in S: v_{s}^{1}=v^{\max }\right\}$.

Let $S^{*}:=\left\{s \in S^{\max }: x^{1}\right.$ is limiting average optimal for intial state $\left.s\right\}$.

Define $\bar{x}^{\beta} \in X$ by $\bar{x}_{s}^{\beta}:=x_{s}^{1}$ for $s \in S^{*}$ and $\bar{x}_{s}^{\beta}:=\sum_{n=0}^{N-\mathbb{1}} x_{n}(1-\beta)^{n / N}$ for $s \in S \backslash S^{*}$.

Let $\bar{y} \in Y$ be a stationary limiting average best reply against $\bar{x}^{\beta}$, for all $\beta$ close to $\ell$.

Define $S^{* *}:=S^{*} \cup\left\{E \subset S^{\max } \backslash S^{*}:\right.$ E ergodic with respect to $\left.\left(x^{\beta}, y\right)\right\}$.

Define $A:=S^{\max } \backslash S^{* * *}$.

\subsubsection{THEOREM}

a) $S_{\beta}^{*} \neq \varnothing$.

b) $\bar{x}^{\beta}$ is limiting average c-optimal for initial states in $S^{* *}$ for $\beta$ close to $I$.

c) If $\lim _{\beta \uparrow !}(1-\beta)\left(I^{A}-\beta P(\bar{x}, \bar{y})^{A}\right)^{-1}=0$, then $\bar{x}^{\beta}$ is limiting average coptimal for all initial states in $S^{\mathrm{mux}}$ for $\beta$ close to 1 . (Here the superscript $A$ denotes the restriction to rows and columns corresponding with states in $A$ )

\section{Proof:}

a) In the above we noticed that, by the proof of theorem 2.4 .2 , there are states in $S^{\max }$ which are easy for player 1, i.e. for which $x^{1}$ is limiting average optimal. Hence $S^{*} \neq \varnothing$.

b) We already noticed that:

$$
(1-\beta) r^{1}\left(x^{\beta}, y\right)+\beta P\left(x^{\beta}, y\right) v_{\beta}^{1} \geqslant v_{\beta}^{1} \text { for all } y \in Y \text {. }
$$

Letting $\beta$ tend to 1 , this gives us that $P\left(x^{1}, y\right) v^{1} \geqslant v^{1}$ for all $y \in Y$. Hence, if player 1 uses $x^{1}$ in $S^{\max }$, then play will remain in $S^{\max }$ with probability 1 , no matter what strategy is used by player 2 . 
From the fact that for initial states in $S^{*}$ the strategy $x^{1}$ is limiting average optimal, one can conclude that, if player 1 uses $x^{1}$ for initial states in $S^{*}$, then play will remain within $S^{*}$ with probability 1 . Again this does not depend on the strategy used by player 2 .

We define (cf. theorem 1.7.5):

$$
x^{\beta}(N):=\sum_{n=0}^{N-1} x_{n}(1-\beta)^{n / N} \text { and } \underline{x}^{\beta}(N):=\sum_{n=N+1}^{\infty} x_{n}(1-\beta)^{n / N} .
$$

Notice that $\lim _{\beta \uparrow 1}(1-\beta)^{-1} \underline{x}^{\beta}(N)=0$ and that $x_{s}^{\beta}(N)=\bar{x}_{s}^{\beta}$ for $s \in S \backslash S^{*}$.

We show:

For any set $E \subset S^{\max } \backslash S^{*}$, such that $E$ is ergadic with respect to $\left(\bar{x}^{\beta}, \bar{y}\right)$, we have that $\gamma^{1}\left(s, \bar{x}^{\beta}, \bar{y}\right)>v^{\max }-\epsilon$ for any initial state $s \in E$ and $\beta$ clase to 1 . For $\beta$ close to 1 we have $y_{\beta}^{1} \leqslant(1-\beta) r^{1}\left(x^{\beta}, y\right)+\beta P\left(x^{\beta}, y\right)$ and hence:

$$
\begin{aligned}
v_{\beta}^{1} \leqslant & (1-\beta) r^{1}\left(x^{\beta}(N), \bar{y}\right)+\beta P\left(x^{\beta}(N), \bar{y}\right) v_{\beta}^{1} \\
& +(1-\beta)^{2} r^{1}\left(x_{N}, \bar{y}\right)+\beta(1-\beta) P\left(x_{N}, \bar{y}\right) v_{\beta}^{1} \\
& +(1-\beta) r^{1}\left(x^{\beta}(N), \bar{y}\right)+\beta P\left(x^{\beta}(N), \bar{y}\right) v_{\beta}^{1} .
\end{aligned}
$$

Let $Q_{E}^{\beta}$ denote the restriction of $Q\left(x^{\beta}(N), y\right)$ to rows corresponding with states in $E$. Hence $Q_{E}^{\beta}$ has size $|E| \times z$. Multiplying the above inequality by $Q_{E}^{\beta}$ yields (cf. lemma 1.5.2 (e)):

$$
\begin{aligned}
Q_{E}^{\beta} v_{\beta}^{1} \leqslant & (1-\beta) Q_{E}^{\beta} r^{1}\left(x^{\beta}(N), \bar{y}\right)+\beta Q_{E}^{\beta} v_{\beta}^{1} \\
& +(1-\beta)^{2} Q_{E}^{\beta} r^{1}\left(x_{N}, \bar{y}\right)+\beta(1-\beta) Q_{E}^{\beta} P\left(x_{N}, \bar{y}\right) v_{\beta}^{1} \\
& +(1-\beta) Q_{E}^{\beta} r^{1}\left(x^{\beta}(N), \bar{y}\right)+\beta Q_{E}^{\beta} P\left(x^{\beta}(N), \bar{y}\right) v_{\beta}^{1} .
\end{aligned}
$$

Hence:

$$
\begin{aligned}
Q_{E}^{\beta} v_{\beta}^{1} \leqslant & Q_{E}^{\beta} r^{1}\left(x^{\beta}(N), \bar{y}\right) \\
& +(1-\beta) Q_{E}^{\beta} r^{1}\left(x_{N}, \bar{y}\right)+\beta Q_{E}^{\beta} P\left(x_{N}, \bar{y}\right) v_{\beta}^{\beta} \\
& +Q_{E}^{\beta} r^{1}\left(x^{\beta}(N), \bar{y}\right)+\beta(1-\beta)^{-1} Q_{E}^{\beta} P\left(x^{\beta}(N), \bar{y}\right) v_{\beta}^{1}
\end{aligned}
$$

It can be verified that:

$$
\begin{aligned}
& \lim _{\beta \uparrow 1}(1-\beta) Q_{E}^{\beta} r^{1}\left(x_{N}, \bar{y}\right)=0, \lim _{\beta \uparrow 1} \beta Q_{E}^{\beta} P\left(x_{N}, \bar{y}\right) v_{\beta}^{1} \leqslant 0 \\
& \lim _{\beta \uparrow 1} Q_{E}^{\beta} r^{\prime}\left(x^{\beta}(N), \bar{y}\right)=0, \lim _{\beta \uparrow 1} \beta(1-\beta)^{-1} Q_{E}^{\beta} P\left(x^{\beta}(N), \bar{y}\right) v_{\beta}^{1}=0 .
\end{aligned}
$$

To show that $\lim _{\beta \uparrow 1} \beta Q Q_{E}^{\beta} P\left(x_{N}, \bar{y}\right) v_{\beta} \leqslant 0$, take $\delta>0$. For $s \in E$ we have:

$$
\sum_{i=1}^{z} p\left(t \mid s, x_{N_{s}}, \bar{y}_{s}\right) v_{\beta}^{l}(t)=\sum_{i=1}^{m_{s}} \sum_{t=1}^{z} x_{N_{s}}(i) p\left(t \mid s, i, \bar{y}_{s}\right) v_{\beta}^{1}(t)
$$

For $\beta$ close to 1 we have: 


$$
\sum_{i=1}^{z} p\left(t \mid s_{s} i_{y} \bar{y}_{s}\right) v \beta(t)<v^{\max }+\delta \text { for all } i \in\left\{1,2, \ldots, m_{s}\right\}
$$

For it with $x_{N_{s}}(i)<0$, we have $i \in \operatorname{Car}\left(x_{s}^{\beta}(N)\right)$, as remarked above, and hence for those $i$ and $\beta$ close to 1 :

$$
\sum_{t=1}^{x} p\left(t \mid s, i_{s} \bar{y}_{s}\right) v_{\beta}(t)=\sum_{t \in E} p\left(t \mid s, i_{,} \bar{y}_{s}\right) \nu_{\beta}(t) \geqslant v^{\max }-\delta .
$$

Combining these inequalities yields:

$$
\begin{aligned}
& \sum_{i=1}^{m_{s}} \sum_{i=1}^{z} x_{N s}(i) p\left(t \mid s, i, \bar{y}_{s}\right) v_{\beta}^{\mathbb{N}}(t)<\sum_{i, x_{s s}(i)<0} x_{N_{s}}(i)\left(v^{\max }-\delta\right)+\sum_{i, x_{w s}(i) \geqslant 0} x_{N_{s}}(i)\left(v^{\max }+\delta\right) \\
& =28 \sum_{i, x_{N}(i) \geqslant 0} x_{M S}(i) \text {. }
\end{aligned}
$$

Since $\delta>0$ was arbitrary, we conclude that $\lim _{\beta \uparrow 1} \beta P\left(x_{N}, \bar{y}\right) v_{\beta}^{1} \leqslant 0$, and hence that $\lim _{\beta \uparrow 1} \beta Q_{E}^{\beta} P\left(x_{N}, \bar{y}\right) v_{\beta}^{1} \leqslant 0$.

Altogether we have:

$$
\begin{aligned}
v^{\max } 1^{E}=\lim _{\beta \uparrow 1} Q_{E}^{\beta} v_{\beta}^{1} \leqslant & \lim _{\beta \uparrow \mathbb{1}} Q_{E}^{\beta} r^{1}\left(x^{\beta}(N), \bar{y}\right) \\
& +\lim _{\beta \uparrow 1}(1-\beta) Q_{E}^{\beta} r^{1}\left(x_{N}, \bar{y}\right)+\lim _{\beta \uparrow 1} \beta Q_{E}^{\beta} P\left(x_{N}, \bar{y}\right) v_{\beta}^{1} \\
& +\lim _{\beta \uparrow 1} Q_{E}^{\beta} r^{1}\left(x^{\beta}(N), \bar{y}\right)+\lim _{\beta \uparrow 1} \beta(1-\beta)^{-1} Q_{E}^{\beta} P\left(x^{\beta^{\prime}}(N), \bar{y}\right) v_{\beta}^{\mathbb{B}} \\
& =\lim _{\beta \uparrow 1} Q_{E}^{\beta} r^{1}\left(x^{\beta}(N), \bar{y}\right)=\lim _{\beta \uparrow 1} \gamma^{1}\left(\bar{x}^{\beta}, \bar{y}\right) .
\end{aligned}
$$

Because $\bar{y}$ is a limiting average best reply against $\bar{x}^{\beta}$, for all $\beta$ close to 1 , this implies that $\bar{x}^{\beta}$ is a limiting average $\epsilon$-optimal strategy for all initial states in $E$, for $\beta$ close to 1 . This result together with (a) shows that $\bar{x}^{\beta}$ is limiting average $\epsilon$-optimal for all initial states in $S^{* *}$, for $\beta$ close to 1 .

c) We show:

If $\lim _{\beta \uparrow \|}(1-\beta)\left(I^{A}-\beta P\left(x^{\beta}, \bar{y}\right)^{A}\right)^{-1}=0$, then $\bar{x}^{\beta}$ is limiting average e-optimal for all initial states in $A$, for $\beta$ close to 1 .

Let $v_{\beta}^{A}, r^{1}\left(x^{\beta}, \bar{y}\right)^{A}, 1^{A}$ (etc.) denote the restriction of $v_{\beta}^{\mathbb{B}}, r^{1}\left(x^{\beta}, \bar{y}\right)$ and $1^{A}$ to coordinates in $A$. Let $v_{\beta}^{A C}$ (etc.) denote the restriction to coordinates in $A^{c}:=S \backslash A$. Also let $P\left(x^{\beta}, \bar{y}\right)^{A}$ (etc.) denote the restriction of $P\left(x^{\beta}, \bar{y}\right)$ to rows and columns corresponding with states in $A$; let $P\left(x^{\beta}, \bar{y}\right)_{A}$ (etc) denote restriction of $P\left(x^{\beta}, \bar{y}\right)$ only to rows corresponding with states in $A$.

As above we start off with: $v_{\beta}^{1} \leqslant(1-\beta) r^{1}\left(x^{\beta}, \bar{y}\right)+\beta P\left(x^{\beta}, \bar{y}\right) v_{\beta}^{1}$.

This time we derive: 


$$
\begin{aligned}
v_{\beta}^{L A} \leqslant & (1-\beta) r^{1}\left(x^{\beta}, \bar{y}\right)^{A}+\beta P\left(x^{\beta}(N), \bar{y}\right)^{A} v_{\beta}^{1 A}+\beta P\left(x^{\beta}(N), \bar{y}\right)^{A C_{1}} v_{\beta}^{L C} \\
& +\beta(1-\beta) P\left(x_{N}, \bar{y}\right)_{A} v_{\beta}+\beta P\left(x^{\beta}(N), \bar{y}\right)_{A} v_{\beta}^{1} .
\end{aligned}
$$

Substracting $\beta P\left(x^{\beta}(N), \bar{y}\right)^{A} \nu_{\beta}^{A A}$ from both sides, multiplying both sides with $\left(I^{A}-\beta P\left(x^{\beta}(N), \bar{y}\right)^{A}\right)^{-1}$, which exists because $x^{\beta}(N) \in X$, and by taking limits we obtain:

$$
\begin{aligned}
v^{\max } 1^{A}=\lim _{\beta \uparrow 1} v_{\beta}^{L A} \leqslant & \lim _{\beta \uparrow 1} \beta\left(I^{A}-\beta P\left(x^{\beta}(N), \bar{y}\right)^{A}\right)^{-1} P\left(x^{\beta}(N), \bar{y}\right)^{A C_{v_{\beta}}{ }^{A C}} \\
& +\lim _{\beta \uparrow 1}(1-\beta)\left(I^{A}-\beta P\left(x^{\beta}(N), \bar{y}\right)^{A}\right)^{-1}\left[r^{1}\left(x^{\beta}, \bar{y}\right)^{A}+\beta P\left(x_{N}, \bar{y}\right)_{A} v_{\beta}^{1}\right. \\
& \left.+\beta(1-\beta)^{-1} P\left(x^{\beta}(N), \bar{y}\right)_{A} v_{\beta}^{1}\right] .
\end{aligned}
$$

Observe that each term within the square brackets is bounded uniformly in $\beta$. Hence the condition in (c) gives:

$$
\begin{aligned}
v^{\max } 1^{A} & \leqslant \lim _{\beta \uparrow 1} \beta\left(I^{A}-\beta P\left(x^{\beta}(N), \bar{y}\right)^{A}\right)^{-1} P\left(x^{\beta}(N), \bar{y}\right)^{A C_{v} v_{\beta}^{A C}} \\
& =\lim _{\beta \uparrow 1}\left(I^{A}-\beta P\left(x^{\beta}(N), \bar{y}\right)^{A}\right)^{-1} P\left(x^{\beta}(N), \bar{y}\right)^{A C} v^{1 A C}
\end{aligned}
$$

Now we will use the following relation, which holds for any square matrix $P$ such that $(I-P)^{-1}$ exists:

$$
(I-\beta P)^{-1}=(I-P)^{-1}-(1-\beta)(I-\beta P)^{-1} P(I-P)^{-1},
$$

This can easily be verified by multiplying both sides with $(I-P)$.

Applying this for $P=P\left(x^{\beta}(N), \bar{y}\right)^{A}$, using that $P\left(x^{\beta}(N), \bar{y}\right)^{A}\left(I^{A}-P\left(x^{\beta}(N), \bar{y}\right)^{A}\right)^{-1} P\left(x^{\beta}(N), \bar{y}\right)^{A C}$ is bounded and that $\lim _{\beta \uparrow 1}(\mathbb{1}-\beta)\left(I^{A}-\beta P\left(x^{\beta}(N), \bar{y}\right)^{A}\right)^{-1}=0$, yields:

$$
v^{\max } 1^{A} \leqslant \lim _{\beta \uparrow 1}\left(I^{A}-P\left(x^{\beta}(N), \bar{y}\right)^{A}\right)^{-1} P\left(x^{\beta}(N), \bar{y}\right)^{A C} v^{L A C} .
$$

Since $v^{1 A C} \leqslant v^{\max } 1^{A C}$, the inequality sign in the above inequality can be replaced by an equality sign. Next observe that entry $(s, t)$ of matrix $\left(I^{A}-P\left(x^{\beta}(N), \bar{y}\right)^{A}\right)^{-1} P\left(x^{\beta}(N), \bar{y}\right)^{A C}$ denotes the total probability of ever entering $A^{c}$ through state $t$ when starting in $s$. Hence the probability of entering $S$ when starting in $A$ is close to 1 for $\beta$ sufficiently near 1 .

Thus we have that $\gamma^{1}\left(s, \bar{x}^{\beta}, \bar{p}\right)>v^{\max }-\epsilon$ for each $s \in A$, for $\beta$ close to 1 .

\subsubsection{COROLLARY}

If for a zero-sum stochastic game we have that $S=S^{\min }=S^{\max }$, then both players have stationary limiting average e-optimal strategies.

This result can also be found in Bewley \& Kohlberg [1978] or Vrieze [1987-a].

\subsubsection{REMARK}

Observe that each entry of $\left(I^{A}-\beta P\left(x^{\beta}, \mathcal{y}\right)^{A}\right)^{-1}$ can be written as $\sum_{n=1}^{\infty} c_{n}(1-\beta)^{n / N}$ 
with $l \in \mathbb{Z}$ and $c_{n} \in \mathbb{R}$. Then the condition in theorem 2.4 .8 (c) is fulfilled if and only if $I>-N$ for each entry. This holds for instance if all states $A$ are transient with respect to $\left(x^{1}, y\right)$, since in this case $\lim _{\beta \uparrow 1}\left(I^{A}-\beta P(x, y)^{A}\right)^{-1}$ exists.

If $P\left(x^{\beta}, \bar{y}\right)_{s}^{A C} \neq 0$ for each $s \in A$, then the condition of theorem 2.4 .8 (c) automatically holds because $\left\|P^{m}\left(x^{\beta}, y\right)^{A}\right\| \leqslant c\left(1-(1-\beta)^{1 / N}\right)^{n}$ for some constant $c \in \mathbb{R}$.

The next example shows that there may be states which are neither 6 -easy for player 1 nor for player 2 and that for each player $S^{\min } \cup S^{\max }$ may be the set of all his e-easy initial states.

\subsubsection{EXAMPLE}

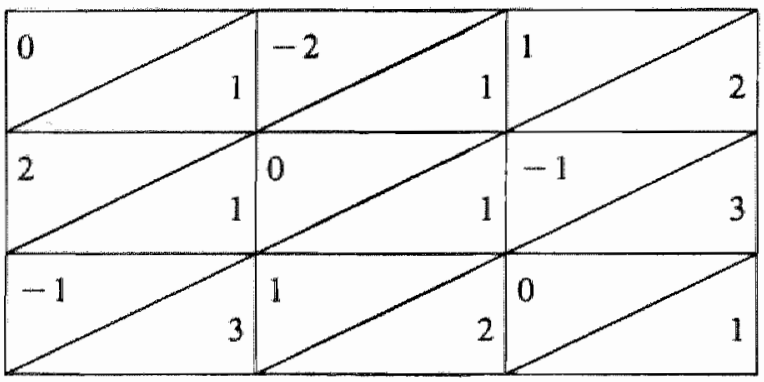

State 1

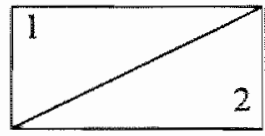

State 2

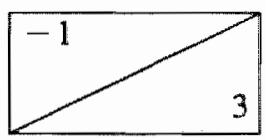

State 3

Payoffs are again those to player I to be paid by player 2 .

The unique stationary $\beta$-discounted optimal strategies for this stochastic game are (for starting state 1) given by:

$$
x^{\beta}=y^{\beta}=(1 /(4-2 \beta), 1 /(4-2 \beta),(1-\beta) /(2-\beta)) .
$$

For all $\beta \in[0,1)$ we have $y^{\beta}=(0,1,-1)$, hence also $y^{1}=(0,1,-1)$.

So $S^{\max }=\{2\}$ and $S^{\min }=\{3\}$.

State $\mathbb{1}$ is neither -easy for player 1 nor for player 2 , since each of them faces a 'kind of big match' for starting state 1 (cf. example 1.7.4).

\subsubsection{REMARK}

For a zero-sum stochastic game a strong initial state is not-necessarily an e-easy initial state.

This is illustrated by the examples 1.7 .4 and 2.3.8. In these examples state $\mathbb{1}$ is a strong initial state but state 1 is not e-easy for player 1 . 


\section{Chapter 3}

\section{Existence of limiting average $\boldsymbol{\epsilon}$-equillibria}

\subsection{INTRODUCTION}

Since Mertens \& Neyman [1981] showed that the limiting average value exists for any zero-sum stochastic game, the major remaining problem in stochastic game theory is that of existence of limiting average c-equilibria for the general-sum case.

By putting extra conditions on the payoffs and/or transition structure of the stochastic game, several authors have shown that limiting average ( $\epsilon$-)equilibria exist for subclasses of stochastic games (cf. chapter 4). In this chapter we present sufficient conditions for the existence of (almost stationary) limiting average $\epsilon$-equilibria. However, our conditions are of a more general nature. We do not put conditions on the payoff/transition structure from the start, but our conditions are formulated in terms of asymptotic properties of sequences of stationary $\beta$-discounted equilibria. Remember that stationary $\beta$-discounted equilibria exist for any general-sum stochastic game. In chapter 4 we show that our conditions are automatically fulfilled for several of the subclasses that have been examined in literature. It is not clear whether our conditions hold for any general-sum stochastic game. Nevertheless our approach shows that in general the set of strong initial states is larger than the union of ergodic sets for which ' $\gamma^{k h}\left(x^{1}, y^{1}\right) \geqslant V^{k h}$ for $k=1,2$ ' (cf. lemma 2.3.4).

The results of this chapter have been derived from Thuijsman \& Vrieze [1989-c].

\subsection{FINDING MORE STRONG INITIAL STATES}

\subsubsection{REMARK}

In this section let $\left\{\left(x^{\beta}, y^{\beta}\right) ; \beta \in[0,1)\right\}$ be a sequence of stationary $\beta$-discounted equilibria with $\lim _{\beta \uparrow 1}\left(x^{\beta}, y^{\beta}\right)=\left(x^{1}, y^{1}\right)$ and which furthermore suits definition 2.2.I.

Observe that all results in section 2.3 were derived for such a sequence, so we can use those results here. We introduce some more notation.

\subsubsection{DEFINITION}

For our sequence $\left\{\left(x^{\beta}, y^{\beta}\right): \beta \in[0,1)\right\}$ we define:

$I:=\left\{h \in\{1,2, \ldots, H\}: \gamma^{1 / h}\left(x^{1}, y^{1}\right) \geqslant V^{1 / h}\right.$ and $\left.\gamma^{2 h}\left(x^{1}, y^{1}\right) \geqslant V^{2 h}\right\}$, 
$I_{0}:=\left\{h \in\{1,2, \ldots, H\}: \gamma^{1 / h}\left(x^{1}, y^{1}\right)=V^{1 h}\right.$ and $\left.\gamma^{2 h}\left(x^{1}, y^{1}\right)=V^{2 h}\right\}$,

$I_{1}:=\left(h \in\{1,2, \ldots, H\}: \gamma^{1 / h}\left(x^{1}, y^{11}\right)<V^{1 / \hbar}\right\}$,

$I_{2}:=\left\{h \in\{1,2, \ldots, H\}: \gamma^{1 h}\left(x^{1}, y^{1}\right) \geq V^{1 / h}\right.$ and $\left.\gamma^{2 h}\left(x^{1}, y^{1}\right)<V^{2 h}\right\}$,

$E:=\{s \in S:$ there is a limiting average e-equilibrium for initial state $s$, for all $\epsilon>0\}$.

Observe that $I \neq \varnothing$ by lemma 2.3 .6 and $I_{0} \subset I \subset E$ by lemma 2.3 .4 , hence $E \neq \varnothing$ (cf. theorem 2.3.5). Furthermore notice that $1, I_{1}$ and $I_{2}$ have empty intersections and $S=T \cup S^{I} \cup S^{\ell_{1}} \cup S^{I_{2}}$. Recall that $T$ is the set of states that are transient with respect to $\left(x^{1}, y^{1}\right)$ and that $S^{A}=\cup_{h \in A} S^{h}$ for any $A \subset\{1,2, \ldots, H\}$. The following example shows that $T, I_{1}$ and $I_{2}$ may all be empty, whereas $I_{0}$ does not need to be equal to $I$.

\subsubsection{EXAMPLE}

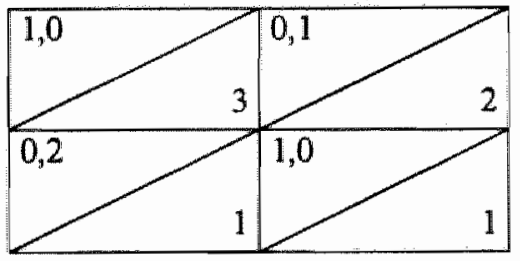

State 1

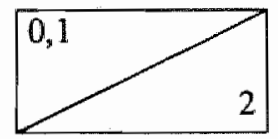

State 2

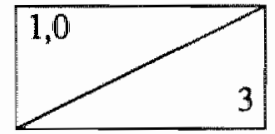

State 3

For this example the unique stationary $\beta$-discounted equilibria are given by (the mixed actions in state 1$):\left(x^{\beta}, y^{\beta}\right)=(((2-2 \beta) /(3-2 \beta), 1 /(3-2 \beta)),(1 / 2,1 / 2))$ and the corresponding $\beta$-discounted rewards are given by:

$\gamma_{\beta}^{\prime}\left(1, x^{\beta}, y^{\beta}\right)=1 / 2=v_{\beta}^{1}(1), \gamma_{\beta}^{2}\left(1, x^{\beta}, y^{\beta}\right)=z_{3}=v_{\beta}^{2}(1)$ for all $\beta \in[0,1)$.

Hence $\left(x^{1}, y^{1}\right)=((0,1),(1 / 2,1 / 2))$,

$\gamma^{1}\left(1, x^{1}, y^{1}\right)=1 / 2=V_{1}^{1}=v_{1}^{1}$ and $\gamma^{2}\left(1, x^{1}, y^{1}\right)=1>3 / 3=V_{1}^{2}=v_{1}^{2}$.

For $\left(x^{1}, y^{1}\right)$ there are three ergodic classes: $S^{1}=\{1\}, S^{2}=\{2\}, S^{3}=\{3\}$. It is easy to see that $T=\varnothing, I=\{1,2,3\}, I_{0}=\{2,3\}, I_{1}=I_{2}=\varnothing$.

Since for all ergodic sets this example suits the condition in lemma 2.3.4, the proof of that lemma supplies a limiting average c-equilibrium for this stochastic game.

It is clear that for any stochastic game for which $T \cup S^{I_{1}} \cup S^{I_{2}}=\varnothing$ for some sequence of stationary $\beta$-discounted equilibria $\left(x^{\beta} y^{\beta}\right)$, we can apply lemma 2.3 .4 to conclude the existence of a limiting average $\epsilon$-equilibrium (for each starting state). But how to proceed our search for a limiting average $\epsilon$ equilibrium if $T \cup S^{l_{1}} \cup S^{l_{2}} \neq \varnothing$ ? We deal with these matters in this section. 
We start by examining one more example and then we return to the general problem of existence. The objective of the discussion on next example is to create intuitive understanding for the lemmas that follow.

\subsubsection{EXAMPLE}

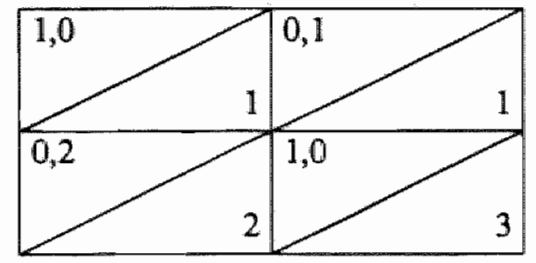

State 1

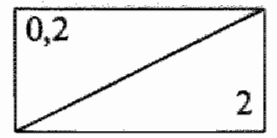

State 2

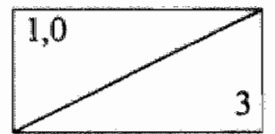

State 3

We already gave a brief discussion of this stochastic game (due to Sorin [1986]) in example 1.8.6. There however, we did not actually give a limiting average 6 equilibrium for this stochastic game. Recapitulate that the unique stationary $\beta$-discounted equilibria were given by:

$\left(x^{\beta}, y^{\beta}\right)=((2 /(3-\beta),(1-\beta) /(3-\beta)),(1 / 2,1 / 2))$.

The corresponding $\beta$-discounted rewards for starting state $\mathbb{1}$ were given by: $\gamma_{\beta}^{1}\left(1, x^{\beta}, y^{\beta}\right)=1 / 2=v_{\beta}^{1}(1)$ and $\gamma_{\beta}^{2}\left(1, x^{\beta}, y^{\beta}\right)=1 / 3=v_{\beta}^{2}(1)$ for all $\beta \in[0,1)$.

Hence $\left(x^{1}, y^{1}\right)=((1,0),(1 / 2,1 / 2)), V^{1}=v^{1}=(1 / 2,0,1)$ and $V^{2}=v^{2}=(2 / 3,2,0)$. So there are three ergodic sets with respect to $\left(x^{1}, y^{1}\right): S^{1}=\{1\}, S^{2}=\{2\}$ and $S^{3}=\{3\}$. It is clear that $I_{0}=\{2,3\}, T=\varnothing, I_{1}=\varnothing$ and $I_{2}=\{1\}$, because $\gamma^{11}\left(1, x^{1}, y^{1}\right)=1 / 2 \geqslant V_{1}^{1}$ and $\gamma^{2}\left(1, x^{1}, y^{1}\right)=1 / 2<V_{1}^{2}$.

Now observe that state 1 is transient with respect to $\left(x^{\beta}, y^{\beta}\right)$ for all $\beta \in[0,1)$, whereas state 1 is recurrent with respect to $\left(x^{1}, y^{1}\right)$. This means that for all $\beta \in[0,1)$ the payoffs in states 2 and 3 may partly determine the $\beta$-discounted rewards for state $]$ and hence the payoffs in states 2 and 3 may have their impact on $\lim _{\beta \uparrow \mid} \gamma\left(1, x^{\beta}, y^{\beta}\right)=V_{1}^{k}$ for $k=1$ and 2. Next observe that with respect to $\left(x^{1}, y^{1}\right)$ any play will remain in state 1 and hence $\gamma^{k}\left(1, x^{1}, y^{1}\right)$ is determined only by the payoffs in state 1 ; moreover $\gamma^{k}\left(1, x^{1}, y^{1}\right)=r^{k}\left(1, x^{1}, y^{1}\right)$. Let us examine $\lim _{\beta \uparrow 1} \gamma_{\beta}^{\beta}\left(1, x^{\beta}, y^{\beta}\right)$, which equals $\lim _{\beta \uparrow 1} \gamma_{\beta}^{k}\left(1, x^{\beta}, y^{1}\right)$ since $y^{\beta}=y^{1}$ for all $\beta \in[0,1)$. By lemma 2.2.5 and lemma 2.2.6 there are $\mu_{1}^{1}, \mu_{1}^{2}, \mu_{1}^{3} \in[0,1]$ such that $\sum_{h=1}^{3} \mu_{1}^{h}=1$ and $V_{1}^{k}=\sum_{k=1}^{3} \mu_{1}^{h} \gamma^{k h}\left(x^{1}, y^{1}\right)$ for $k=1,2$. Furthermore those lemmas state that, if under $\left(x^{\beta}, y^{1}\right)$ there are no transitions possible from state 1 to $S^{h}, h \in\{1,2,3\}$, then $\mu_{1}^{h}=0$. For this example we have that $\gamma^{21}\left(x^{1}, y^{1}\right)=1 / 2<3 / 3=V^{21}$. Recall that $\gamma^{21}\left(x^{1}, y^{1}\right)$ and $V^{21}$ are respectively the worth of $\gamma^{2}\left(x^{1}, y^{1}\right)$ and $V^{2}$ for ergodic set $S^{1}=\{1\}$. Hence it follows that $\mu_{1}<1$. This implies, as will be worked out below in a more general setting:

$$
\sum_{t=2}^{3} p\left(t \mid 1,(0,1), y^{1}\right)>0 \text { and } \sum_{t=1}^{3} p\left(t \mid 1,(0,1), y^{1}\right) V_{t}^{2} \geqslant V_{1}^{2}=V^{21}
$$


Since $\gamma^{22}\left(x^{1}, y^{1}\right) \geqslant V^{22}$ and $\gamma^{23}\left(x^{1}, y^{1}\right) \geqslant V^{23}$ we conclude that if, against $y^{1}$, player 1 uses the pure stationary strategy $(0,1)$ in state 1 and $x^{1}$ in $S^{2}$ and $S^{3}$, then with probability 1 a transition to $S^{2} \cup S^{3}$ will occur and hence $\gamma^{2}\left(1, x^{*}, y^{1}\right) \geqslant V_{i}^{2}$, where $x^{*}$ denotes this strategy $(0,1)$ for player 1 (cf. example 1.8.6).

By lemma 1.6 .4 (b) we also have that $\sum_{t=1}^{3} p\left(t \mid 1, x^{*}, y^{1}\right) V_{t}^{1}=V_{1}^{1}$, which follows by taking limits for $\beta$ to 1 in

$\gamma_{\beta}^{1}\left(1, x^{\beta}, y^{1}\right)=\gamma_{\beta}^{1}\left(1, x^{*}, y^{1}\right)=(1-\beta) r^{1}\left(1, x^{*}, y^{1}\right)+\beta \sum_{t=1}^{3} p\left(t \mid 1, x^{*}, y^{1}\right) \gamma_{\beta}^{1}\left(t, x^{*}, y^{1}\right)$.

Since $\gamma^{12}\left(x^{1}, y^{1}\right) \geqslant V^{12}$ and $\gamma^{13}\left(x^{1}, y^{1}\right) \geqslant V^{13}$ we can also conclude that $\gamma^{1}\left(1, x^{*}, y^{1}\right) \geq V 1=V^{11}$ (cf. example 1.8.6).

Hence we have that $\gamma^{k}\left(s, x^{*}, y^{1}\right) \geqslant V_{s}^{k}$ for all $s \in S$ and for $k=1,2$. So both players should be rather satisfied with these limiting average rewards. By lemma 1.5.5 and lemma 1.6.4 we even have that $\gamma^{7}\left(1, x^{*}, y^{1}\right)=\gamma^{1}\left(1, x^{1}, y^{1}\right)$ hence player 1 has no profitable deviations against $y^{1}$. Unfortunately $y^{1}$ is not a best reply for player 2 against $x^{*}$. Hence $\left(x^{*}, y^{3}\right)$ is not a limiting average equilibrium. However it should be observed that if player $\mathbb{1}$ uses the stationary strategy $x^{\lambda}:=(1-\lambda) x^{1}+\lambda x^{*}(=(1-\lambda, \lambda)$ for this example) then, for any $\lambda \in(0,1]$ we still have that for $\left(x^{\lambda}, y^{1}\right)$ a transition from state 1 to $S^{2} \cup S^{3}$ will occur with probability 1 . Moreover $\gamma^{k}\left(s, x^{\lambda}, y^{1}\right)=\gamma^{k}\left(s, x^{*}, y^{1}\right)$ for all $s \in S$, all $\lambda \in(0,1)$ and $k=1,2$.

Now we can construct an almost stationary limiting average $\epsilon$-equilibrium.

Let $c>0$ and let $Y^{(n)}$ and $y^{(n)}$ be as in the proof of theorem 2.3.4. Let $X^{(n)}$ be the random variable denoting the action frequencies of player $\mathbb{1}$ within Car $\left(x^{1}\right)$ up to stage $n$; let $x^{(n)}$ be a realization of $X^{(n)}$. Then, pretending that absorption does not take place, for each $\alpha>0$ and $\delta>0$ there is $N_{a \delta} \in \mathbb{N}$ such that:

$\operatorname{Prob}_{x^{2}, y^{4}}\left\{\left\|X^{(m)}-x^{1}\right\|>\alpha\right.$ for some $\left.n \geqslant N_{\mathrm{a} \delta}\right\}<\delta$ and

$\operatorname{Prob}_{x^{\wedge}, y^{\prime}}\left\{\left\|Y^{(n)}-y^{1}\right\|>\alpha\right.$ for some $\left.n \geq N_{\alpha \delta}\right\}<\delta$.

Choose $\alpha \in(0, \epsilon / 4 M)$ and $\delta>0$ such that:

$(1-\delta)^{4}\left(\gamma^{k}\left(x^{*}, y^{1}\right)-\alpha M\right)-\left(1-(1-\delta)^{4}\right) M \geqslant \gamma^{k}\left(x^{1}, y^{1}\right)-\epsilon / 2$ for $k=1,2$.

Choose $\lambda \in(0, \epsilon / 4 M)$ such that:

Prob $_{x^{\lambda}, y^{\prime}}$ (absorption before stage $\left.N_{\alpha \delta}\right\}<\delta$.

Choose $N_{\lambda} \in \mathbb{N}, N_{\lambda}>N_{a \delta}$ such that:

Prob $_{x^{\lambda}, y^{\prime}}$ \{absorption before stage $\left.N_{\lambda}\right\} \geqslant 1-\delta$.

Define $\pi_{*}^{*}$ by:

a) use $x^{\lambda}$ unless:

i) player 2 chooses $j \notin \operatorname{Car}\left(y^{1}\right)$

ii) $\left\|y^{(n)}-y^{1}\right\|>\alpha$ for some $n \geqslant N_{\text {a } \delta}$ 
iii) at stage $N_{\mathrm{\lambda}}$ play is still in the initial state

b) iff (i), (ii) or (iii) occurs, then use some retaliation strategy $\pi_{v / 2}^{r}$.

Define $\sigma_{\epsilon}^{*}$ analogously.

Now it can be verified that $\left(\pi_{c}^{*}, \sigma_{c}^{*}\right)$ is an almost stationary limiting average equilibrium.

In order to generalize these idleas we introduce the following notations.

\subsubsection{DEFINITION}

For a non-empty $A \subset S, A \neq S$, a pair of stationary strategies $(x, y)$ and $\beta \in[0,1)$ we define:

a) $P(x, y)^{A}$ is the restriction of $P(x, y)$ to rows and columns corresponding with A.

b) $P(x, y)^{A C}$ is the restriction of $P(x, y)$ to rows corresponding with $A$ and columns corresponding with $A^{c}=S \backslash A$.

c) For any $\alpha \in \mathbb{R}^{T}$ the restriction of $\alpha$ to coordinates corresponding with $A\left(A^{c}\right)$ is denoted as $\alpha^{A}\left(\alpha^{4 C}\right)$.

d) $I^{A}$ is the identity matrix of size $|A| \times|A|$.

e) $M_{A}^{\beta}:=\left[m_{3 t}^{\beta}\right]_{s \in A_{t} \in A}:=(1-\beta)\left(I^{A}-\beta P\left(x^{\beta}, y^{1} y^{A}\right)^{-1}\right.$ and $M_{A}^{1}:=\lim _{B+} M_{A}^{B}$, which limit we assume to exist without lass of generality.

f) $N_{A}^{\beta}:=\left[m_{s t}^{\beta,}\right]_{s \in A_{A} \in A^{r}:}=\beta\left(I^{A}-\beta P\left(x^{\beta}, y^{1}\right)^{A}\right)^{-1} P\left(x^{\beta}, y^{1}\right)^{A C}$ and $N_{A}^{1}:=\lim _{\beta \uparrow 1} N_{A}^{B}$, which limit we may also assume to exist.

Using this definition the next lemma follows from elementary calculations. We therefore omit the proof.

\subsubsection{LEMMA}

Notations as above. Then:

a) $m_{s t}^{\beta} \geqslant 0$ for all $s \in A, t \in A$ and $\beta \in[0,1)$;

$n_{s !}^{\beta} \geq 0$ for all $s \in A, t \in A^{i c}$ and $\beta \in[0,1)$.

b) $\sum_{t \in A}^{s} m_{s t}^{\beta}+\sum_{s \in A^{t}} n_{s t}^{\beta}=1$ for all $s \in A$ and $\beta \in[0,1)$.

c) $\gamma_{\beta}^{2}\left(x^{\beta}, y^{1}\right)^{A}=M_{A}^{\beta} r^{2}\left(x^{\beta}, y^{1}\right)^{A}+N_{A}^{\beta} \gamma_{\beta}^{2}\left(x^{\beta}, y^{1}\right)$ for all $\beta \in[0,1)$.

d) $V^{2 A}=M_{A}^{1} r^{2}\left(x^{1}, y^{1}\right)^{A}+N_{A}^{1} V^{2 A C}$.

e) $n_{s i}^{\beta}=\frac{\beta}{1-\beta} \sum_{s \in A} \sum_{i=1}^{m_{s}^{*}} m_{s, s^{*}}^{\beta} x_{s}^{\beta_{s}}\left(i^{*}\right) p\left(t \mid s^{*}, i^{*}, y_{s}^{n^{*}}\right)$ for each $s \in A, t \in A^{c}$.

\subsubsection{LEMMA}

Notations as above.

Let $A=S^{h^{\prime}} \cup S^{h^{2}} \cup \ldots \cup S^{h^{\circ}}$ and let $s \in A$. Then there is $\mu_{s} \in \Delta^{a}$ such that:

$\sum_{t \in A} m_{s t}^{1} r^{2}\left(t, x_{i}^{1}, y_{t}^{\|}\right)=\lambda_{s} \sum_{\alpha=1}^{\Delta} \mu_{s}^{h^{*}} \gamma^{2 t^{*}}\left(x^{1}, y^{1}\right)$, where $\lambda_{s}=\sum_{t \in A} m_{s t}^{1} \in[0,1]$.

\section{ProOF:}

By definition 3.2 .5 we have that $M_{A}^{\beta}\left(I^{A}-\beta P\left(x^{\beta}, y^{1}\right)^{A}\right)=(1-\beta) I^{A}$ for all 
$\beta \in[0,1)$. Hence $M_{A}^{\mathbb{1}}=M_{A}^{\mathbb{1}} P\left(x^{1}, y^{1}\right)^{A}$, which implies that for each $s \in A$ the $s$-th row of $M_{A}^{1}$ is a multiple $\lambda_{s}$ of a stationary distribution for $P\left(x^{1}, y^{1}\right)^{A}$.

Remember that $q^{h^{a} A}$ is the restriction of the unique stationary distribution $q^{h^{a}}$ of $P\left(x^{l}, y^{l}\right)$ on $S^{h^{a}}$ to coordinates corresponding with states in $A$ (cf. definition 2.2.1). So we have that for some $\mu_{s} \in \Delta^{a}$ :

$$
\begin{aligned}
\sum_{i \in A} m_{s l}^{1} r^{2}\left(t, x_{i}^{1}, y_{l}^{1}\right) & =\sum_{t \in A} \lambda_{s} \sum_{a=1}^{u} \mu_{s}^{h^{*}} q_{t}^{h^{a} A} r^{2}\left(t, x_{i}^{1}, y_{s}^{1}\right) \\
& =\lambda_{s} \sum_{a=1}^{u} \mu_{s}^{h^{*}} \sum_{i \in A} q_{t}^{k^{*} A} r^{2}\left(t, x_{b}^{1}, y_{l}^{1}\right) \\
& =\lambda_{s} \sum_{a=1}^{b} \mu_{s}^{h^{*}} \gamma^{2 h^{*}}\left(x^{1}, y^{1}\right)
\end{aligned}
$$

and

$\sum_{t \in A} m_{s t}^{l}=\sum_{t \in A} \lambda_{s} \sum_{\alpha=1}^{a} \mu_{s}^{h^{a}} q_{t}^{h^{a} A}=\lambda_{s} \sum_{\alpha=1}^{a} \mu_{s}^{h^{a}} \sum_{t \in A} q_{t}^{h^{\alpha} A}=\lambda_{s} \sum_{\alpha=1}^{a} \mu_{s}^{h^{a}}=\lambda_{s} \in[0,1]$

by lemma 3.2 .6 (a) and (b).

\subsubsection{LEMMA}

Notations as above.

For $s^{*} \in A$ and $i^{*} \in \operatorname{Car}\left(x_{s}^{\beta}\right)$ with $\sum_{t \in A^{*}} p\left(t \mid s^{*}, i^{*}, y_{s}^{\mathbb{L}_{*}}\right)>0$ define:

$\lambda_{s}\left(s^{*}, i^{*}\right):=\lim _{\beta \uparrow 1} \frac{\beta}{1-\beta} m_{s s}^{\beta} \cdot x_{s}^{\beta}\left(i^{*}\right) \sum_{i \in A^{*}} p\left(t \mid s^{*}, i^{*}, y_{s}^{1}\right)$.

Suppose that there is at least one pair $\left(s^{*}, i^{*}\right)$ with this property. Then:

$\sum_{i \in A^{*}} n_{s t}^{1} V_{t}^{2}=\sum_{s^{*}, i^{*}} \lambda_{s}\left(s^{*}, i^{*}\right) \sum_{t \in A^{*}}\left[\frac{p\left(t \mid s^{*}, i^{*}, y_{s}^{1}\right) V_{t}^{2}}{\sum_{i \in A^{*}} p\left(t^{*} \mid s^{*}, i^{*}, y_{s}^{1}\right)}\right]$ and
$\sum_{t \in A^{*}} n_{s t}^{1}=\sum_{s^{*}, t^{*}} \lambda_{s}\left(s^{*}, i^{*}\right)=1-\lambda_{s}$

Proof:

By Iemma 3.2.6 (e): $n_{s t}^{\beta}=\frac{\beta}{1-\beta} \sum_{s^{*} \in A} \sum_{i}^{m_{i}^{*}=\mathbb{1}} m_{s s}^{\beta} \cdot x_{s}^{R}\left(i^{*}\right) p\left(t \mid s^{*}, i^{*}, y^{l^{*}}\right)$.

Hence $n_{s i}^{1}=\lim _{\beta \uparrow 1} \frac{\beta}{1-\beta} \sum_{s \in A} \sum_{i=1}^{m_{s}^{*}} m_{s s}^{\beta} \cdot x_{s}^{A}\left(i^{*}\right) p\left(t \mid s^{*}, i^{*}, y_{s}^{\prime}\right)$

$$
=\sum_{s, i} \lambda_{s}\left(s^{*}, i^{*}\right) \frac{p\left(t \mid s^{*}, i^{*}, y_{s}^{1 \cdot}\right)}{\sum_{i \in A^{*}} p\left(t^{*} \mid s^{*}, i^{*}, y_{s}^{1 \cdot}\right)}
$$

for $s \in A$ and $t \in A^{t}$, which proves this lemma (cf. lemma 3.2 .6 (b) and lemma 3.2.7).

\subsubsection{LEMMA}

Let $A=\bigcup_{\alpha=1}^{a} S^{h^{\alpha}}$ and let $\varnothing \neq A \neq S$. Suppase $V_{s}^{2}=V_{t}^{2}=: V_{A}^{2}$ for all $s, t \in A$. 
If $V_{A}^{2}>\gamma^{2 h^{*}}\left(x^{1}, y^{1}\right)$ for $\alpha=1,2, \ldots, a$, then there is $s^{*} \in A$ and $i^{*} \in \operatorname{Car}\left(x_{s}^{\beta}\right)$ such that: $\sum_{i \in A^{*}} p\left(t \mid s^{*}, i^{*}, y_{s}^{1}\right)>D$ and $\sum_{i \in S} p\left(t \mid s^{*}, i^{*}, y_{s}^{l_{*}}\right) V_{t}^{2} \geq V_{A}^{2}=V_{s}^{2}$.

Furthermore: $\sum_{t \in S} p\left(t \mid s^{*}, i^{*}{ }^{*} y_{s}{ }^{l_{*}}\right) V_{t}^{1}=\mathscr{V}_{s}^{1}$.

Proof:

Take $s \in S$. By lemma 3.2 .6 (d): $V_{s}^{2}=\sum_{t \in A} m_{s t}^{1} r^{2}\left(t, x_{t}^{1}, y_{t}^{1}\right)+\sum_{t \in A^{*}} n_{s t}^{1} V_{s}^{2}$. Applying lemmas 3.2 .7 and 3.2.8 we derive:

$$
V_{s}^{2}=\lambda_{s} \sum_{a=1}^{a} \mu_{s}^{h^{s}} \gamma_{s}^{h^{*}}\left(x^{1}, y^{1}\right)+\sum_{s^{*}, i^{*}} \lambda_{s}\left(s^{*}, i^{*}\right) \sum_{t \in A^{*}} \frac{p\left(t \mid s^{*}, i^{*}, y_{s}{ }^{*}\right) V_{t}^{2}}{\sum_{t \in A^{*}} p\left(t^{*} \mid s^{*}, i^{*}, y_{s}{ }^{*}\right)}
$$

for certain $\lambda_{s} \in[0,1], \mu_{s} \in \Delta^{a}$ and $\lambda_{s}\left(s^{*}, i^{*}\right) \in[0,1]$, and it holds that:

$$
\lambda_{s}+\sum_{s, i} \lambda_{s}\left(s^{*}, i^{*}\right)=1 \text {. }
$$

Since $\gamma^{2 h^{a}}\left(x^{1}, y^{1}\right)<V_{s}^{2}$ for all $\alpha \in\{1,2, \ldots, a\}$, we conclude that $\lambda_{s}<1$ and for at least one $\left(s^{*}, i^{*}\right)$ with $\lambda_{s}\left(s^{*}, i^{*}\right)>0$ we have:

$$
\sum_{i \in A^{*}} p\left(t \mid s^{*}, i^{*}, y^{1}\right)>0 \text { and } \sum_{t \in A^{*}} \frac{p\left(t \mid s^{*}, i^{*}, y_{s}^{\mathrm{l}}\right) V_{t}^{y^{2}}}{\sum_{i \in A^{*}} p\left(t^{*} \mid s^{*}, i^{*}, y_{s}^{*}\right)} \geqslant V_{s}^{2}=V_{A^{*}}^{2}
$$

Since $V_{t}^{2}=V_{A}^{2}$ for all $t \in A$, it follows that $\sum_{t \in S} p\left(t \mid s^{*}, i^{*}, y_{s}^{1}\right) V_{t}^{2} \geqslant V_{A}^{2}$.

By lemma 2.3.3 we also have $\sum_{t \in S} p\left(t \mid s^{*}, i^{*}, y_{s}^{1} \in V^{*}\right) V_{t}^{1}=V_{s}^{1 .}$.

The following lemma can be proved analogously.

\subsubsection{LEMMA}

Let $A=\bigcup^{a} S^{h^{*}}$ and let $\varnothing \neq A \neq S$. Suppose $V_{s}^{1}=V_{t}^{1}=: V_{A}^{1}$ for all $s, t \in A$.

If $V_{A}^{1}>\gamma^{1 h^{k}}\left(x^{1}, y^{1}\right)$ for $\alpha=1,2, \ldots$, a, then there is $s^{*} \in A$ and $j^{*} \in \operatorname{Car}\left(y_{s}^{\beta}\right)$

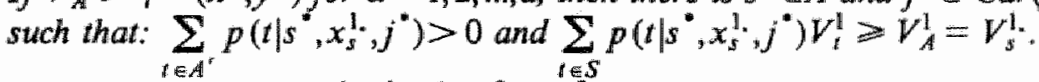

Furthermore: $\sum_{t \in S^{*}} p\left(t \mid s^{*}, x_{s}^{1}, j^{*}\right) V_{t}^{2}={\stackrel{i \in S}{V_{s}^{2}}}^{2}$.

Observe that in the above lemmas $i^{*} \in \operatorname{Car}\left(x_{s}^{\beta}\right) \backslash \operatorname{Car}\left(x_{s}^{1}\right)$ and

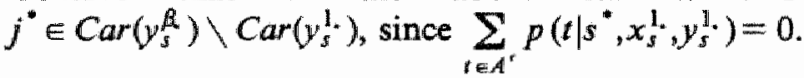

\subsubsection{THEOREM}

Let $\left(x^{*}, y^{*}\right)$ be a pair of stationary strategies with the following properties.

a) For each $h \in I_{1}$ there is $s^{h} \in S^{h}$ and $j^{h} \in \operatorname{Car}\left(y_{s}^{B_{n}}\right)$ with $y_{s^{*}}=j^{h}$ such that: $\sum_{t \notin s^{*}} p\left(t \mid s^{h}, x_{s^{h}}^{l^{h}}, j^{h}\right)>0$, 


$$
\begin{aligned}
& \sum_{t \in S} p\left(t \mid s^{h}, x_{s}^{1^{2}}, j^{h}\right) V_{t}^{2}=V_{s}^{2} \text { and } \sum_{t \in S} p\left(t \mid s^{h}, x_{s^{h}}^{h^{h}}, j^{h}\right) V_{t}^{1} \geqslant V_{s^{1}}^{l_{k}}=V^{1 h} \text {. } \\
& \text { Also } y_{s}^{*}=y_{s}^{1} \text { for all } s \in S \backslash \bigcup_{h \in l}\left\{s^{h}\right\} \text {. }
\end{aligned}
$$

b) For each $h \in I_{2}$ there is $s^{h} \in S^{h}$ and $i^{h} \in \operatorname{Car}\left(x_{s}^{\beta}\right)$ with $x_{s^{*}}^{*}=i^{h}$ such that:

$$
\begin{aligned}
& \sum_{t \notin s^{4 h}} p\left(t \mid s^{h}, i^{h}, y_{s^{k}}^{l^{k}}\right)>0
\end{aligned}
$$

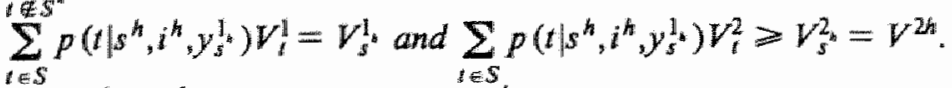

$$
\begin{aligned}
& \text { Also } x_{s}^{*}=x_{s}^{1} \text { for all } s \in S \backslash \bigcup_{h \in J_{2}}\left\{s^{h}\right\} \text {. }
\end{aligned}
$$

c) Each $s \in S^{\prime} \backslash S^{\prime}$ is transient with respect to $\left(x^{*}, y^{*}\right)$.

Then $P\left(x^{*}, y^{*}\right) V^{k} \geqslant V^{k}$ and $\gamma^{k}\left(x^{*}, y^{*}\right) \geqslant V^{k}$ for $k=1,2$.

\section{Proof:}

By lemma 2.3.3 and by (a) and (b) it follows that $P\left(x^{*}, y^{*}\right) V^{k} \geqslant V^{k}$, which also implies that $Q\left(x^{*}, y^{*}\right) V^{k} \geqslant V^{k}$. For $s \in S^{I}$ we have by definition that $\gamma^{k}\left(s, x^{*}, y^{*}\right)=\gamma^{k}\left(s, x^{1}, y^{1}\right) \geqslant V^{k}$.

Since all states in $S \backslash S^{I}$ are transient with respect to $\left(x^{*}, y^{*}\right)$ it holds that (cf. lemma 1.5 .2 (e) and lemma 1.5.5):

$$
\begin{aligned}
\gamma^{k}\left(x^{*}, y^{*}\right) & =Q\left(x^{*}, y^{*}\right) r^{k}\left(x^{*}, y^{*}\right)=Q\left(x^{*}, y^{*}\right) Q\left(x^{*}, y^{*}\right) r^{k}\left(x^{*}, y^{*}\right)= \\
& =Q\left(x^{*}, y^{*}\right) \gamma^{k}\left(x^{*}, y^{*}\right) \geqslant Q\left(x^{*}, y^{*}\right) V^{k} \geqslant V^{k} .
\end{aligned}
$$

\subsubsection{LEMMA}

For $\left(x^{*}, y^{*}\right)$ as in theorem 3.2 .11 and $\lambda \in(0,1)$ define:

$x_{s}^{\lambda}:=(1-\lambda) x_{s}^{1}+\lambda x_{s}^{*}$ and $y_{s}^{\lambda}:=(1-\lambda) y_{s}^{1}+\lambda y_{s}^{*}$ for all $s \in S$.

Then the following statements hold:

a) Each $s \in S^{\prime}$ is recurrent with respect to $\left(x^{\lambda}, y^{\lambda}\right)$ and each $s \in S \backslash S^{\prime}$ is transient with respect to $\left(x^{\lambda}, y^{\lambda}\right)$.

b) $P\left(x^{\lambda}, y^{\lambda}\right) V^{k} \geqslant V^{k}$ and $\gamma^{k}\left(x^{\lambda}, y^{\lambda}\right) \geqslant V^{k}$ for $k=1,2$.

\section{Proof:}

For all $s \in S \backslash \bigcup_{h \in L}\left(s^{h}\right\}$ we have $\left(x_{s}^{\lambda}, y_{s}^{\lambda}\right)=\left(x_{s}^{*}, y_{s}^{*}\right)$ and hence it follows that $p\left(t \mid s, x_{s}^{\lambda}, y_{s}^{\lambda}\right)=p\left(t \mid s, x_{s}^{*}, y_{s}^{*}\right)$ for those $s$ and for all $t \in S$.

For $s \in S^{h}, h \in I_{1} \cup I_{2}$, we have that $p\left(t \mid s_{s}, x_{s}^{\lambda}, y_{s}^{\lambda}\right)=\lambda p\left(t \mid s, x_{s}^{*}, y_{s}^{*}\right)$ for all $t \notin S^{h}$.

Since $\lambda>0$ the ergodic sets for $\left(x^{\lambda}, y^{\lambda}\right)$ are precisely the same as those for $\left(x^{*}, y^{*}\right)$. By lemma 2.3 .3 and theorem 3.2 .11 we have that $P\left(x^{\lambda}, y^{\lambda}\right) V^{k} \geqslant V^{k}$ for $k=1,2$. Using arguments as in the proof of theorem 3.2.11 we derive that $\gamma^{k}\left(x^{\lambda}, y^{\lambda}\right) \geqslant V^{k}$ for $k=1,2$.

Now we can formulate the main theorem of this section.

\subsubsection{THEOREM}

Let $\left(x^{\lambda}, y^{\lambda}\right)$ be as in lemma 3.2.12.

If $p\left(t \mid s, x_{s}^{\lambda}, y_{s}^{\lambda}\right)=0$ for all $s \in T$ and $t \in S \backslash\left(S^{I_{0}} \cup T\right)$, 
then $\left(x^{\lambda}, y^{\lambda}\right)$ can be supplemented with suitable retaliation threats to achieve an almast stationary exequilibritum, for $\lambda \in(0,1)$ sufficiently small.

\section{Proof:}

Let $>0$. For each $\eta \in(0,1)$ there is $N_{\eta} \in N$ such that, with probability at least $1-\eta$, the expected number of transitions among elements of the set $\left(S^{h}: h \in I_{1} \cup I_{2}\right\} \cup T$ will be at most $N_{\eta}$ with respect to $\left(x^{\lambda}, y^{\lambda}\right)$ for any initial state $s \in S \backslash S^{l}$. Furthermore $N_{\eta}$ does not depend on $\lambda \in(0,1)$.

Now choose $\eta, \delta \in(0,1)$ such that for all $s \in S \backslash S^{l}$ :

$$
(1-\eta)(1-\delta)^{4}\left(\gamma^{k}\left(s, x^{\lambda}, y^{\lambda}\right)-\epsilon / 4\right)-\left(1-(1-\eta)(1-\delta)^{4}\right) M \geqslant \gamma^{k}\left(s, x^{\lambda}, y^{\lambda}\right)-\epsilon / 2
$$

Next choose $\alpha \in\left(0, \epsilon / 4 M N_{\eta}\right)$ and $N_{\alpha \delta} \in \mathbb{N}$ such that

$\operatorname{Prob}_{x^{\lambda}, y^{*}}\left\{\left\|Y_{s}^{(n)}-y_{s}^{1}\right\|>\alpha\right.$ for some $n \geqslant N_{a \delta}$ and some $\left.s \in S\right\}<\delta$ and

$\operatorname{Prob}_{x^{2}, y^{*}}\left\{\left\|X_{s}^{(n)}-x_{s}^{1}\right\|>\alpha\right.$ for some $n \geqslant N_{a \delta}$ and some $\left.s \in S\right\}<\delta$.

Here $Y_{s}^{(n)}\left(X_{s}^{(n)}\right)$ should be interpreted as the random variable denoting the action frequencies for player 2 (1) after $n$ stages in state $s$.

Observe that $N_{\alpha \delta}$ is independent of the initial state.

Choose $\lambda \in(0, \epsilon / 4 M)$ and $N_{\lambda} \geqslant N_{\text {of }}$ such that:

Prob $_{x^{k}, y^{*}}$ \{transition from $S^{h}$ to $S \backslash S^{h}$ within $N_{a \delta}$ stages, with $\left.h \in I_{1} \cup I_{2}\right\}<\delta$, $\operatorname{Prob}_{x^{x}, y^{\lambda}}$, transition from $S^{h}$ to $S \backslash S^{h}$ within $N_{\lambda}$ stages, with $h \in I_{1} \cup I_{2}$ \} $\geqslant(1-\delta)^{1 / N}$.

Define strategy $\pi_{i}^{*}$ for player 1 by:

a) use strategy $x^{\lambda}$ unless (i), (ii) or (iii) below occurs:

i) for some $s \in S$ and some $n \geqslant N_{\alpha \delta}:\left\|y_{s}^{(n)}-y_{s}^{1}\right\|>\alpha$, where $y_{s}^{(n)}$ is a realization of $Y_{s}^{(n)}$.

ii) player 2 chooses an action outside $\operatorname{Car}^{z}\left(y^{\lambda}\right)$.

iii) after $N_{\eta}$ transitions among elements of $\left\{S^{h}: h \in I_{1} \cup I_{2}\right\} \cup T$, the play is still in $S \backslash S^{l}$.

iv) play is in the same $S^{h}, h \in I_{1} \cup I_{2}$ for more than $N_{\lambda}$ stages.

b) if (i), (ii), (iii) or (iv) occurs, then use some retaliation strategy $\pi_{\epsilon / 4}^{r}$, from that moment on.

For player 2 the strategy $\sigma_{c}^{*}$ is defined analogously.

If the players use $\left(\pi_{\tilde{\varepsilon}}^{*}, \sigma_{t}^{*}\right)$ then with probability at most $\left(1-(1-\eta)(1-\delta)^{4}\right)$ some player may start using his retaliation strategy and with probability at least $(1-\eta)(1-\delta)^{4}$ the players remain using $\left(x^{\lambda}, y^{\lambda}\right)$ forever.

Hence, by choice of $\eta, \alpha$ and $\delta$, we have $\gamma^{k}\left(\pi_{e}^{*}, \sigma_{k}^{*}\right) \geq \gamma^{k}\left(x^{\lambda}, y^{\lambda}\right)-\epsilon / 2$ for $k=1,2$.

Now suppose player 1 uses $\pi_{i}^{*}$ and player 2 uses some arbitrary $\sigma$.

If player 1 detects a deviation at stage $n$, where player 2's action was $j$ in state $s$, then with probability at least $(1-\lambda)$ player $\mathbb{1}$ was using $x_{s}^{j}$ at that stage and with probability at most $\lambda$ player 1 was using some $i^{h}$, where $s=s^{h}$. Hence by lemma 2.3.3, player 1's retaliation in that case gives that the limiting average reward will be at most $(1-\lambda)\left(V_{s}^{2}+\epsilon / 4\right)+\lambda M \leqslant V_{s}^{2}+\epsilon / 2$ $\leqslant \gamma^{k}\left(s, x^{\lambda}, y^{\lambda}\right)+\epsilon / 2$.

If player 1 does not detect any deviation then at each transition among 
elements of $\left\{S^{h}: h \in I_{1} \cup I_{2}\right\} \cup T$ player 2 can gain at most $2 \alpha M \leqslant \epsilon / 2 N_{\text {m. }}$. Since there are at most $N_{\eta}$ transitions without player 1 starting retaliation, we find that the limiting average reward to player 2 will be at most $\gamma^{k}\left(x^{\lambda}, y^{\lambda}\right)+\epsilon / 2$.

Here it should be noticed that we use the condition of the theorem to conclude that player 2 cannot deviate in $T$ (and neither can player 1). This is due to the following argument.

If player 2 chooses an action outside $\operatorname{Car}\left(y^{\lambda}\right)$ in $T$, then player 1 will retaliate directly according to the definition of $\pi_{\varepsilon}^{*}$. Retaliation is possible because by lemma 2.3 .3 we have $P\left(x^{1}, y\right) V^{2} \leqslant V^{2}$ for all stationary strategies $y$. By lemma 1.6 .2 it is sufficient to consider deviations by stationary strategies.

Now suppose that the play is in $T$ and player 2 uses a stationary strategy $y$ with $\operatorname{Car}\left(y_{s}\right) \subset \operatorname{Car}\left(y_{s}^{x}\right)$ for all $s \in T$ and $y_{s}=y_{s}^{1}$ for $s \in S^{I_{0}}$.

We denote restrictions of $r^{2}(),, r^{2}(),, P(),, Q($,$) to coordinates,$ respectively rows and columns, corresponding with $T \cup S^{I_{0}}$ by $\vec{r}($,$) ,$ $\vec{\gamma}(),, \bar{P}(),, \bar{Q}($,$) . If the condition of this theorem holds, then any play$ which is in $T$ at some stage, will remain in $T \cup S^{l_{0}}$ forever, in case the players use $\left(x^{1}, y\right)$ from that stage on. If some state $t \in T$ is recurrent with respect to $\left(x^{1}, y\right)$, then player 1 using $\pi_{\varepsilon}^{*}$ would start, with probability 1 , to retaliate player 2. By lemma 2.3.3 this would give player 2 a limiting average reward of at most $V_{t}^{2}+\epsilon / 2$. On the other hand, if all states in $T$ are transient with respect to $\left(x^{1}, y\right)$ then we have by lemma 2.3.3:

$$
\begin{aligned}
\bar{\gamma}^{2}\left(x^{1}, y\right) & =\bar{Q}\left(x^{1}, y\right) \bar{r}^{2}\left(x^{1}, y\right)=\bar{Q}\left(x^{1}, y\right) \bar{Q}\left(x^{1}, y\right) \bar{r}^{2}\left(x^{1}, y\right) \\
& =\bar{Q}\left(x^{1}, y\right) \bar{Q}\left(x^{1}, y^{1}\right) \bar{r}^{2}\left(x^{1}, y^{1}\right) \\
& =\bar{Q}\left(x^{1}, y\right) \bar{V}^{2} \\
& \leqslant \bar{V}^{2} .
\end{aligned}
$$

Since we know that, once the play is in $S^{I}$ neither player 1 nor player 2 can gain more that $c$ by deviating from $\left(\pi_{,}^{*}, \sigma_{\varepsilon}^{*}\right)$, the above implies that against $\pi_{\varepsilon}^{*}$ player 1 has no profitable deviations while the play is in $T$.

\subsubsection{REMARK}

If for any converging sequence of stationary $\beta$-discounted equilibria $\left\{\left(x^{\beta}, y^{\beta}\right) ; \beta \in[0,1)\right\}$ one can choose $s^{h}, i^{h}, j^{h}$, for $h \in I_{1} \cup I_{2}$, such that the conditions of theorem 3.2.13 hold, then $\left(x^{\lambda}, y^{\lambda}\right)$ can be supplemented with retaliation threats to achieve an almast stationary limiting average e-equilibrium for $\lambda$ sufficiently small.

Let us now consider an example to clarify the condition in theorem 3.2.13. 


\subsubsection{EXAMPLE}

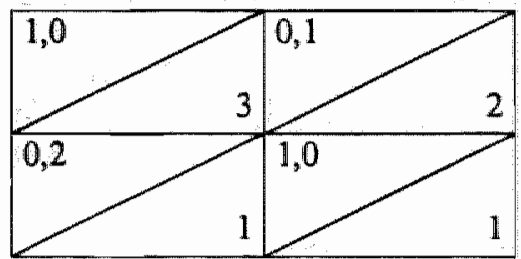

State 1

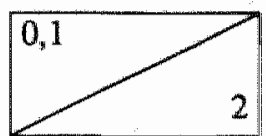

State 2

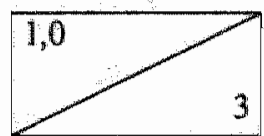

State 3

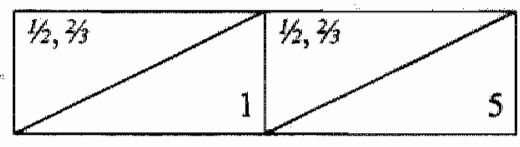

State 4

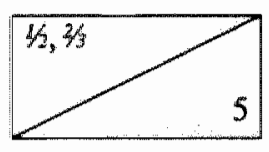

State 5

Observe that we have simply added states 4 and 5 to example 3.2 .3 .

A $\beta$-discounted equilibrium $\left(x^{\beta}, y^{\beta}\right)$ is given by:

$x^{\beta}:=(((2-2 \beta) /(3-2 \beta), 1 /(3-2 \beta)), 1,1,1,1)$ and $y^{\beta}:=((1 / 2,1 / 2), 1,1,(1 / 2,1 / 2), 1)$.

Then $\gamma_{\beta}^{1}\left(x^{\beta}, y^{\beta}\right)=(1 / 2,0,1,1 / 2,1 / 2)$ and $\gamma_{\beta}^{2}\left(x^{\beta}, y^{\beta}\right)=(2 / 3,1,0,2 / 2,2 / 3)$ for the respective initial states. Hence $V^{1}=(1 / 2,0,1,1 / 2,1 / 2)$ and $V^{2}=(2 / 3,1,0,3 / 3,3 / 3)$. Also: $\gamma^{1}\left(x^{1}, y^{1}\right)=(1 / 2,0,1,1 / 2,1 / 2)$ and $\gamma^{2}\left(x^{1}, y^{1}\right)=(1,1,0,5 / 6,2 / 3)$. Hence $I_{1}=I_{2}=\varnothing$, $S^{\prime}=\{1,2,3,5\}, S^{l_{0}}=\{2,3,5\}, T=\{4\}$.

Notice that $p\left(1 \mid 4, x_{4}^{1}, y_{4}^{1}\right)=1 / 2 \neq 0$, so the condition of theorem 3.2 .13 is not fulfilled. Although for initial states in $\{1,2,3,5\}$ the strategies $\left(x^{1}, y^{1}\right)$ can be supplemented to achieve an almost stationary limiting average $\epsilon$-equilibrium (cf. example 3.2.3), this is impossible for initial state 4, since player 2 could gain $1 / 3$ by using $y^{*}=((1 / 2,1 / 2), 1,1,(1,0), 1)$ against $x^{1}$, for initial state 4 . Player 1 cannot check in state 4 whether player 2 is using $y^{*}$ or $y^{1}$.

If however we had started with $y_{4}^{\beta}=(0,1)$, then the condition of theorem 3.2.13 would have been fulfilled. If we had started with $y_{4}^{\beta}=(1,0)$, then the condition of theorem 3.2.13 would not have been fulfilled, but since player 2 has no profitable deviations within $\operatorname{Car}\left(y_{4}^{\beta}\right)$ we could also in this case establish an e-equilibrium by supplementing $\left(x^{1}, y^{1}\right)$ with retaliation threats.

So if the condition of theorem 3.2.13 is not fulfilled, then this does not necessarily mean that it is impossible to achieve a limiting average e-equilibrium from $\left(x^{1}, y^{1}\right)$. All one really needs is that neither player 1 nor player 2 has profitable deviations from $x_{s}^{1}$, respectively $y_{s}^{1}$, in any $s \in T$, that cannot be detected by the opponent (with probability near 1 ). Hence we can make the following remarkable observation:

If after each stage both players were told what mixed actions have been used at that stage, then the condition of theorem 3.2.11 would be sufficient to achieve an almost stationary limiting average c-equilibrium. Any deviation could be detected immediately and hence retaliation threats could be used for 
all states. Notice that for zero-sum stochastic games the limiting average value and limiting average e-optimal strategies are independent of this information.

In the above construction we used just one sequence $\left\{\left(x^{\beta}, y^{\beta}\right): \beta \in[0,1)\right\}$ of stationary $\beta$-discounted equilibria. One could also use an iteration argument: start with an arbitrary sequence $\left\{\left(x^{\beta}, y^{\beta}\right): \beta \in[0,1)\right\}$ of stationary $\beta$-discounted equilibria; find all strong initial states that can be found by the above techniques; replace those initial states $s$ by absorbing states $s$, i.e. each player has just one action in $s$ and $p(s \mid s, 1,1)=1$, with $r^{k}(s, 1,1)=\gamma^{k}\left(s, x^{\lambda}, y^{\lambda}\right)$; in this new stochastic game again take a sequence of stationary $\beta$-discounted equilibria and try to find more strong initial states; repeat this procedure. In certain cases it may lead to an e-equilibrium for all initial states. If however the stationary $\beta$-discounted equilibria are chosen arbitrary at each iterative step, then one not necessarily finds new strong initial states. We give two examples to illustrate these ideas.

Suppose in example 3.2.15 we had indeed started with $\left(x^{\beta}, y^{\beta}\right)=$ $((((2-2 \beta) /(3-2 \beta), 1 /(3-2 \beta)), 1,1,1,1),((1 / 2,1 / 2), 1,1,(1 / 2,1 / 2), 1))$. Then in the first step we would have found the strong initial states $1,2,3$ and 5 . Replacing these by absorbing states gives the following stochastic game.

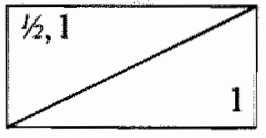

State 1

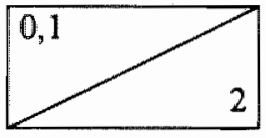

State 2

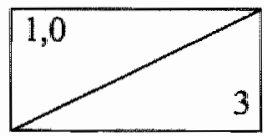

State 3

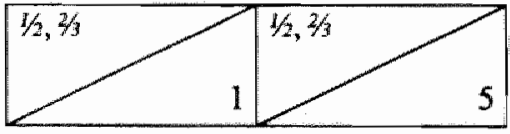

State 4

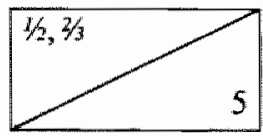

State 5

The unique stationary $\beta$-discounted equilibria for this stochastic game are given by $y_{4}^{\beta}=(1,0)$. So for this new stochastic game $S^{I_{v}}=\{1,2,3,5\}$ and $T=\{4\}$ and the condition of theorem 3.2 .13 is fulfilled; hence we can achieve a limiting average $\epsilon$-equilibrium for this stochastic game. This $\epsilon$-equilibrium induces an 6 -equilibrium for the original stochastic game. 


\subsubsection{EXAMPLE}

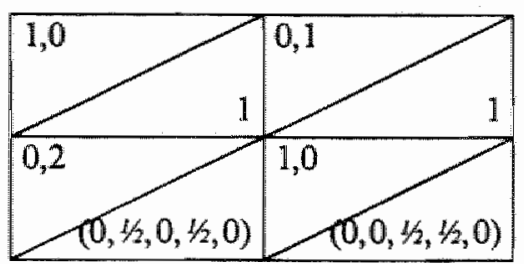

State 1

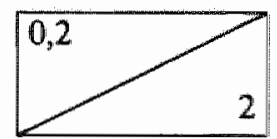

State 2

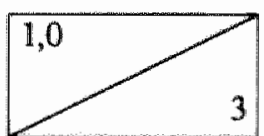

State 3

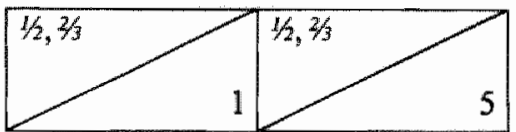

State 4

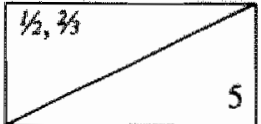

State 5

It can be verified that for this stochastic game stationary $\beta$-discounted equilibria are for instance:

$$
\left(x^{\beta}, y^{\beta}\right)=((((2-\beta) /(3-2 \beta),(1-\beta) /(3-2 \beta)), 1,1,1,1),((1 / 2,1 / 2), 1,1,(1 / 2,1 / 2), 1)) .
$$

We find:

$$
\gamma_{\beta}^{1}\left(x^{\beta}, y^{\beta}\right)=(1 / 2,0,1,1 / 2,1 / 2) \text { and } \gamma_{\beta}^{2}\left(x^{\beta}, y^{\beta}\right)=(3 / 3,2,0,2 / 3,3 / 3) \text { for all } \beta \in[0,1) \text {. }
$$

So $V^{1}=(1 / 2,0,1,1 / 2,1 / 2)$ and $V^{2}=(2 / 3,2,0,3 / 2,2 / 3)$.

Furthermore

$\gamma^{1}\left(x^{1}, y^{1}\right)=(1 / 2,0,1,1 / 2,1 / 2)=V^{1}$ and $\gamma^{2}\left(x^{1}, y^{1}\right)=(1 / 2,2,0,7 / 12,4 / 3) \leqq V^{2}$.

Hence $S^{I_{0}}=S^{l}=\{2,3,5\}, S^{I_{2}}=\{1\}, T=\{4\}$ and $I_{1}=\varnothing$. Clearly the condition of theorem 3.2 .13 is not fulfilled. The strong initial states we find are $\{2,3,5\}$. By the techniques in the proof of theorem 3.2.13 it is not possible to achieve an $\epsilon$-equilibrium for initial state 1.

Iteration does not work either; replacing the strong initial states $\{2,3,5\}$ by absorbing states does not change the stochastic game situation, so in the second step we could again choose $\left(x^{\beta}, y^{\beta}\right)$ the same as above.

Of course, by choosing $y_{4}^{\theta}=(1,0)$ or $(0,1)$ one could establish an c-equilibrium. 



\section{Chapter 4}

\section{Special classes of stochastic games}

\subsection{INTRODUCTION}

In this chapter we discuss the impact of the results from the previous chapters on several special classes of stochastic games. Special classes of stochastic games are stochastic games with an additional property on the payoff and/or the transition structure.

The special classes we consider are: unichain stochastic games (section 4.2), stochastic games with state independent transitions (section 4.3) and repeated games with absorbing states (section 4.4).

\subsection{UNICHAIN STOCHASTIC GAMES}

\subsubsection{DEFINITION}

A unichain stochastic game is a stochastic game with the property that, for any pair of stationary strategies $(x, y)$, there is just one irreducible set of states.

Unichain stochastic games were considered by Gillette [1957] and by Hoffman \& Karp [1966] who proved that in the zero-sum case both players have stationary limiting average optimal strategies. Later Rogers [1969], Sobel [1971] and Federgruen [1978] independently showed that in the general-sum case there exist stationary limiting average equilibria. Those proofs are all based on continuity properties of $\gamma^{k}(x, y)$ on $X \times Y$ and they all use some fixed point theorem. Using results of chapter 2 we give new proofs for these facts.

\subsubsection{THEOREM}

Let $\left\{\left(x^{\beta}, y^{\beta}\right): \beta \in[0,1)\right\}$ be a sequence of stationary $\beta$-discounted equilibria in a general-sum unichain stochastic game and let $\left(x^{\|}, y^{1}\right)=\lim _{\beta \uparrow 1}\left(x^{\beta}, y^{\beta}\right)$.

Then $\left(x^{1}, y^{1}\right)$ is a stationary limiting average equilibrium.

\section{PROOF:}

Since we are dealing with a unichain stochastic game, there is just one ergodic set for any pair of stationary strategies $(x, y)$. Hence each row of $Q(x, y)$ is equal to the unique stationary distribution for the related Markoy chain. In view of lemma 1.5 .5 (b) we conclude that $\gamma^{k}(s, x, y)=\gamma^{k}(t, x, y)$ for all $s, t \in S$ and $k=1,2$. Now suppose player 2 is using $y \in Y$ against $x^{1}$. By lemma 2.2 .6 we have that $\gamma^{2}\left(s, x^{1}, y\right)=\lim _{\beta \uparrow 1} \gamma_{\beta}^{2}\left(s, x^{\beta}, y\right) \leqslant \lim _{\beta \uparrow 1} \gamma_{\beta}^{2}\left(s, x^{\beta}, y^{\beta}\right)=\gamma^{2}\left(s, x^{1}, y^{1}\right)$, for 
all $s \in S$. From lemma 1.6 .2 it now follows that $y^{\prime \prime}$ is a limiting average best reply for player 2 against $x^{1}$. Similarly it can be shown that $x^{7}$ is a limiting average best reply for player 1 against $y^{1}$.

The above theorem implies that for zero-sum unichain stochastic games the limiting average value exists and equals the limit of $\beta$-discounted values; moreover it follows that the limiting average value for initial states $s$ and $t$ is the same for all $s, t \in S$. Furthermore both players have stationary limiting average optimal strategies. This is formulated in the next theorem.

\subsubsection{THEOREM}

For a zero-sum unichain stochastic game the limiting average value $v^{1}$ exists and $v^{1}=\lim _{\beta \uparrow 1} v_{\beta}^{l}$. Furthermore $v_{s}^{1}=v_{l}^{l}$ for all $s, t \in S$.

Let $\left.\left\{x^{\beta}: \beta \in[0,1)\right\} \quad\left(y^{\beta}: \beta \in[0,1)\right\}\right)$ be a sequence of stationary $\beta$-discounted optimal strategies for player 1 (2) and let $x^{1}=\lim _{\beta \uparrow 1} x^{\beta}\left(y^{1}=\lim _{\beta \uparrow 1} y^{\beta}\right)$.

Then $x^{11}\left(y^{1}\right)$ is a stationary limiting average optimal strategy for player 1 (2).

\section{Proof:}

It is easy to verify that for a zero-sum stochastic game a pair of stationary strategies $(x, y)$ is an equilibrium if and only if $x$ is optimal for player 1 and $y$ is optimal for player 2. Since by theorem 4.2 .3 the pair of strategies $\left(x^{1}, y^{1}\right)$ is a limiting average equilibrium and since $\gamma^{\mathbb{1}}\left(x^{1}, y^{1}\right)=\lim _{\beta \uparrow 1} \gamma_{\beta}^{\mathrm{l}}\left(x^{\beta}, y^{\beta}\right)=\lim _{\beta \uparrow 1} v_{\beta}^{\mathbb{1}}$ is independent of the initial state, the proof is complete.

\subsection{STOCHASTIC GAMES WITH STATE INDEPENDENT TRANSITIONS}

\subsubsection{DEFINITION}

A stochastic game with state independent transitions (SIT) is a stochastic game for which there are $m, n \in \mathbb{N}$ such that $m_{s}=m$ and $n_{s}=n$ for all $s \in S$ and for which furthermore $p(s, i, j)=p(t, i, j)$ for all $s, t \in S$ and all $i, j$.

A stochastic game with state independent transitions and separable rewards (SER-SIT) is a SIT stochastic game with the additional property that there are $c^{k}: S \rightarrow \mathbb{R}$ and $a^{k}:\{1,2, \ldots, m\} \times\{1,2, \ldots, n\} \rightarrow \mathbb{R}$, for $k=1,2$ such that $r^{k}(s, i, j)=c^{k}(s)+a^{k}(i, j)$ for all $s, i, j$ and $k=1,2$.

An early appearance of the SER-SIT conditions can be found in Sobel [1981]. As a class of games, SER-SIT stochastic games were introduced by Parthasarathy et al. [1984]. They showed, among other results, that for this class of stochastic games: in the zero-sum case the limiting average value is independent of the initial state and both players have state independent stationary limiting average optimal strategies; in the general-sum case there exists a state independent stationary limiting average equilibrium. In this section we derive some results for SIT stochastic games, without using the SER-property. 


\subsubsection{THEOREM}

For any zero-sum SIT stochastic game the limiting average value $v^{1}$ is independent of the initial state: $v_{s}^{1}=v_{t}^{1}$ for all $s, t \in S$.

Furthermore both piayers have stationary limiting average optimal strategies.

\section{Proof:}

Let $\left\{x^{\beta}: \beta \in[0,1)\right\}$ be a sequence of stationary $\beta$-discounted optimal strategies for player 1 and let $x^{1}=\lim _{\beta \uparrow 1} x^{\beta}$.

Let $y^{*}$ be a stationary limiting average best reply for player 2 against $x^{\prime}$.

Let $S^{*}$ be the set of states $s$ with $\gamma^{1}\left(s, x^{1}, y^{*}\right)=v_{s}^{1}=\max _{t \in S} v_{t}^{1}$.

By the proof of theorem 2.4 .2 we have that $S^{*} \neq \varnothing$.

Now observe that $p\left(t \mid s, x_{s}^{1}, y_{s}\right)=0$ for all $s \in S^{*}, t \in S \backslash S^{*}$ and any $y \in Y^{*}$. This can be seen by the following argument. Suppose there were $s \in S^{*}, t \in S \backslash S^{*}$ and $y \in Y$ such that $p\left(t \mid s, x_{s}^{1}, y_{s}\right)>0$; let $g$ be the Markov strategy (definition 1.3.2) defined by using $y$ at stage 1 and $y^{*}$ at all stages $n \geqslant 2$; then it follows that $\gamma^{1}\left(s, x^{1}, g\right)<v_{s}^{1}$, which contradicts the optimality of $x^{1}$ in $s$.

Hence, if player 1 uses $x^{1}$ then the play will never leave the set of states $S^{*}$, once it is reached.

Take $s^{*} \in S^{*}$ and define $x^{*}$ by $x_{s}^{*}:=x_{s}^{1}$ for $s \in S^{*}$ and $x_{s}^{*}:=x_{s}^{1}$ for $s \in S \backslash S^{*}$. Now, for any initial state, if player 1 uses $x^{*}$, then after 1 stage the play will be in $S^{*}$ with probability 1 because we have state independent transitions. Since for $s \in S^{*}$ the strategies $x^{1}$ and $x^{*}$ are equal, the play will remain in $S^{*}$ forever. Hence we have that for any initial state $s$ and any $y \in Y$ :

$$
\gamma^{1}\left(s, x^{*}, y\right) \geqslant \gamma^{1}\left(s^{*}, x^{1}, y^{*}\right)=v_{s}^{1}=\max _{v \in S} v_{i}^{1} \text {. }
$$

This implies that $v_{s}^{1}=v_{t}^{1}$ for all $s, t \in S$ and $x^{*}$ is limiting average optimal. A limiting average optimal strategy for player 2 can be derived analogously.

\subsubsection{THEOREM}

For every general-sum SIT stochastic game there exists an almost stationary limiting average e-equilibrium.

\section{Proof:}

Let $\left\{\left(x^{\beta}, y^{\beta}\right): \beta \in[0,1)\right\}$ be a sequence of stationary $\beta$-discounted equilibria, converging to $\left(x^{1}, y^{1}\right)$.

By lemma 2.3.6 there exists $h^{*} \in\{1,2, \ldots, H\}$ (defined as in definition 2.2.1) such that $\gamma^{i h^{*}}\left(x^{1}, y^{l}\right) \geqslant \max V^{1 h} \geq V^{1 h^{*}}$ and $\gamma^{2 h^{*}}\left(x^{1}, y^{1}\right) \geq V^{2 h^{*}}$.

Take some $s^{*} \in S^{h^{*}}$. Then $p\left(t \mid s, x_{s}^{\|}, y_{s}^{1^{*}}\right)=0$ for $s \in S, t \in S \backslash S^{h^{*}}$, by the state independent transitions and by the irreducibility of $S^{h}$. Define $x^{*}$ by $x_{s}^{*}:=x_{s}^{1}$ for $s \in S^{h^{*}}$ and $x_{s}^{*}:=x_{s}^{1}$ for $s \in S \backslash S^{h^{*}}$. Similarly define $y^{*}$ by $y_{s}^{*}:=y_{s}^{1}$ for $s \in S^{h^{*}}$ and $y_{s}^{*}:=y_{s}^{1 .}$ for $s \in S \backslash S^{k^{*}}$. Then $\gamma^{k}\left(s, x^{*}, y^{*}\right) \geqslant V^{k h^{*}}$ for $k=1$ as well as for $k=2$ and for any initial state $s$, since for any initial state the play will be in $S^{h}$ after at most one stage.

Now using the fact that the limiting average value is independent of the initial 
state and using arguments similar to those in the proof of lemma 2.3 .4 , one can obtain an almost stationary limiting average $\epsilon$-equilibrium $(\varepsilon>0)$.

\subsection{REPEATED GAMES WITH ABSORBMVG STATES}

\subsubsection{DEFINITION}

In a stochastic game a state $s \in S$ is called an absorbing state if $p(s \mid s, i, j)=1$ for all $i \in\left\{1,2, \ldots, m_{s}\right\}$ and all $j \in\left\{1,2, \ldots, n_{s}\right\}$. A repeated game with absorbing states is a stochastic game for which all states except one are absorbing states.

In this monograph we have already seen several examples of repeated games with absorbing states: $1.6 .5,1.7 .4,1.7 .6,1.8 .6,2.3 .8,2.4 .5,2.4 .8,3.2 .3,3.2 .4$.

The class of zero-sum repeated games with absorbing states was first examined by Kohlberg [1974], who extended the work of Blackwell \& Ferguson [1968] on the big match (cf. example 1.7.4).

Kohlberg [1974] showed that the limiting average value exists for any zerosum repeated game with absorbing states. Using the techniques of Kohlberg [1974] and inspired by Sorin [1986], example 1.8.6, Vrieze \& Thuijsman [1989] showed the existence of e-equilibria for general-sum repeated games with absorbing states. In their proof Vrieze \& Thuijsman construct $\epsilon$-equilibrium strategies for which it may occur that one of the players has to adjust the mixed action he uses in the non-absorbing state at all stages. Since for a repeated game with absorbing states the condition of theorem 3.2.13 is necessarily fulfilled, the next theorem follows immediately.

\subsubsection{THEOREM}

For any general-sum repeated game with absorbing states there exists an almost stationary limiting average $\mathrm{e}$-equilibrium for each $\epsilon>0$.

Observe that this theorem provides $\mathrm{e}$-equilibrium strategies which are different from those in Vrieze \& Thuijsman [1989]. Observe also that for several other classes of stochastic games, e.g. unichain stochastic games, single-loop stochastic games (cf. Filar [1981-a]), the condition of theorem 3.2.13 is automatically fulfilled, which immediately provides the existence of almost stationary limiting average equilibria for such games. However ${ }_{*}$ as for the unichain stochastic games, several results can be derived more explicitly for repeated games with absorbing states.

In this section we make the assumption that all absorbing states are of size $1 \times 1$. With respect to the existence of limiting average $\epsilon$-equilibria this assumption can be made without loss of generality, since for each of the absorbing states the existence of a stationary limiting average equilibrium is obvious (cf. theorem 1.8.1). Hence each of the absorbing states can be contracted to a $1 \times 1$ absorbing state with payoff's according to some equilibrium.

We introduce the following notation. 


\subsubsection{NOTATION}

A repeated game with absorbing states can be given by one $m \times n$-matrix of which entry $(i, j)$ is

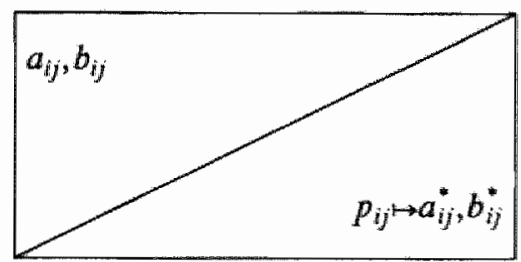

where $a_{i j}, b_{i j}, a_{i j}^{*}, b_{i j}^{*} \in \mathbb{R}$ and $p_{i j} \in[0,1]$.

The interpretation of the above notation is the following. If in the initial state, the non-absorbing one, player 1 chooses action $i$ and player 2 chooses action $j$, then player 1 (2) receives $a_{i j}\left(b_{i j}\right)$ and with probability $p_{i j}$ a transition takes place to an absorbing state where the players get $a_{i j}^{*}, b_{i j}$ from that stage on; with probability $1-p_{i j}$ the play remains in the initial state. Now a stationary strategy for player 1 is simply some $x \in \Delta^{m}$; for player 2 stationary strategies are elements $y \in \Delta^{n}$.

\subsubsection{LEMMA}

For a pair of stationary strategies $(x, y)$ we have:

$$
\begin{aligned}
& \text { a) } \gamma_{\beta}^{1}(x, y)=\frac{(1-\beta) \sum_{i} \sum_{j} x_{i} a_{i j} y_{j}+\beta \sum_{i} \sum_{j} x_{i} p_{i j} a_{i j}^{*} y_{j}}{1-\beta+\beta \sum_{i} \sum_{j} x_{i} p_{i j} y_{j}} \text { for } \beta \in[0,1) \text {. } \\
& \text { b) } \gamma^{1}(x, y)=\lim _{\beta \uparrow 1} \frac{(1-\beta) \sum \sum_{i} x_{i} a_{i j} y_{j}+\beta \sum_{i} \sum_{j} x_{i} p_{i j} a_{i j}^{*} y_{j}}{1-\beta+\beta \sum_{i} \sum_{j} x_{i} p_{i j} y_{j}} \text {. }
\end{aligned}
$$

c) Similar statements hold for player 2 's rewards.

\section{Proof:}

It is clear that (b) follows from (a) by lemma 1.5 .5 (d) and that (c) needs no further comment.

The formula in (a) follows directly from (cf. lemma 1.5 .3 (c)):

$$
\gamma_{\beta}^{1}(x, y)=(1-\beta) \sum_{i} \sum_{j} x_{i} a_{i j} y_{j}+\beta \sum_{i} \sum_{j} x_{i} p_{i j} a_{i j}^{*} y_{j}+\beta \sum_{i} \sum_{j} x_{i}\left(1-p_{i j}\right) y_{j} \gamma_{\beta}^{\prime}(x, y) \text {. }
$$

\subsubsection{DEFINITION}

A pair of stationary strategies $(x, y)$ is called absorbing if $\sum_{i} \sum_{j} x_{i} p_{i j} y_{j}>0$.

The next two lemmas can be derived directly from lemma 4.4.4. 


\subsubsection{LEMMA}

a) If $(x, y) \in \Delta^{m} \times \Delta^{n}$ is absorbing, then:

$$
\gamma^{\prime}(x, y)=\frac{\sum_{i} \sum_{j} x_{i} p_{i j} a_{i j}^{*} y_{j}}{\sum_{i} \sum_{j} x_{i} p_{i j} y_{j}} \text { and } \gamma^{2}(x, y)=\frac{\sum_{i} \sum_{j} x_{i} p_{i j} b_{i j}^{*} y_{j}}{\sum_{i} \sum_{j} x_{i} p_{i j} y_{j}} \text {. }
$$

b) If $(x, y) \in \Delta^{m} \times \Delta^{n}$ is non-absorbing, then:

$$
\gamma^{\prime}(x, y)=\sum_{i} \sum_{j} x_{i} a_{i j} y_{j} \text { and } \gamma^{2}(x, y)=\sum_{i} \sum_{j} x_{i} b_{i j} y_{j}
$$

\subsubsection{LEMMA}

Let $\left\{\left(x^{\beta}, y^{\beta}\right) \in \Delta^{m} \times \Delta^{n}: \beta \in[0,1)\right\}$ be a converging sequence with $\left(x^{\|}, y^{1}\right)=\lim \left(x^{\beta}, y^{\beta}\right)$ and with $\operatorname{Car}\left(x^{\beta}\right)$ and $\operatorname{Car}\left(y^{\beta}\right)$ independent of $\beta \in[0,1)$.

a) If $\left(x^{1}, y^{1}\right)$ is absorbing then $\left(x^{\beta}, y^{\beta}\right)$ is absorbing for $\beta \in[0,1)$ and $\gamma^{k}\left(x^{1}, y^{1}\right)=\lim _{\beta \uparrow 1} \gamma_{\beta}^{k}\left(x^{\beta}, y^{\beta}\right)$ for $k=1,2$.

b) If $\left(x^{\beta}, y^{\beta}\right)$ is non-absorbing for $\beta \in[0,1)$, then $\left(x^{1}, y^{1}\right)$ is non-absorbing and $\gamma^{k}\left(x^{1}, y^{\mathbb{1}}\right)=\lim _{\beta \uparrow 1} \gamma_{\beta}^{k}\left(x^{\beta}, y^{\beta}\right)$ for $k=1,2$.

\subsubsection{REMARK}

For the remainder of this section let $\left\{\left(x^{\beta}, y^{\beta}\right) \in \Delta^{m} \times \Delta^{n}: \beta \in[0,1)\right\}$ be a converging sequence of stationary $\beta$-discounted equilibria with $\left(x^{1}, y^{1}\right)=\lim _{\beta+1}\left(x^{\beta}, y^{\beta}\right)$ and with $\operatorname{Car}\left(x^{\beta}\right)$ and $\operatorname{Car}\left(y^{\beta}\right)$ independent of $\beta \in[0,1)$.

Let $V^{k}=\lim _{\beta \uparrow 1} \gamma^{k}\left(x^{\beta}, y^{\beta}\right)$ for $k=1,2$.

By lemma 1.6.4 we have the following fact.

\subsubsection{LEMMA}
a) If $x \in \Delta^{m t}$ and $\operatorname{Car}(x) \subset \operatorname{Car}\left(x^{\beta}\right), \beta \in[0,1)$, then $\gamma \beta\left(x, y^{\beta}\right)=\gamma_{\beta}^{l}\left(x^{1}, y^{\beta}\right)=\gamma_{\beta}^{1}\left(x^{\beta}, y^{\beta}\right)$.
b) If $y \in \Delta^{n}$ and $\operatorname{Car}(y) \subset \operatorname{Car}\left(y^{\beta}\right), \beta \in[0,1)$, then $\gamma_{\beta}^{2}\left(x^{\beta}, y\right)=\gamma_{\beta}^{2}\left(x^{\beta}, y^{1}\right)=\gamma_{\beta}^{2}\left(x^{\beta}, y^{\beta}\right)$.

\subsubsection{LEMMA}

a) If $x \in \Delta^{m}$ and either $\left(x, y^{1}\right)$ is absorbing or $\left(x, y^{\beta}\right)$ is non-absorbing, then $\gamma^{1}\left(x, y^{1}\right) \leqslant V^{1}$.

b) If $y \in \Delta^{n}$ and either $\left(x^{\prime}, y\right)$ is absorbing or $\left(x^{\beta}, y\right)$ is non-absorbing, when $\gamma^{2}\left(x^{1}, y\right) \leqslant V^{2}$.

\section{Proof:}

Using lemma 4.4 .7 and using that the pairs of strategies $\left(x^{\beta}, y^{\beta}\right)$ are $\beta$ discounted equilibria, we have (a) by:

$$
\gamma^{\prime}\left(x, y^{\prime}\right)=\lim _{\beta \uparrow 1} \gamma_{\beta}^{l}\left(x, y^{\beta}\right) \leqslant \lim _{\beta \uparrow 1} \gamma_{\beta}^{\prime}\left(x^{\beta}, y^{\beta}\right)=V^{1}
$$




\subsubsection{LEMMA}

If $\gamma^{k}\left(x^{1}, y^{1}\right) \geqslant V^{k}$ for $k=1,2$, then $\left(x^{1}, y^{1}\right)$ can be supplemented with retaliation threats to obtain an almast stationary $\mathrm{c}$-equilibrium.

\section{PROOF:}

Observe that by lemma 4.4 .10 neither player 1 nor player 2 can profit by an absorbing deviation from $\left(x^{1}, y^{1}\right)$. Hence retaliation threats are only needed to counter non-absorbing deviations. As in the proof of lemma 2.3.4, let $Y^{(n)}$ be the random variable (vector) denoting the action frequencies of player 2 in the initial state up to stage $n$ and let $y^{(n)}$ be a realization of $Y^{(n)}$. Let $X^{(n)}$ and $x^{(n)}$ be defined similarly for the action frequencies of player 1 .

Let $c>0$. Then, pretending absorption does not take place, for each $\alpha>0$ there is $\delta>0$ and $N_{\alpha \delta} \in \mathbb{N}$ such that:

$$
\begin{aligned}
& \text { Prob }_{x^{1}, y^{\prime}}\left\{\left\|X^{(n)}-x^{\|}\right\|>\alpha \text { for some } n \geqslant N_{\alpha \delta \delta}\right\}<\delta \text { and } \\
& \text { Prob }_{x^{\prime}, y^{\prime}}\left\{\left\|Y^{(n)}-y^{1}\right\|>\alpha \text { for some } n \geqslant N_{\alpha \delta}\right\}<\delta .
\end{aligned}
$$

Choose $\alpha \in(0, \epsilon / 8 M)$ and choose $\delta>0$ such that:

$$
(1-\delta)^{2}\left(\gamma^{k}\left(x^{1}, y^{1}\right)-2 \alpha M\right)-\left(1-(1-\delta)^{2}\right) M \geqslant \gamma^{k}\left(x^{1}, y^{1}\right)-\epsilon / 2 \text { for } k=1,2 \text {. }
$$

Define $\pi_{c}^{*}$ by:

a) use $x^{1}$ unless

i) player 2 chooses $j \notin \operatorname{Car}\left(y^{1}\right)$, or

ii) $\left\|y^{(n)}-y^{y}\right\|>\alpha$ for some $n \geqslant N_{\alpha \delta}$

b) if (i) or (ii) occurs, use some retaliation strategy $\pi_{c / 2}^{r}$ from that stage on.

Define $\sigma_{\varepsilon}^{*}$ analogouslly.

It can be verified that $\left(\pi_{c}^{*}, \sigma_{c}^{*}\right)$ is an almost stationary limiting average $\epsilon$ equilibrium.

The next lemma follows directly from lemma 4.4.7 and lemma 4.4.9.

\subsubsection{LEMMA}

If $\gamma^{2}\left(x^{1}, y^{1}\right)<v^{2}$, then $\left(x^{1}, y^{1}\right)$ is non-absorbing whereas $\left(x^{\beta}, y^{1}\right)$ is absorbing for all $\beta \in[0,1)$.

\subsubsection{DEFINITION}

If $\gamma^{2}\left(x_{\beta}^{1}, y^{1}\right) \leq \gamma^{2}$, then we define:

a) $\tilde{x}^{\beta}$ and $\tilde{x}^{*} \in \mathbb{R}^{m}$ by $(i \in\{1,2, \ldots, m\})$ :

$$
\begin{aligned}
& \tilde{x}_{i}^{\beta}:=\left\{\begin{array}{lll}
x_{i}^{\beta} & \text { if }\left(i, y^{1}\right) & \text { is non-absorbing } \\
0 & \text { if }\left(i, y^{1}\right) & \text { is absorbing }
\end{array}\right. \\
& \tilde{x}_{i}^{* \beta}:=\left\{\begin{array}{lll}
0 & \text { if }\left(i, y^{1}\right) & \text { is non -absorbing } \\
x_{i}^{\beta} & \text { if }\left(i, y^{1}\right) & \text { is absorbing }
\end{array}\right.
\end{aligned}
$$

b) $\underline{x}^{\beta}$ and $\underline{x}^{* \beta} \in \Delta^{m}$ by $(i \in\{1,2, \ldots, m\}): \underline{x}_{i}^{\beta}:=\frac{\tilde{x}_{i}^{\beta}}{\sum_{i} \tilde{x}_{i}^{\beta}}$ and $x_{i}^{* \beta}:=\frac{\tilde{x}_{i}^{* \beta}}{\sum_{i} \tilde{x}_{i}^{* \beta}}$. 
c) $x^{*}:=\lim _{\beta \uparrow 1} x^{* \beta}$ (which limit exists without loss of generality).

d) $\mu^{\beta}:=\frac{1-\beta}{1-\beta+\beta \sum_{i} \sum x_{j}^{\beta} p_{i j} y_{j}^{1}}$ and $\mu^{1}:=\lim _{\beta \uparrow 1} \mu^{\beta}$ (without loss of generality).

Observe that by these definitions we have that $x^{\beta}=\tilde{x}^{\beta}+\tilde{x}^{* \beta} ; \tilde{x}^{* \beta} \neq 0$ for all $\beta$; $\lim _{\beta \uparrow 1} \tilde{x}^{* \beta}=0 ; \lim _{\beta \uparrow 1} \tilde{x}^{\beta}=\lim _{\beta \uparrow 1} x^{\beta}=\lim _{\beta \uparrow 1} x^{\beta}=x^{1}$.

It is also clear that for all $\beta$ we have $\mu^{\beta} \in[0,1]$ and $\mu^{1} \in[0, \mathbb{1}]$.

\subsubsection{LEMMA}

If $\gamma^{2}\left(x^{1}, y^{1}\right)<V^{2}$ and $\mu^{1}$ and $x^{*}$ are as above, then $V^{2}=\mu^{1} \gamma^{2}\left(x^{1}, y^{1}\right)+\left(1-\mu^{1}\right) \gamma^{2}\left(x^{*}, y^{1}\right)$ and hence $\gamma^{2}\left(x^{*}, y^{\mathbb{1}}\right) \geqslant V^{2}$.

Proof:

From definition 4.4 .13 observe that $\sum_{i} \sum_{j} x_{j}^{\beta} p_{i j} y_{j}^{1}=\sum_{i} \sum_{j} \tilde{x}_{i}^{* \beta} p_{i j} y_{j}^{1}$ and $\sum_{i} \sum_{j} x_{i}^{\beta} p_{i j} b_{i j}^{*} y_{j}^{1}=\sum_{i} \sum_{j} \tilde{x}_{i}^{* \beta} p_{i j} b_{i j}^{*} y_{j}^{1}$. Using this, definition 4.4 .13 , lemma 4.4 .9 and lemma 4.4.4 we obtain:

$$
\begin{aligned}
V^{2} & =\lim _{\beta \uparrow 1} \gamma_{\beta}^{2}\left(x^{\beta}, y^{1}\right) \\
& =\mu^{1} \sum_{j} \sum_{j} x_{i}^{1} b_{i j} y_{j}^{1}+\lim _{\beta \uparrow 1} \frac{\beta \mu^{\beta}}{1-\beta} \sum_{i} \sum_{j} x_{i j}^{\beta} p_{i j} b_{i j}^{*} y_{j}^{1} \\
& =\mu^{1} \gamma^{2}\left(x^{1}, y^{1}\right)+\lim _{\beta \uparrow 1} \frac{\beta \mu^{\beta}}{1-\beta}\left(\sum_{i} \sum_{j} x_{i}^{\beta} p_{i j} y_{j}^{1}\right) \frac{\sum_{i} \sum_{j} \tilde{x}_{i}^{* \beta} p_{i j} b_{i j}^{*} y_{j}^{1}}{\sum_{i} \sum_{i} \tilde{x}_{i}^{* \beta} p_{i j} y_{j}^{1}} \\
& =\mu^{1} \gamma^{2}\left(x^{1}, y^{1}\right)+\lim _{\beta \uparrow 1}\left(1-\mu^{\beta}\right) \frac{\sum_{i} \sum_{j} x_{i}^{* \beta} p_{i j} b_{i j}^{*} y_{j}^{1}}{\sum_{i} \sum_{j} x_{i}^{* \beta} p_{i j} y_{j}^{1}} \\
& =\mu^{1} \gamma^{2}\left(x^{1}, y^{1}\right)+\left(1-\mu^{1}\right) \gamma^{2}\left(x^{*}, y^{1}\right) .
\end{aligned}
$$

Since $\gamma^{2}\left(x^{1}, y^{11}\right)<V^{2}$ and $\mu^{1} \in[0,1]$ we have that $\gamma^{2}\left(x^{*}, y^{1}\right) \geqslant V^{2}$.

\subsubsection{LEMMA}

If $\gamma^{2}\left(x^{1}, y^{1}\right)<V^{2}$ and $>0$, then $\left(x^{\lambda}, y^{1}\right)$, with $x^{\lambda}:=(1-\lambda) x^{1}+\lambda x^{*}$ and $\lambda \in(0,1)$, can be supplemented with retaliation threats to yield an almost stationary limiting average c-equilibrium for $\lambda$ sufficiently small.

PRoOF:

For each $\lambda \in(0,1)$ we have $\gamma^{2}\left(x^{\lambda}, y^{1}\right)=\gamma^{2}\left(x^{*}, y^{1}\right) \geq V^{2}$ and by lemmas 4.4.7 and 4.4.9: $\gamma^{1}\left(x^{\lambda}, y^{1}\right)=\gamma^{l}\left(x^{*}, y^{1}\right)=\lim _{\beta \uparrow 1} \gamma^{1}\left(x^{*}, y^{\beta}\right)=\lim _{\beta \uparrow 1} \gamma^{1}\left(x^{\beta}, y^{\beta}\right)=V^{1}$. 
Let again $Y^{(n)}$ be the random variable denoting the action frequencies of player 2 in the initial state up to stage $n$ and let $y^{(n)}$ be a realization of $Y^{(m)}$. Let $X^{(n)}$ be the random variable denoting the action frequencies of player 1 within $\operatorname{Car}\left(x^{1}\right)$ in the initial state up to stage $n$. Let $x^{(n)}$ be a realization of $X^{(m)}$. Let $\epsilon>0$. Then, pretending absorption does not take place, for each $\alpha>0$ and $\delta>0$ there is $N_{a b} \in \mathbb{N}$ such that:

$$
\begin{aligned}
& \text { Prob }_{x^{4}, y^{\prime}}\left\{\left\|X^{(n)}-x^{1}\right\|>\alpha \text { for some } n \geqslant N_{\alpha \delta}\right\}<\delta \text { and } \\
& {\text { Prob } x^{4}, y^{1}} \quad\left\{\left\|Y^{(n)}-y^{1}\right\|>\alpha \text { for some } n \geqslant N_{\alpha \delta}\right\}<\delta .
\end{aligned}
$$

Choose $\alpha \in(0, \epsilon / 8 M)$ and choose $\delta$ such that for $k=1,2$ :

$$
(1-\delta)^{4}\left(\gamma^{k}\left(x^{*}, y^{1}\right)-2 \alpha M\right)-\left(1-(1-\delta)^{4}\right) M \geqslant \gamma^{k}\left(x^{1}, y^{1}\right)-d / 2 .
$$

Choose $\lambda \in(0, \epsilon / 2 M)$ such that Prob $x_{x^{\lambda}, y^{\prime}}$ \{absorption before stage $\left.N_{a \delta}\right\}<\delta$. Choose $N_{\lambda} \in \mathbb{N}_{,} N_{\lambda}>N_{a \delta}$, such that

Prob $_{x^{\lambda}, y^{3}}\left\{\right.$ absorption before stage $\left.N_{\lambda}\right\} \geqslant 1-\delta$.

Define $\pi_{i}^{*}$ by:

a) use $x^{\lambda}$ unless

i) player 2 chooses $j \notin \operatorname{Car}\left(y^{1}\right)$, or

ii) $\left\|y^{(n)}-y^{\mathbb{1}}\right\|>\alpha$ for some $n \geqslant N_{\alpha \delta}$

iii) at stage $N_{\lambda}$ play is still in the initial state

b) if (i), (ii) or (iii) occurs, use some retaliation strategy $\pi_{k / 2}^{r}$ (cf. 1.8.5).

Define $\sigma_{\varepsilon}^{*}$ in a similar way.

Now $\left(\pi_{\epsilon}^{*}, \sigma_{\epsilon}^{*}\right)$ is an almost stationary limiting average $\epsilon$-equilibrium.

Observe that example 3.2 .4 is an illustration of the lemmas 4.4.14 and 4.4.15; in that example $\mu^{1}=3 / 3$. The next example illustrates that one may also have $\gamma^{2}\left(x^{1}, y^{1}\right)<V^{2}$ and $\mu^{1}=0$.

\subsubsection{EXAMPLE}

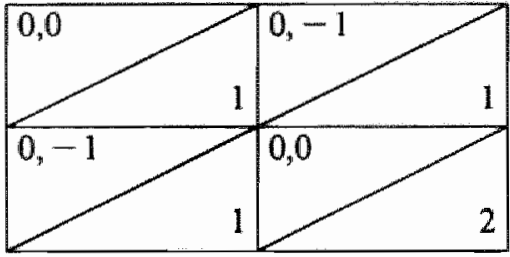

State 1

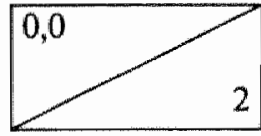

State 2

For this example stationary $\beta$-discounted equilibria are for instance given by $\left(x^{\beta}, y^{\beta}\right)=\left(\left(\frac{1-\sqrt{1-\beta}}{\beta}, \frac{-1+\beta+\sqrt{1-\beta}}{\beta}\right),(0,1)\right)$, with $\beta \in[0,1)$.

Now $\gamma^{2}\left(x^{1}, y^{1}\right)=-1$, whereas $V^{2}=\lim _{\beta \uparrow 1} \gamma_{\beta}^{2}\left(x^{\beta}, y^{\beta}\right)=\lim _{\beta \uparrow 1} \frac{1-\beta-\sqrt{1-\beta}}{\beta}=0$. Hence $x^{*}=(0,1)$ and $\gamma^{2}\left(x^{*}, y^{11}\right)=0 ; \mu^{11}=0$.

Examples that illustrate lemma 4.4.11 are for instance 2.3.8 and 3.2.3. 


$$
\text { . }
$$




\section{Chapter 5}

\section{The total reward criterion in zero-sum stochastic games}

\subsection{INTRODUCTION}

In chapter 1 we briefly dealt with the total reward criterion. This criterion has been introduced in Thuijsman \& Vrieze [1987] and Vrieze \& Thuijsman [1987]. Based on these papers, we examine this criterion as well as its relations with the $\beta$-discounted reward criterion and with the limiting average reward criterion for the zero-sum case.

In section 5.2 we give several examples to support our choice for defining total rewards by $\liminf _{N \rightarrow \infty} \frac{1}{N} \sum_{m=1}^{N} \sum_{n=1}^{m} E_{s m o}\left[R^{1}(n)\right]$, as is done in definition 1.4.4. Furthermore these examples lead to the conclusion that a certain property should be fulfilled if we want that the total value, whenever it exists, is finite. This property is that the limiting average value should be 0 (for all initial states) and both players should have stationary limiting average optimal strategies.

For the existence of stationary limiting average optimal strategies, several characterizations have been given (cf. Sobel [1971], Bewley \& Kohlberg [1978], Filar \& Schultz [1986], Schultz [1987], Vrieze [1987-a]). Several of these characterizations are by means of mathematical programs (see chapter 6). By means of equations similar to the Shapley-equation (cf. 1.7.3), the existence of stationary limiting average optimal strategies has been characterized by Vrieze [1987a].

In section 5.3 we extend Vrieze's result by characterizing the existence of stationary total optimal strategies for stochastic games with the above property.

In section 5.4 we give an example to illustrate that, even with the above property, history dependent strategies are indispensable to achieve total :optimality. This indicates that the total reward criterion and the limiting average criterion have similar features.

In section 5.5 we show that examining total rewards in a stochastic game is equivalent to examining limiting average rewards in a related stochastic game with countable state space. 


\subsection{THE TOTAL REWARD CRITERION}

For the $\beta$-discounted reward criterion the emphasis is on near-future payoffs, while for the limiting average reward criterion the emphasis is on far-future payoffs. In certain situations however, it would be more appropriate to use an intermediate criterion, where both near-future and far-future payoffs are equally important. There are several ways of defining such an intermediate criterion. Recently Krass et al. [1987] and Filar \& Vrieze [1989] examined a criterion which is a "weighted combination' of the $\beta$-discounted reward criterion and the limiting average reward criterion. In this chapter however we look at the total reward criterion, which is also an intermediate criterion. To see why this total reward criterion is interesting, think for instance of stopping stochastic games, as introduced in Shapley [1953], where for all $s, i$ and $j$ there is a small probability of stopping or equivalently, a small probability of moving to an absorbing state where the payoffs are 0 . In such games the limiting average reward is necessarily equal to $D$ for any pair of strategies and hence the limiting average criterion does not seem to be a suitable criterion to examine the game. Instead of discounting with some factor $\beta \in[0,1)$ one would simply like to take the sum of all payoffs for any play in such a game. The same holds for the next example although it is no stopping stochastic game.

\subsubsection{EXAMPLE}

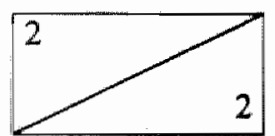

State 1

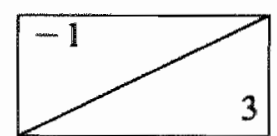

State 2

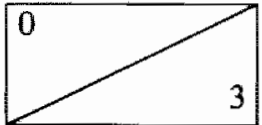

State 3

The payoffs in this example, like those in the other examples in this section, are the payoffs to player 1 to be paid by player 2 .

For this example it is clear that the limiting average reward (for the unique strategies) is 0 for all initial states. However, it seems reasonable that player 1 would prefer to start in state 1 , whereas player 2 would prefer initial state 2 .

In the following example we can also imagine that player 1 would prefer to start in state 1 while player 2 would prefer to start in state 2 . The limiting average reward is again 0 for both initial states.

\subsubsection{EXAMPLE}

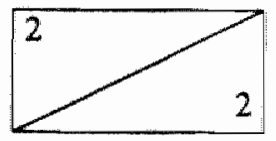

State 1

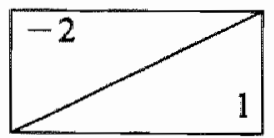

State 2 
For the play starting in state 1 the sequence of partial sums of payoffs would be $(2,0,2,0,2, \ldots)$. Thus, on the average, player 1 owns 1 , whereas it is clear that the limiting average reward is 0 for initial state 1 . Similarly, for the play starting in state 2 , player 1 would own -1 on the average. The $\beta$-discounted reward for initial state 1 is $2(1-\beta) \sum_{n=1}^{\infty}(-\beta)^{n-1}=2(1-\beta) /(1+\beta)$. Leaving out normalization by $1-\beta$ gives $2 /(1+\beta)$, which converges to 1 as $\beta$ goes to 1 .

These examples and the fact that, if $\sum_{n=1}^{\infty} E_{s \text { ซ }}\left[R^{1}(n)\right]$ exists in $\mathbb{R} \cup(-\infty,+\infty)$ then it is equal to $\liminf _{N \rightarrow \infty} \frac{1}{N} \sum_{m=1}^{N} \sum_{n=1}^{n=1} E_{s r r}\left[R^{1}(n)\right]$, lead us to define total rewards by the latter expression (cf. definition 1.4.4). Here we use "lim inf" since for non-stationary strategies 'lim" may fail to exist in $\mathbb{R} \cup\{-\infty,+\infty\}$. We chose "lim inf" since, like for the limiting average criterion, this reflects a kind of 'worst case view' of player 1. However we could also have chosen 'lim sup' or any convex combination of 'lim inf' and 'lim sup'.

In lemma 1.5.7 we have shown that for any pair of stationary strategies $\lim _{N \rightarrow \infty} \frac{1}{N} \sum_{m=1}^{N} \sum_{n=1}^{m} E_{s x y}\left[R^{1}(n)\right]$ is finite on condition that $\gamma^{1}(x, y)=0$. It makes no sense to define a total reward evaluation by $E_{s \text { s }}$ [ $\lim _{N \rightarrow \infty} \inf \frac{1}{N} \sum_{m=1}^{N} \sum_{n=1}^{m} R^{\prime}(n)$ ], since the following example, which was communicated to us by Neyman [1986], shows that this alternative definition does not express what we would like to call a total reward.

\subsubsection{EXAMPLE}

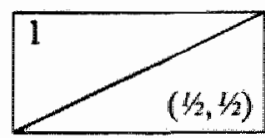

State 1

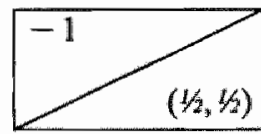

State 2

For this example we have that $E\left[\liminf _{N \rightarrow \infty} \frac{1}{N} \sum_{m=1}^{N} \sum_{n=1}^{m} R^{1}(n)\right]=-\infty$ for both initial states, since with probability 1 , for any realization of the random walk, $\liminf _{N \rightarrow \infty} \frac{1}{N} \sum_{m=1}^{N} \sum_{n=1}^{m} r^{1}(n)=-\infty$

We now turn to some properties of the total reward criterion.

\subsubsection{THEOREM}

For any pair of strategies $(\pi, 0) \in \Pi \times \Sigma$ we have:

$$
\lim _{\beta \uparrow 1} \inf _{i}(1-\beta)^{-1} \gamma_{\beta}^{1}(\pi, \sigma) \geqslant \gamma_{T}^{1}(\pi, \sigma) .
$$


PROOF:

Let $\left\{a_{n} \in \mathbb{R}: n \in \mathbb{N}\right\}$. Then:

$$
\liminf _{\beta \uparrow 1}(1-\beta) \sum_{m=1}^{\infty} \beta^{n-1} a_{n} \geqslant \liminf _{N \rightarrow \infty} \frac{1}{N} \sum_{m=1}^{N} a_{n}
$$

as can be found in Derman [1970], based on Widder [1946].

For each $\beta \in[0,1)$ it holds that:

$$
(1-\beta)^{-1} \sum_{m=1}^{\infty} \beta^{m-1} a_{m}=\sum_{m=1}^{\infty} \beta^{m-1} \sum_{n=1}^{m} a_{m}
$$

(this is easy to verify, cf. page 37 in Kallenberg [1983]).

Hence we conclude that for all $s \in S$ and all $\pi, \sigma \in \Pi \times \Sigma$ :

$$
\begin{aligned}
\underset{\beta \uparrow 1}{\liminf (1-\beta)^{-1}} \gamma_{\beta}^{1}(s, \pi, \sigma) & =\liminf _{\beta \uparrow 1} \sum_{n=1}^{\infty} \beta^{n-1} E_{s \pi \sigma}\left[R^{1}(n)\right] \\
& =\liminf _{\beta \uparrow 1}(1-\beta) \sum_{m=1}^{\infty} \beta^{m-1} \sum_{n=1}^{m} E_{s \pi \sigma}\left[R^{1}(n)\right] \\
& \geq \liminf _{N \rightarrow \infty} \frac{1}{N} \sum_{m=1}^{N} \sum_{n=1}^{m} E_{s \pi \sigma}\left[R^{1}(n)\right]=\gamma_{T}^{1}(s, \pi, \sigma) .
\end{aligned}
$$

\subsubsection{THEOREM}

For a zero-sum stochastic game with limiting average value a for all initial states) let $y^{*} \in Y$ be a stationary limiting average optimal strategy.

Then there exists a pure stationary total best reply against $y^{*}$ for player 1 and $\gamma_{T}^{\|}\left(s, \pi, y^{*}\right)<\infty$ for all $\pi \in \Pi$ and all $s \in S$.

\section{Proof:}

Since there are only finitely many pure stationary strategies, we can assume (by taking some subsequence) that there is a pure stationary strategy $x^{*}$ such that $x^{*}$ is a $\beta$-discounted best reply against $y^{*}$ for all $\beta$ close to 1 .

By lemma 1.5 .5 and by theorem 1.7.7 we have $0 \geqslant \gamma^{\prime}\left(x^{*}, y^{*}\right)=\lim _{\beta \uparrow 1} \gamma_{\beta}^{\prime \prime}\left(x^{*}, y^{*}\right) \geqslant$ $\lim _{\beta \uparrow 1} v_{\beta}^{*}=v^{1}=0$, and hence $\gamma^{1}\left(x^{*}, y^{*}\right)=0$.

Now let $\pi \in \Pi$. Then by theorem 5.2 .4 and lemma 1.5 .7 , we have for all $s \in S$ :

$$
\begin{aligned}
\gamma_{T}^{1}\left(s, \pi, y^{*}\right) & \leqslant \liminf _{\beta \uparrow 1}(1-\beta)^{-1} \gamma_{\beta}^{1}\left(s, \pi, y^{*}\right) \\
& \leqslant \liminf _{\beta \uparrow 1}(1-\beta)^{-1} \gamma_{\beta}^{1}\left(s, x^{*}, y^{*}\right) \\
& =\lim _{\beta \uparrow 1}(1-\beta)^{-1} \gamma_{\beta}^{1}\left(s, x^{*}, y^{*}\right) \\
& =\gamma_{T}^{1}\left(s, x^{*}, y^{*}\right) \\
& =\left(I-P\left(x^{*}, y^{*}\right)+Q\left(x^{*}, y^{*}\right)\right)_{s}^{-1} r^{k}\left(x^{*}, y^{*}\right)<\infty
\end{aligned}
$$


Closely related to theorem 5.2 .5 is the next theorem.

\subsubsection{THEOREM}

If for a zero-sum stochastic game with limiting average value 0 there are strategies $x^{*} \in X$ and $y^{*} \in Y$ which are uniform $\beta$-discounted optimal fi.e. $\beta$ discounted optimal for all $\beta$ close to 1), then the total value exists, $x^{*}$ and $y^{*}$ are total optimal and $v_{T}^{1}=\lim _{\beta \uparrow 1}(1-\beta)^{-1} v_{\beta}^{1}$.

\section{Proof:}

From lemmas $1.5 .5,1.6 .2$ and from theorem 1.7 .7 it follows that stationary uniform $\beta$-discounted optimal strategies are also limiting average optimal. Previously this result has been shown by Bewley \& Kohlberg [1978]. Hence $x$ * and $y^{*}$ are limiting average optimal and $\gamma^{1}\left(x^{*}, y^{*}\right)=0$. Now the result follows from the proof of theorem 5.2.5, since $x^{*}$ is and $y^{*}$ are $\beta$-discounted best replies against each other for all $\beta$ close to 1 .

\subsubsection{THEOREM}

If for a zero-sum stochastic gume both players have stationary total optimal strategies and $v_{T}^{1}$ is finite, then $v_{T}^{9}=\lim _{\beta \uparrow l}(1-\beta)^{-1} v_{\beta}^{1}$.

\section{Proof:}

Let $x^{*}$ be a stationary total optimal strategy for player 1 . Since there are only finitely many pure stationary strategies, there is, using lemma 1.6.2, a pure stationary strategy $y^{p}$ which is a $\beta$-discounted best reply for player 2 against $x^{*}$ for all $\beta$ close to 1 .

Now let $\epsilon>0$. Then for $\beta$ close to 1 we have, using theorem 5.2.4: $(1-\beta)^{-1} v_{\beta}^{1} \geqslant(1-\beta)^{-1} \gamma_{\beta}^{1}\left(x^{*}, y^{p}\right) \geqslant \gamma_{T}^{1}\left(x^{*}, y^{p}\right)-\epsilon 1_{z} \geqslant \nu_{T}^{1}-\epsilon 1_{z}$. Similarly one can show that $(1-\beta)^{-1} v_{\beta}^{1} \leqslant \gamma_{T}^{1}+\epsilon 1_{z}$ for $\beta$ close to 1 .

Observe that the above theorems imply that, under the condions in the theorems, $v_{T}^{1}$ equals $\alpha_{\mathcal{N}}$, the coefficient for $(1-\beta)$ in the power series expansion of $v_{\beta}^{1}$ in fractional powers of $(1-\beta)$. So both the limiting average value and the total value appear in this expansion (cfi. theorem 1.7.5).

It is evident that, if for a zero-sum stochastic game the limiting average value is not equal to 0 for some initial state $s$, then the total value $v_{T}(s)$ will either be $+\infty$ or $-\infty$. However, the existence of the total value in $\mathbb{R} \cup\{-\infty,+\infty\}$ for some initial state is not guaranteed by the limiting average value equalling 0 for the same initial state. Take for instance the next example. 


\subsubsection{EXAMPLE}

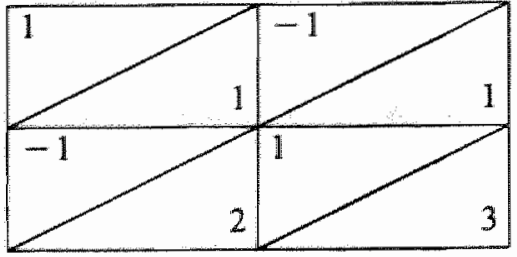

State 1

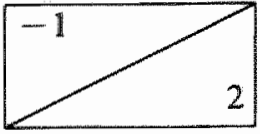

State 2

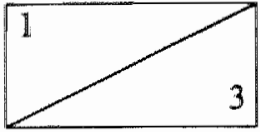

State 3

This example is essentially the big match of Blackwell \& Ferguson [1968], example 1.7.4. The limiting average value for initial state 1 is 0 in this example. For this stochastic game player 1 has no limiting average optimal strategy (cf. 1.7.4. (c)). Hence for each strategy $\pi \in \Pi$ there is some $\sigma \in \Sigma$ such that $\liminf _{N \rightarrow \infty} \frac{1}{N} \sum_{n=1}^{N} E_{1 \pi \sigma}\left[R^{1}(n)\right]<0$ and thus for those strategies we find $\left.\liminf _{N \rightarrow \infty} \frac{1}{N} \sum_{m=1}^{N} \sum_{n=1}^{n=1} E_{1 m 0} \llbracket R^{\prime}(n)\right]=-\infty$. It is easy to verify that with $y^{*}=(1 / 2,1 / 2)$ we have that $\gamma_{T}^{\prime}\left(1, \pi, y^{*}\right)=0$ for all $\pi \in \Pi$. Consequently, the total value does not exist for initial state 1 since we have $\sup _{\pi} \inf _{\sigma} \gamma_{T}^{1}(1, \pi, \sigma)=-\infty<0=\inf _{\sigma} \sup _{\pi} \gamma_{T}^{1}(1, \pi, \sigma)$.

The next example illustrates a curious phenomenon: even if the limiting average value equals 0 for all initial states and both players have limiting average optimal strategies, then the total value may still be infinite.

\subsubsection{EXAMPLE}

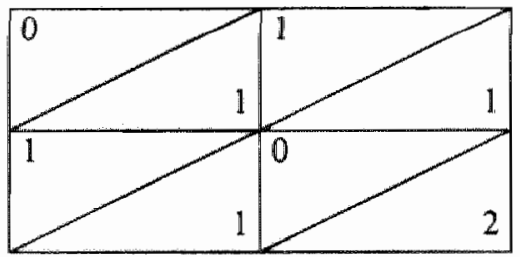

State 1

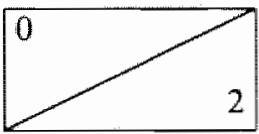

State 2

For this stochastic game the limiting average value is 0 for both initial states. For player 1 all strategies are limiting average optimal; for player 2 the stationary strategy $(1-\epsilon, \epsilon)$, with $\epsilon \in(0,1)$, is limiting average $\epsilon$-optimal. However here, as for any other stochastic game with a state independent value, player 2 possesses a Markov strategy (cf. definition 1.3.2) that is limiting average optimal. Such a limiting average optimal Markov strategy can for instance be obtained by using:

a one-stage optimal strategy at the first stage, followed by 
a two-stage optimal strategy at the next two stages, followed by

a three-stage optimal strategy at the next three stages, etc.

That such a strategy is optimal can be shown by using that $\lim _{N \rightarrow \infty} \frac{v_{s}^{(N)}}{N}=v_{s}^{1}$ is independent of $s \in S$, where $v_{s}^{(N)}$ denotes the value of the $N$-stage game starting in $s$ (cf. Mertens \& Neyman [1981], Bewley \& Kohlberg [1976, 1978] and Vrieze [1987-a]).

To show that the total value for initial state 1 is $+\infty$, observe that by using the stationary strategy $(1-\epsilon, \epsilon)$, with $\epsilon \in(0,1)$, player l's total reward will be at least $1 /(1-\epsilon)$ against any strategy of player 2 . Hence, letting $\epsilon$ tend to 0 we get the result that, although player 2 can keep the limiting average reward at 0 , the total reward value is $+\infty$.

It can even be verified for this example that player 1 can guarantee a total reward $+\infty$ by using the Markov strategy $f^{*}$ defined by: if at stage $n$ the play is stilli in state 1 , then use the mixed action $(n /(n+1), 1 /(n+1))$, for all $n \in \mathbb{N}$. Now $\gamma_{T}^{1}\left(1, f^{*}, \sigma\right)=+\infty$ for any strategy $\sigma \in \mathrm{\Sigma}$.

The above examples illustrate that only with the following property $(P)$ we can expect the total value, if it exists, to be finite.

\subsubsection{DEFINITION}

We say that the stochastic game has property $P$ if the limiting average value is 0 (for all initial states) and if, moreover, both players have stationary limiting average optimal strategies.

For a zero-sum stochastic game with property $P$, the total reward criterion can be seen as a refinement of the limiting average reward criterion, since it is obvious that for such a game a total (c-)optimal strategy is necessarily limiting average optimal.

For the remainder of this chapter we focus on zero-sum stochastic games with property $P$.

\subsection{STOCHASTIC GAMES AND OPTIMAL STATIONARY STRATEGIES}

It is well-known that in any zero-sum stochastic game there exist stationary $\beta$ discounted optimal strategies (cf. theorem 1.7.3). Furthermore it is well-known that stationary limiting average optimal strategies do not always exist (cf. example 1.7.4). Vrieze [1987-a] gives the following characterization for the existence of stationary limiting average optimal strategies.

\subsubsection{THEOREM}

For any zero-stum stochastic game both players have stationary limiting average optimal strategies if and only if there exist $\alpha, \delta_{1}, \delta_{2} \in \mathbb{R}^{z}$ such that for all $s \in S$ :

a) $\alpha_{s}=\underset{A_{s} \times B_{s}}{\operatorname{val}}\left[\sum_{i=1}^{z} p(t \mid s, i, j) \alpha_{t}\right]$ 
b) $\alpha_{s}+\delta_{1 s}=\operatorname{val}_{o_{u}^{*} \times B_{s}}\left[r^{1}\left(s, i_{s} j\right)+\sum_{t=1}^{z} p(t \mid s, i, j) \delta_{1 u}\right]$

c) $\alpha_{s}+\delta_{2 s}=\underset{A_{4} \times o_{2}}{\operatorname{val}}\left[r^{1}\left(s, i_{j} j\right)+\sum_{t=1}^{z} p(t \mid s, i, j) \delta_{2 t}\right]$.

Here $A_{s}=\left\{1,2, \ldots, m_{s}\right\}, B_{s}=\left\{1,2, \ldots, n_{s}\right\}$ and $O_{1 s}$ (resp. $\left.O_{2 s}\right)$ is the set of optimal mixed actions for player 1 (resp. 2) in the matrix game $\left[\sum_{t=1}^{z} p(t \mid s, i, j) \alpha_{t}\right]_{i=1}^{m_{t}}{ }_{1}^{n_{t}}=1$.

Shapley \& Snow [1950] showed that for each player the set of optimal mixed actions of a matrix game is a bounded polyhedron, with a finite number of extreme points. In view of this result $\underset{O_{i} \times B_{s}}{v a l}\left[r^{1}(s, i, j)+\sum_{i=1}^{*} p(t \mid s, i, j) \delta_{1 t}\right]$ is the value of a polyhedral game (cf. Wolfe [1956]), where for $(x, y) \in O_{1 s} \times B_{s}$ the payoff equals $\sum_{i=1}^{m_{s}} \sum_{j=1}^{n_{s}} x_{i}\left(r^{1}(s, i, j)+\sum_{i=1}^{z} p(t \mid s, i, j) \delta_{\| r}\right) y_{j}$.

The expression $\underset{A, \times O_{2}}{\text { val }}\left[r^{1}(s, i, j)+\sum_{i=1}^{z} p(t \mid s, i, j) \delta_{2 t}\right]$ should be interpreted in a similar way.

For every solution $\left(\alpha, \delta_{1}, \delta_{2}\right)$ of the equations in theorem 5.3.1 it can be verified that $\alpha=v^{1}$, the limiting average value of the stochastic game. Furthermore, given such a solution $\left(\alpha, \delta_{1}, \delta_{2}\right)$, a stationary limiting average optimal strategy $x^{*}$ for player 1 can be constructed by letting $x_{s}^{*}$ be an optimal mixed action for player 1 in the polyhedral game $\left[r^{1}(s, i, j)+\sum_{i=1}^{z} p(t \mid s, i, j) \delta_{1 i}\right] o_{u s} \times B_{s}$, for each $s \in S$. A stationary limiting average optimal strategy $y^{*}$ for player 2 can be found in a similar way. An example in Vrieze [1987-a] shows that there may be stationary limiting average optimal strategies which cannot be found from any solution $\left(\alpha, \delta_{1}, \delta_{2}\right)$.

Observe that theorem 5.3.1 implies that a zero-sum stochastic game has property $P$ (cf. definition 5.2.10) if and only if there exists $\delta \in \mathbb{R}^{z}$ such that:

$$
\delta_{s}=\underset{A_{u} \times B_{s}}{v a l}\left[r^{1}(s, i, j)+\sum_{i=1}^{\sum} p(t \mid s, i, j) \delta_{t}\right] \text {, for each } s \in S .
$$

We now give an analogue of theorem 5.3.1 for the existence of stationary total optimal strategies for stochastic games with property $P$.

\subsubsection{THEOREM}

For any zero-sum stochastic gume with property $P$ the total value exists in $\mathbb{R}^{z}$ and both players have stationary total optimal strategies if and only if there exist $\alpha, \delta_{1}, \delta_{2} \in \mathbb{R}^{2}$ and $\lambda \geq 0$ such that for all $s \in S$ :
a) $\alpha_{s}=\operatorname{val}_{A_{s} \times B_{s}}\left[r^{1}(s, i, j)+\sum_{i=1}^{z} p(r \mid s, i, j) \alpha_{t}\right]$
b) $\alpha_{s}+\delta_{1 s}=\underset{o_{1} \times B_{s}}{y a l}\left[\lambda r^{1}(s, i, j)+\sum_{t=1}^{z} p(t \mid s, i, j) \delta_{1 t}\right]$
c) $\alpha_{s}+\delta_{2 s}=\underset{A_{1} \times O_{2}^{*}}{v a l}\left[\lambda r^{-1}(s, i, j)+\sum_{t=1}^{j} p(t \mid s, i, j) \delta_{2 t}\right]$. 
Here $O_{1 s}^{*}$ and $O_{2 s}^{*}$ are the sets of optimal mixed actions for the respective players for the matrix game in (a), and the games in (b) and (c) are again polyhedral games.

\section{Proof:}

THE "IF"-PART:

Suppose there are $\alpha, \delta_{1}, \delta_{2} \in \mathbb{R}^{z}$ and $\lambda \geqslant 0$ such that for each $s \in S$ the statements (a), (b) and (c) hold. Let $x_{s}^{*}$ be an optimal mixed action for player 1 in the polyhedral game $\left[\lambda r^{1}\left(s, i_{j} j\right)+\sum_{i=1}^{z} p(t \mid s, i, j) \delta_{t}^{1}\right]_{o_{i s}^{i} \times B_{j}}$, for each $s \in S$ and let $y \in Y$.

From (a) we conclude that $\alpha \leqslant r^{1}\left(x^{*}, y\right)+P\left(x^{*}, y\right) \alpha$ and hence it follows that $0 \leqslant Q\left(x^{*}, y\right) r^{1}\left(x^{*}, y\right)=\gamma^{1}\left(x^{*}, y\right)$, so $x^{*}$ is limiting average optimal.

It is clear that if $\gamma^{1}\left(s, x^{*}, y\right)>0$ then $\gamma_{T}^{\frac{1}{1}}\left(s, x^{*}, y\right)=\infty>\alpha_{s}$.

So let $A \subset S$, be the set of states with $\gamma^{1}\left(s, x^{*}, y\right)=0$.

Then $p\left(t \mid s, x^{*}, y\right)=0$ for all $t \in A^{c}$ and $s \in A$. Let $\alpha^{A}, \delta_{1}^{A}, r^{1}\left(x^{*}, y\right)^{A}, P\left(x^{*}, y\right)^{A}$, $Q\left(x^{*}, y\right)^{A}$ be restrictions to states in $A$.

We have $0=\gamma^{1}\left(x^{*}, y\right)^{A}=Q\left(x^{*}, y\right)^{A} r^{A}\left(x^{*}, y\right)^{A}$.

From (b) we conclude that $\alpha^{A}+\delta_{1}^{A} \leqslant \lambda r^{1}\left(x^{*}, y\right)^{A}+P\left(x^{*}, y\right)^{A} \delta_{1}^{A}$ and hence it follows that $Q\left(x^{*}, y\right)^{A} \alpha^{A} \leqslant \lambda Q\left(x^{*}, y\right)^{A} r^{1}\left(x^{*}, y\right)^{A}=0$.

From (a) we have $\alpha^{A} \leqslant r^{1}\left(x^{*}, y\right)^{A}+P\left(x^{*}, y\right)^{A} \alpha^{A}$ which implies that:

$\alpha^{A} \leqslant\left(\sum_{n=1}^{m}\left(P\left(x^{*}, y\right)^{A}\right)^{n-1} r^{1}\left(x^{*}, y\right)^{A}\right)+\left(P\left(x^{*}, y\right)^{A}\right)^{m} \alpha^{A}$ for all $m \in \mathbb{N}$.

This implies:

$\alpha^{A} \leqslant\left(\frac{1}{N} \sum_{m=1}^{N} \sum_{n=1}^{m}\left(P\left(x^{*}, y\right)^{A}\right)^{n-1} r^{1}\left(x^{*}, y\right)^{A}\right)+\frac{1}{N} \sum_{m=1}^{N}\left(P\left(x^{*}, y\right)^{A}\right)^{m} \alpha^{A}$

for all $N \in \mathbb{N}$. Letting $N$ tend to infinity and using that $Q\left(x^{*}, y\right)^{A} \alpha^{A} \leqslant 0$ we get: $\alpha^{A} \leqslant \gamma_{T}^{\frac{1}{T}}\left(x^{*}, y\right)^{A}$.

By theorem 5.2 .5 we derive $\inf _{a} \gamma_{T}^{1}\left(x^{*}, \sigma\right) \geqslant \inf _{y} \gamma_{T}^{l}\left(x^{*}, y\right) \geqslant \alpha$ and hence $\sup _{\pi} \inf _{\sigma} \gamma \frac{1}{T}(\pi, \sigma) \geqslant \alpha$

Similarly we can show inf $\sup _{\pi} \gamma(\pi, \sigma) \leqslant \alpha$, and thus $\nu_{T}^{1}=\alpha \in \mathbb{R}^{2}$ and both players have stationary total optimal strategies.

THE 'ONLY IF'-PART:

Suppose that the total value $v_{\eta}^{1}$ exists in $\mathbb{R}^{2}$ and that both players have stationary total optimal strategies $x^{*} \in X$ and $y^{*} \in Y$.

Then $v_{T}^{1}=r^{1}\left(x^{*}, y^{*}\right)+P\left(x^{*}, y^{*}\right) v_{T}^{1}$ in view of lemma 1.5 .7 (b), since $Q\left(x^{*}, y^{*}\right) r^{1}\left(x^{*}, y^{*}\right)=0$. By the optimality of $x^{*}$ and $y^{*}$ :

$v_{T}^{1} \leqslant r^{1}\left(x^{*}, y\right)+P\left(x^{*}, y\right) v_{T}^{1}$ for all $y \in Y$, as well as

$v_{T}^{1} \geqslant r^{1}\left(x, y^{*}\right)+P\left(x, y^{*}\right) v_{T}^{1}$ for all $x \in X$.

Hence $v_{T}^{1}(s)=\underset{A_{s} \times B_{s}}{v a l}\left[r^{1}(s, i, j)+\sum_{s=1}^{z} p(t \mid s, i, j) v_{T}^{1}(t)\right]$ and $x_{s}^{*}$ and $y_{s}^{*}$ are optimal mixed actions in this matrix game for each $s \in S$. 
Now let $y \in Y$.

Since $\gamma_{T}^{1}\left(x^{*}, y\right) \geqslant \nu_{T}^{1}>-\infty$ it follows that $\gamma^{1}\left(x^{*}, y\right)=Q\left(x^{*}, y\right) r^{1}\left(x^{*}, y\right) \geqslant 0$. Let again $A$ be the set of states $s$ with $\gamma^{1}\left(s, x^{*}, y\right)=0$, and let $B=S \backslash A$. Then $p\left(t \mid s, x_{s}^{*}, y_{s}\right)=0$ for all $s \in A, t \in B$. Using similar notations as above we have (using lemma 1.5.2 and using $Q\left(x_{N}^{*}, y\right)^{A} r^{P}\left(x^{*}, y\right)^{A}=0$ ):

$Q\left(x^{*}, y\right)^{4} \gamma_{T}^{1}\left(x^{*}, y\right)^{A}=\lim _{N \rightarrow \infty} \frac{1}{N} \sum_{m=1}^{N} \sum_{n=1}^{m} Q\left(x^{*}, y\right)^{A} r^{1}\left(x^{*}, y\right)^{A}=0$.

Because $v_{T}^{A} \leqslant \gamma_{T}^{1}\left(x^{*}, y\right)^{A}$ we derive $Q\left(x^{*}, y\right)^{A} v_{T}^{1 A} \leqslant 0$, or equivalently:

$$
Q\left(x^{*}, y\right)^{A}\left(\lambda r^{1}\left(x^{*}, y\right)^{A}-\psi_{T}^{L}\right) \geqslant 0 \text { for all } \lambda \in \mathbb{R} \text {. }
$$

Let $Q\left(x^{*}, y\right)_{B}$ denote the restriction of $Q\left(x^{*}, y\right)$ to rows in $B$.

Since $Q\left(x^{*}, y\right)_{B} r^{1}\left(x^{*}, y\right)>0$ we also have that:

$$
Q\left(x^{*}, y\right)_{B}\left(\lambda^{1}\left(x^{*}, y\right)-v_{T}^{1}\right) \geqslant 0 \text { for } \lambda \text { sufficiently large. }
$$

Hence $Q\left(x^{*}, y\right)\left(\lambda r^{1}\left(x^{*}, y\right)-\nu_{T}^{1}\right) \geqslant 0$ for $\lambda$ sufficiently large.

Then for $\lambda$ sufficiently large:

$$
Q\left(x^{*}, y\right)\left(\lambda r^{1}\left(x^{*}, y\right)-v_{T}^{1}\right) \geqslant 0 \text { for all } y \in Y^{p} .
$$

Likewise it can be shown that for all $\lambda$ sufficiently large:

$$
Q\left(x, y^{*}\right)\left(\lambda r^{1}\left(x, y^{*}\right)-v_{T}^{\mathbb{1}}\right) \leqslant 0 \text { for all } x \in X^{p} \text {. }
$$

Hence, if for $\lambda$ sufficiently large we look at the stochastic game $\Gamma^{*}$ defined by $r^{1 *}(s, i, j):=\lambda r^{1}(s, i, j)-v_{T}^{\prime}(s)$ and $p^{\prime \prime}(t \mid s, i, j):=p(t \mid s, i, j)$ for all $s, i, j$, then we find that the limiting average value of $\Gamma^{*}$ equals 0 and $x^{*}$ and $y^{*}$ are stationary limiting average optimal strategies in $\Gamma^{*}$. Now observe that the limiting average value of $\Gamma^{*}$ is also 0 if player 1 is restricted to mixed actions in $O_{1 s}^{*}$ for each $s \in S$, or if player 2 is restricted to mixed actions in $O_{2 s}^{*}$ for each $s \in S$. Applying theorem 5.3.1 gives the existence of $\delta_{1}$ and $\delta_{2} \in \mathbb{R}^{z}$ with:

$$
\begin{aligned}
& \delta_{1 s}=\underset{O_{1 s} \times B_{s}}{\operatorname{Val}}\left[\lambda^{1}(s, i, j)-v_{T}^{1}(s)+\sum_{i=1}^{z} p\left(t \mid s, i_{n}, j\right) \delta_{1 s}\right] \text { for each } s \in S, \\
& \delta_{2 s}=\underset{A_{1} \times O_{2}}{\operatorname{Val} l}\left[\lambda r^{1}(s, i, j)-v_{T}^{1}(s)+\sum_{i=1}^{z} p(t \mid s, i, j) \delta_{2 t}\right] \text { for each } s \in S .
\end{aligned}
$$

Using the fact that for any matrix $\left[a_{i j}\right]$ and constant $c \in \mathbb{R}$ it holds that $\operatorname{val}\left[a_{i j}+c\right]=c+\operatorname{val}\left[a_{i j}\right]$, completes the proof.

The following example and the next remark illustrate that one cannot in general take $\lambda=0$ in the above theorem. 


\subsubsection{EXAMPLE}

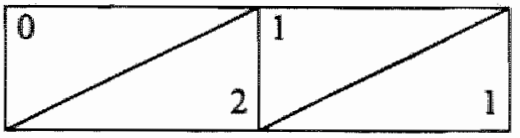

State 1

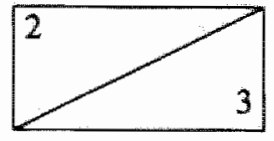

State 2

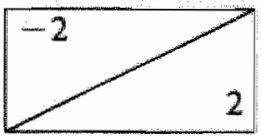

State 3

For this stochastic game $v_{T}^{1}=(1,1,-1)$.

Now consider the stochastic game $\Gamma^{*}$ as defined in the proof of above theorem. It is easy to verify that the limiting average value of $\Gamma^{*}$, with $\lambda=0$, is not equall to 0 for state 1 ; in fact it equals -1 .

Hence for the proof of the "only if'-part one cannot take $\lambda=0$ from the start.

\subsubsection{REMARK}

It should be observed that for a zero-sum stochastic game with the property that $\gamma^{1}(x, y)=0$ for all $(x, y) \in X \times Y$, one can take $\lambda=0$ in theorem 5.3.2.

\subsection{THE BAD MATCH}

In this section we examine a zero-sum stochastic game with property $P$ for which the total value does exist but for which one of the players has no history independent total c-optimal strategy. We have called this specific stochastic game "the bad match" in analogy with 'the big match' of Blackwell \& Ferguson [1968] (cf. example 1.7.4).

\subsubsection{EXAMPLE (the bad match)}

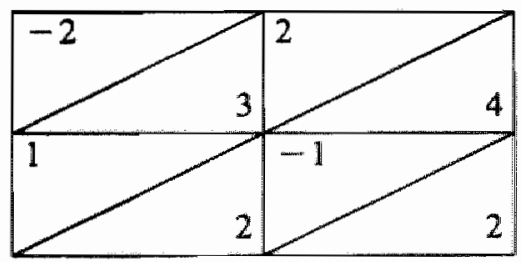

State 1

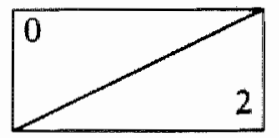

State 2

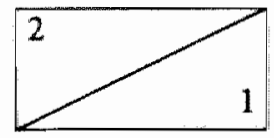

State 3

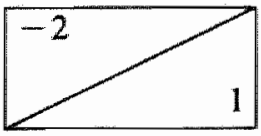

State 4

The interesting initial states are 1,3 and 4 . However, by the structure of the 
game it is clear that if we know how the players should play total (6-)optimal for initial state 1 , then the same strategies are total $(t-)$ optimal for initial states 3 and 4 . Therefore we focus on state 1 as initial state.

Observe that if the play starts in state 1 , then the players will only have to take (non-trivial) decisions at the odd stages. Those stages we call decision. epochs and strategies are determined by the mixed actions that are to be chosen on those decision epochs in state 1 . Notice that as soon as player 1 chooses action 2, then the play will move to state 2 with probability 1.

It can easily be verified that the limiting average reward is 0 for any pair of strategies and for all initial states. Hence property $P$ holds for this stochastic game.

We now define history dependent strategies for player 1 that will turn out to be total $\epsilon$-optimal for player 1 (for specific $\epsilon>0$ ).

\subsubsection{DEFINITION}

Let $p(m)=(m+1)^{-2}$ for $m \in\{0,1,2, \ldots\}$ and let $N \in \mathbb{N}$.

We define the history dependent strategy $\pi^{N}$ for player 1 by:

having observed the action choices of player 2 at the first $n$ decision epochs, say $j_{1}, j_{2}, \ldots, j_{n} \in\{1,2\}, \quad n \geqslant 0$, calculate the excess $k_{n}$ of 2 's over l's among

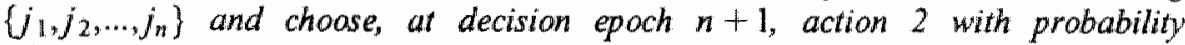
$p\left(k_{n}+N\right)$.

\subsubsection{THEOREM}

a) The total value of the bad match exists and equals 0 (for initial state ().

b) For player 2 a stationary total optimal strategy is to use the mixed action $(1 / 2,1 / 2)$ in state 1 at all decision epochs.

c) The strategy $\pi^{N}$ is total $(N+1)^{-1}$-optimal for player 1 , for all $N \in \mathbb{N}$.

d) Player 1 has no history independent total e-optimal strategy for $\epsilon>0$ sufficiently small.

e) Player I has no total optimal strategy.

This theorem follows directly from 5.4.4 - 5.4.15 below.

\subsubsection{LEMMA}

Let $y^{*}$ be the stationary strategy for player 2 defined by using $(1 / 2,1 / 2)$ in state 1 at all decision epochs. Then $\gamma_{T}^{l}\left(1, \pi, y^{*}\right)=0$ for each $\pi \in \Pi$.

\section{PRoOF:}

Whatever actions player 1 chooses, at each stage the expected payoff will be 0 if player 2 uses $y^{*}$. Namely, in state 1 at each decision epoch the expected payoff is 0 ; at other stages the play is either in state 2 with payoff 0 or the play is in state 3 or state 4 with the same probability, giving allso expected payoff 0.

The following corollary is immediate. 


\subsubsection{Corollary}

$$
\inf _{\sigma} \sup _{\pi} \gamma_{T}^{1}(1, \pi, \sigma)<0 .
$$

\subsubsection{LEMMA}

With Markov strategies player I cannot guarantee a total reward larger than -1 .

\section{Proof:}

Let $f$ be a Markov strategy for player 1. We consider two cases:

a) Suppose that the probability that player 1 will ever choose action 2 is 0 . Then player 1 chooses action 1 at all decision epochs with probability 1 . The stationary strategy $y^{1}:=(1,0)$ for player 2 leads to $\gamma_{T}^{1}\left(1, f, y^{1}\right)=-1$.

b) Suppose that the probability that player 1 will ever choose action 2 is $\epsilon>0$. Then for each $\delta \in(0, \epsilon)$ there is an $N_{\delta} \in \mathbb{N}$ such that the probability of player 1 choosing action 2 before stage $N_{\delta}$, is larger that $\epsilon-\delta$. For each $\delta \in(0, \epsilon)$ define strategy $g^{\delta}$ for player 2 by: at decision epochs $1,2, \ldots, N_{\delta}$ choose action 2 and at all other decision epochs choose action 1 . Then we have that:

$$
\gamma_{T}^{1}\left(1, f, g^{\delta}\right) \leqslant(\epsilon-\delta) \cdot(-1)+\delta \cdot(1)+(1-\epsilon) \cdot(-1)=-1+2 \delta
$$

Since player 2 can choose $\delta$ arbitrarily small, the proof is complete.

The next lemma says that player 1 has no limiting average optimal strategy.

\subsubsection{LEMMA}

For any strategy $\pi$ for player $l$ there is some $\sigma$ such that $\gamma_{T}^{1}(1, \pi, \sigma)<0$.

\section{Proof:}

Let $\pi$ be a strategy for player 1 . If there is no sequence of action choices of player 2 , such that player 1 chooses action 2 with positive probability, then it is clear that $\gamma_{T}^{1}\left(1, \pi, y^{1}\right)=-1$ for the stationary strategy $y^{1}:=(1,0)$.

So suppose there is some sequence of action choices $\left(j_{1}, j_{2}, \ldots, j_{m}\right)$ such that at decision epoch $m+1$ player 1 will, for the first time, choose action 2 with positive probability $>0$. Now define $g^{m}$ for player 2 by: at decision epochs $n \leqslant m$ choose $j_{n}$ with probability 1 , at decision epoch $m+1$ choose action 2 with probability 1 , at decision epochs $n>m+1$ use the mixed action $(1 / 2,1 / 2)$. It can be verified that $\gamma_{T}^{1}\left(1, \pi, g^{m}\right)=-\varepsilon<0$.

We will now show that $\gamma_{T}^{\frac{1}{T}}\left(1, \pi^{N}, \sigma\right) \geqslant-(N+1)^{-1}$ for all $a \in \Sigma$, where $\pi^{N}$ is the strategy as defined in definition 5.4.2.

In order to prove this, we fix an arbitrary strategy $\sigma \in \Sigma$ and we define several random variables which are supposed to correspond to the pair of strategies $\left(\pi^{N}, \sigma\right)$ 


\subsubsection{DeFINITION}

Let $\sigma \in \Sigma$. Suppose that the players use $\left(\pi^{N}, \sigma\right)$ for some $N \in \mathbb{N}$.

Let $B$ (Bottom) be the random variable denoting the number of decision epochs before player I chooses action 2.

For each $m \in \mathbb{N}$ define the event $K(m)$ by:

$K(m):=\left\{B \geq m\right.$, or $B<m$ and $\left.j_{B+1}=1\right\}$.

Let $P_{N}\{K(m)\}$ be the probability that $K(m)$ occurs.

Notice that $K(m)$ is the event that at decision epoch $m$ player 1 either has not yet chosen action 2 , or he did choose action 2 and was lucky in receiving 1 . In other words we have that $K(m)$ is the event that the total reward up to decision epoch $m$ is non-negative.

\subsubsection{REMARK}

$$
\begin{aligned}
P_{N}\{K(m+1)\}= & P_{N}\left\{B=0 \text { and } j_{1}=1\right\} \\
& +P_{M}\left\{B \geqslant m+1 \text {, or } 1 \leqslant B<m+1 \text { and } j_{B+1}=1 j_{1}=1\right\} \\
& +P_{M}\left\{B \geq m+1 \text {, or } 1 \leqslant B<m+1 \text { and } j_{B+1}=1 j_{1}=2\right\} .
\end{aligned}
$$

\subsubsection{LEMMA}

a) $P_{N}\left\{B \geqslant m+1\right.$, or $1 \leqslant B<m+1$ and $\left.j_{B+1}=1 \mid j_{1}=1\right\}$

$=(1-p(N)) P_{N-1}\{K(m)\}$.

b) $P_{N}\left(B \geqslant m+1\right.$, or $\mathbb{1} \leqslant B<m+1$ and $\left.j_{B+1}=1 \mid j_{1}=2\right\}$

$=(1-p(N)) P_{N+1}\{K(m)\}$.

\section{Proof:}

We only prove (a) since the proof of (b) is similar.

Notice that in the left-hand side of (a) the event $B=0$ is excluded. Given that $j_{1}=1$, the mixed action to be used according to $\pi^{N}$ at decision epoch $n+1$, with some history $\left(1, j_{2}, j_{3}, \ldots, j_{n}\right)$, is equal to the mixed action used according to $\pi^{N-1}$ at decision epoch $n$ with history $\left(j_{2}, j_{3}, \ldots, j_{n}\right)$.

At decision epoch 1 player 1 using $\pi^{N}$ chooses action 1 with probability $1-p(N)$.

Hence, given that $j_{1}=1$, using $\pi^{N}$ yields the same stochastic process as initially choosing action $\mathbb{1}$ with probability $1-p(N)$ and using $\pi^{N-1}$ thereafter.

Consequently, we have that

$$
\begin{aligned}
P_{N}\{K(m+1)\}= & P_{N}\left\{B=0 \text { and } j_{1}=1\right\} \\
& +(1-p(N)) P_{N-1}\{K(m)\}+(1-p(N)) P_{N+1}\{K(m)\} .
\end{aligned}
$$

The next lemma states that for all $m$ and $N$ the probability that the total reward up to decision epoch $m$ is non-negative, is at least $N / 2(N+1)$. 


\subsubsection{LEMMA}

$$
P_{N}\{K(m)\} \geqslant N / 2(N+1) \text { for all } m, N \in \mathbb{N}
$$

\section{ProOF :}

We use induction to $m$.

a) Let $m=1$ and let $N \in \mathbb{N}$.

If $j_{1}=1$, then:

$P_{N}\left\{B \geqslant 1 j_{1}=1\right\}=1-p(N)$ and $P_{N}\left(B<1\right.$ and $\left.j_{B+1}=1 j_{1}=1\right\}=p(N)$.

So $P_{N}\left\{K(1) j_{1}=1\right)=1>N / 2(N+1)$.

If $j_{1}=2$, then:

$P_{N}\left\{K(1) j_{i}=2\right\}=P_{N}\left\{B \geqslant 1 j_{1}=2\right\}=1-p(N) \geqslant N / 2(N+1)$.

Let $q$ be the probability that player 2 chooses action 1 at decision epoch 1, then:

$$
\begin{aligned}
P_{N}\{K(1)\} & =q P_{N}\left\{K(1) j_{1}=1\right\}+(1-q) P_{N}\left\{K(1) j_{1}=2\right\} \\
& \geqslant q N / 2(N+1)+(1-q) N / 2(N+1)=N / 2(N+1) .
\end{aligned}
$$

b) Suppose $P_{N}(K(m)\}>N / 2(N+1)$ for some $m \in \mathbb{N}$ and all $N \in \mathbb{N}$.

Then, in view of remark 5.4.9 and lemma 5.4.10, we have:

$$
\begin{aligned}
P_{N}\left\{K(m+1) j_{1}=1\right\}= & P_{N}\left\{B=0 \text { and } j_{1}=1 j_{1}=1\right\} \\
& +P_{N}\{B \geqslant m+1, \text { or } 1 \leqslant B<m+1 \\
& \text { and } \left.j_{B+1}=1 j_{1}=1\right\} \\
= & p(N)+(1-p(N)) P_{N-1}\{K(m)\} \\
\geqslant & p(N)+(1-p(N))(N-1) / 2 N=N / 2(N+1) .
\end{aligned}
$$

Also in view of lemma 5.4.10 we have:

$$
\begin{aligned}
& \begin{aligned}
P_{N}\left\{K(m+1) j_{1}=2\right\}= & P_{N}\left\{B=0 \text { and } j_{1}=1 \mid j_{1}=2\right\} \\
& +P_{N}\{B \geqslant m+1 \text {, or } 1 \leqslant B<m+1 \\
& \text { and } \left.j_{B+1}=1 j_{1}=2\right\} \\
= & +(1-p(N)) P_{N+1}\{K(m)\} \\
> & (1-P(N))(N+1) / 2(N+2)=N / 2(N+1) . \\
\text { Hence } P_{N}(K(m+1)\}= & q P_{N}\left(K(m+1) \mid j_{1}=1\right\} \\
& +(1-q) P_{N}\left\{K(m+1) j_{1}=2\right\} \geq N / 2(N+1),
\end{aligned} \\
& \text { which shows the induction step. }
\end{aligned}
$$

The following lemma demonstrates that $\pi^{N}$ guarantees a total reward of at least $-(N+1)^{-1}$ if the probability that player 1 will ever choose action 2 , using $\pi^{N}$ against $\sigma$, is 1 .

\subsubsection{LEMMA}

If $\lim _{m \rightarrow \infty} P_{N}(B \geqslant m)=0$, then $\gamma_{T}^{1}\left(1, \pi^{N}, \sigma\right) \geqslant-(N+1)^{-1}$.

\section{Proof:}

Since by definition $P_{N}\{K(m)\}=P_{N}(B \geqslant m\}+P_{N}\left\{B<m\right.$ and $\left.j_{B+1}=1\right\}$, we derive from lemma 5.4.11, in view of the assumption of this lemma, that: 


$$
\lim _{m \rightarrow \infty} P_{N}\{K(m)\}=P_{N}\left[j_{B+1}=1\right\} \geqslant N / 2(N+1) .
$$

With probability 1 player 1 will choose action 2 at some decision epoch and, since the sum of payoffs until that decision epoch equals 0 , the total reward is determined by the action which player 2 chooses at that moment. So we have:

$$
\begin{aligned}
\gamma_{T}^{1}\left(1, \pi^{N}, \sigma\right) & =P_{N}\left(j_{B+1}=1\right\}-P_{N}\left\{j_{B+1}=2\right\} \\
& =2 P_{N}\left\{j_{B+1}=1\right\}-1 \\
& \geqslant N /(N+1)-1=-(N+1)^{-1} .
\end{aligned}
$$

Notice that, if for a certain $n$ we have $k_{n}=-N$, then player 1 will choose action 2 with probability 1 at decision epoch $n+1$. Hence, as long as no transition to state 2 has occurred, we have $k_{n} \geqslant-N$.

\subsubsection{LEMMA}

For any realization of the stochastic process associated to $\pi^{N}$ and $\sigma$, for which player 1 never chooses action 2, it holds that the corresponding total reward is at least 0 .

\section{Proof:}

Let $\left(r_{1}, r_{2}, \ldots\right)$ be the sequence of payoffs (to player 1$)$ that occurs.

Since in this case $k_{n}>-N$ for all $n \in \mathbb{N}$, we have $\sum_{t=1}^{T} \sum_{n=1}^{t} r_{n}>-2 N$ for every $T \in \mathbb{N}$. It follows that $\liminf _{T \rightarrow \infty} \frac{1}{T} \sum_{t=1}^{T} \sum_{n=1}^{t} r_{n} \geqslant 0$.

\subsubsection{LEMMA}

If $\lim _{m \rightarrow \infty} P_{N}(B \geqslant m\}>0$, then $\gamma \frac{1}{T}\left(1, \pi^{N}, \sigma\right) \geqslant-(N+1)^{-1}$.

\section{Proof:}

For $m \in \mathbb{N}$ let $\lambda(m):=P_{N}\left\{B<m\right.$ and $\left.j_{B+1}=1\right\}$ and let $\mu(m):=P_{N}\{B<m$ and $\left.j_{B+1}=2\right\}$. Since $\{\lambda(m): m \in \mathbb{N}\}$ and $\{\mu(m): m \in \mathbb{N}\}$ are bounded monotone increasing sequences, we can define $\lambda:=\lim _{m \rightarrow \infty} \lambda(m)$ and $\mu:=\lim _{m \rightarrow \infty} \mu(m)$.

Now the probability that player 1 will ever choose action 2 , equals $\lambda+\mu$ and hence $1-\lambda-\mu$ is the probability that the play never reaches state 2 . By lemma 5.4.13 and by the definitions of $\lambda$ and $\mu$ we have:

$$
\gamma_{T}^{1}\left(1, \pi^{N}, 0\right) \geqslant \lambda \cdot 1+\mu \cdot(-1)+(1-\lambda-\mu) \cdot 0=\lambda-\mu .
$$

So if we can prove that $\lambda-\mu>-(N+1)^{-1}$, then the proof is finished.

For each $m \in \mathbb{N}$ define strategy $\sigma^{m}$ by: up to decision epoch $m$ use $\sigma$, at all other decision epochs use the mixed action $(1 / 2,1 / 2)$. Then $\sigma^{m}$ will give rise to sequences $\left(j_{1}, j_{2}, \ldots\right)$ for which $\left[k_{n}=-N\right.$ for some $\left.n \in \mathbb{N}\right]$ with probability 1 . Hence for the strategies $\sigma^{m}$ the condition of lemma 5.4.12 applies (where $P_{N}$ now refers to $\left.\left(\pi^{N}, \sigma^{m}\right)\right)$. Hence $\gamma_{T}^{1}\left(1, \pi^{N}, \sigma^{m}\right) \geqslant-(N+1)^{-1}$ for all $m \in \mathbb{N}$. 
On the other hand, with respect to $\left(\pi^{N}, \sigma^{m}\right)$, if player 1 chooses action 2 before decision epoch $m$, then this contribures $\lambda(m) \cdot 1+\mu(m) \cdot(-1)$ to $\gamma_{T}^{1}\left(1, \pi^{N}, \sigma^{m}\right)$, and choosing action 2 later contributes $(1-\lambda(m)-\mu(m)) \cdot 0$ (cf. lemma 5.4.4).

Hence $\gamma h\left(1, \pi^{N}, \sigma^{m}\right)=\lambda(m)-\mu(m) \geqslant-(N+1)^{-1}$ for all $m \in \mathbb{N}$. Taking limits for $m$ to $\infty$ gives $\lambda-\mu \geqslant-(N+1)^{-1}$, which completes this proof.

An immediate consequence of lemma 5.4.12 and lemma 5.4.14 is the following.

\subsubsection{Corollary}

$$
\sup _{\pi} \inf _{\sigma} \gamma_{T}^{l}(1, \pi, \sigma) \geqslant 0
$$

It should be remarked that the above proofs for the bad match are along the same lines as the proofs given by Blackwell \& Ferguson [1968] for the big match.

\subsection{Conclusions}

The bad match illustrates that there is an analogy between the total reward criterion and the limiting average reward criterion. For both criteria history dependent strategies are indispensable for playing e-optimal, which distinguishes these criteria from the $\beta$-discounted reward criterion. The relation between the total reward criterion and the limiting average reward criterion is even narrowed by the fact that with any stochastic game $\Gamma$ we can relate another stochastic game $\Gamma^{*}$ with an infinite state space, such that for all strategies the total reward in $\Gamma$ equals the related limiting average reward in $\Gamma^{*}$. To show this, let $T$ be a zero-sum stochastic game as in definition 1.2.1 and let $H_{n}, n \in \mathbb{N}$, be as defined in definition 1.3.3. We use asteriks to define $\Gamma^{*}$.

Let $S^{*}:=\bigcup_{n=1}^{\infty} H_{n}$ be the state space of $\Gamma^{*}$.

For $s^{*}=h_{n}=\left(s_{1}, i_{1}, j_{1}, s_{2}, i_{2}, j_{2}, \ldots, s_{n-1}, i_{n-1}, j_{n-1}, s_{n}\right) \quad$ let $A_{s}^{*}:=A_{s_{n}} \quad$ and $B_{s}^{*}:=B_{s_{a}}$ be the action spaces in $\Gamma^{*}$.

For $s^{*}=h_{n}$ and $i^{*} \in A_{s}^{*}, j^{*} \in B_{s}$ let $r^{1 *}\left(s^{*}, i^{*}, j^{*}\right):=\sum_{k=1}^{n-1} r^{1}\left(s_{k}, i_{k}, j_{k}\right)$ $+r^{\prime}\left(s_{n}, i^{*}, j^{*}\right)$ and let $p^{*}\left(t^{*} \mid s^{*}, i^{*}, j^{*}\right):=p\left(t \mid s_{n}, i^{*}, j^{*}\right)$ for $t^{*}=\left(h_{n}, i^{*}, j^{*}, t\right)$ and $p^{*}\left(t^{*} \mid s^{*}, i^{*}, j^{*}\right):=0$ for other $t^{*} \in S^{*}$.

Hence we have translated histories of $\Gamma$ into states in $\Gamma^{*}$. Notice that in $\Gamma^{*}$ every state $s^{*}$ can be reached along precisely one history path, and there is a one-to-one relation between strategies in $\Gamma$ and strategy classes in $\Gamma^{*}$.

Furthermore we have that at each stage $N \in \mathbb{N}$, for strategies $\pi, \sigma$ and initial state $s^{*}=s$, it holds that:

$$
\sum_{m=1}^{N} E_{s * n}\left[R^{\prime^{*}}(m)\right]=\sum_{m=1}^{N} E_{s \pi \sigma}\left[\sum_{n=1}^{m} R^{1}(n)\right]=\sum_{m=1}^{N} \sum_{n=1}^{m} E_{s \pi \sigma}\left[R^{1}(n)\right] .
$$

Hence $\gamma^{1^{*}}\left(s^{*}, \pi, \sigma\right)=\gamma_{T}^{\mathrm{l}}(s, \pi, \sigma)$ for all $s^{*}=s$, and all strategies $\pi$ and $\sigma$. 



\section{Chapter 6}

\section{Stochastic games and mathematical programming}

\subsection{INTRODUCTION}

Mangasarian \& Stone [1964] proved the following theorem which relates equilibria of a bimatrix game with solutions of an associated non-linear program, with quadratic objective function and with linear constraints.

\subsubsection{THEOREM}

For a bimatrix game $\left(A^{1}, A^{2}\right)$ a pair of mixed actions $\left(x^{*}, y^{*}\right)$ is an equilibrium with payoffs $\left(\alpha^{1^{*}}, \alpha^{2^{*}}\right)$ if and only if $\left(x^{*}, y^{*}, \alpha^{1 *}, \alpha^{2^{*}}\right)$ is a global minimum in the following non-linear program, with objective value 0 .

NLP 6.1.1:

variables $x \in \mathbb{R}^{m}, y \in \mathbb{R}^{n}, \alpha^{1}, \alpha^{2} \in \mathbb{R}$

minimize $\alpha^{1}-x A^{1} y+\alpha^{2}-x A^{2} y$

subject to $\alpha^{1} 1_{m}-A^{1} y \geq 0$ and $\alpha^{2} 1_{m}-x A^{2} \geqslant 0$

$$
\begin{aligned}
& \sum_{i=1}^{m} x_{i}=1 \text { and } \sum_{j=1}^{n} y_{j}=1 \\
& x \geq 0 \text { and } y \geq 0 .
\end{aligned}
$$

For matrix games $A$, where player 1 is the maximizing player, the non-linear factors in the objective function of NLP 6.1.1 disappear (since their sum is 0 ) and what remains is a linear program. It is easy to verify that for matrix games we have the following resullt.

\subsubsection{THEOREM}

For an $m \times n$ matrix game $A$ the value, for player 1 , is $v$ and $\left(x^{*}, y^{*}\right)$ are optimal mixed actions for the players, if and only if $\left(x^{*}, y^{*}, v,-v\right)$ is a global minimum in the following linear program, with objective value 0 .

LP 6.1.2:

variables $x \in \mathbb{R}^{m}, y \in \mathbb{R}^{n}, \alpha^{1}, \alpha^{2} \in \mathbb{R}$

minimize $\alpha^{1}+\alpha^{2}$

subject to $\alpha^{1} 1_{m}-A y>0$ and $\alpha^{2} 1_{n}+x A \geqslant 0$

$$
\begin{aligned}
& \sum_{i=1}^{m} x_{i}=1 \text { and } \sum_{j=1}^{n} y_{j}=1 \\
& x \geqslant 0 \text { and } y \geqslant 0 .
\end{aligned}
$$


In this chapter we will formulate analogues of the above theorems for stochastic games. We consider both the general-sum and the zero-sum case for the $\beta$ discounted reward criterion, for the limiting average reward criterion and for the total reward criterion. For the last criterion we have restricted our attention to stochastic games with the property that the limiting average reward is 0 for all pairs of stationary strategies. For the $\beta$-discounted criterion and the limiting average criterion, characterizations for stationary solutions by means of mathematical programs have been reported in Rogers [1969], Rothblum [1979], Hordijk \& Kallenberg [1981], Vrieze [1981, 1983, 1987-a], Filar [1986], Filar \& Schultz [1986, 1987], Schultz [1987]. However several of these characterizations are for special classes of stochastic games and/or for the zero-sum case only.

Since it is well-known that only with respect to the $\beta$-discounted reward criterion stationary optimal strategies and stationary equilibria always exist, it is of interest to know, especially for the other criteria, whether near-optimal solutions of the programs correspond with $\epsilon$-optimal strategies or $\epsilon$-equilibria. For the $\beta$-discounted reward criterion this is indeed the case. However for the limiting average reward criterion and for the total reward criterion this correspondence between near-optimal solutions not necessarily holds. Nevertheless, for the zero-sum case the program we formulate will for both players lead to stationary ( $\mathrm{c}$-)optimal strategies, whenever they exist. If stationary $\epsilon$-optimal strategies fail to exist, then our program finds ' $\mathrm{e}$-best' stationary strategies for both players. Here an 'e-best' stationary strategy for player 1 is a strategy $x_{\varepsilon}$ such that $\inf _{y \in Y} \gamma^{1}\left(x_{\epsilon}, y\right)+\epsilon \geqslant \sup _{x \in X \in Y} \inf _{y \in Y} \gamma^{1}(x, y)$.

The programs we present are based on the lemmas 1.5.3 to 1.5.8 and on lemma 1.6.2, as well as on theorems 1.7.3, 5.3.1 and 5.3.2. The results of the sections 6.2 and 6.3 can be found in Filar et al. [1987].

\subsection{PRograms Fon THE $\beta$-DisCOUNTED REWARD CRITERION}

\subsubsection{LEMMA}

For a general-sum stochastic game a pair of stationary strategies $\left(x^{*}, y^{*}\right)$ is a $\beta$ discounted equilibrium with $\beta$-discounted rewards $\left(\alpha^{1^{*}}, \alpha^{2 *}\right)$ if and only if for all $s \in S$ and $k \in\{1,2\}$ :

a) $\alpha^{k^{*}}=(1-\beta) r^{k}\left(s, x_{s}^{*}, y_{s}^{*}\right)+\beta \sum_{i=1}^{z} p\left(t \mid s, x_{s}^{*}, y_{s}^{*}\right) \alpha_{i}^{k^{*}}$

b) $\alpha_{s}^{1^{*}} \geq(1-\beta) r^{1}\left(s, i, y_{s}^{*}\right)+\beta \sum_{i=1}^{z} p\left(t \mid s, i, y_{s}^{*}\right) \alpha_{i}^{1^{*}}$ for all $i \in A_{s}$

$$
\alpha_{s}^{2^{*}} \geq(1-\beta) r^{2}\left(s, x_{s}^{*}, j\right)+\beta \sum_{t=1}^{*} p\left(t \mid s_{,}, x_{s}^{*}, j\right) \alpha_{i}^{2^{*}} \text { for all } j \in B_{s} \text {. }
$$

\section{Proof:}

This lemma follows directly from lenma 1.5.3, lemma 1.6.2 and lemma 1.6.4. 
From lemma 6.2.1 we immediately obtain the next theorem.

\subsubsection{THEOREM}

For a general-sum stochastic game a pair of stationary strategies $\left(x^{*}, y^{*}\right)$ is a $\beta$ discounted equilibrium with $\beta$-discounted rewards $\left(\alpha^{1{ }^{*}}, \alpha^{2^{2}}\right)$ if and only if $\left(x^{*}, y^{*}, \alpha^{1^{*}}, \alpha^{2^{*}}\right)$ is a global minimum in the following non-linear program, with objective value 0 .

NLP 6.2.2:

variables $\quad x \in \underset{s=1}{\sum_{s}} \mathbb{R}^{m_{s}}, y \in \underset{s=1}{\sum_{x=1}^{*}} \mathbb{R}^{n_{x}}, \alpha^{1}, \alpha^{2} \in \mathbb{R}^{z}$

minimize $\sum_{k=1}^{2^{s}} \sum_{s=1}^{z}\left(\alpha_{s}^{k}-(1-\beta) r^{k}\left(s, x_{s}, y_{s}\right)-\beta \sum_{i=1}^{z} p\left(t \mid s, x_{s}, y_{s}\right) \alpha_{i}^{k}\right)$

stubject to

a) $\quad \alpha_{s}^{1} \geqslant(1-\beta) r^{1}\left(s, i, y_{s}\right)+\beta \sum_{t=1}^{z} p\left(t \mid s, i, y_{s}\right) \alpha_{i}^{1}$ for all $i \in A_{s}, s \in S$

$$
\alpha_{s}^{2} \geqslant(1-\beta) r^{2}\left(s, x_{s}, j\right)+\beta \sum_{i=1}^{z} p\left(t \mid s, x_{s}, j\right) \alpha_{t}^{2} \text { for all } j \in B_{s}, s \in S
$$

b) $\sum_{i=1}^{m_{s}} x_{s}(i)=1$ and $\sum_{j=1}^{n_{s}} y_{s}(j)=1$ for all $s \in S$

c) $x_{s} \geqslant 0$ and $y_{s} \geqslant 0$ for all $s \in S$.

An interesting feature of the above non-linear program is that feasible solutions with objective value near 0 are directly related with stationary $\beta$ discounted $\epsilon$-equilibria.

\subsubsection{COROLLARY}

If $\left(x^{*}, y^{*}, \alpha^{1^{*}}, \alpha^{2 *}\right)$ is a feasible solution of NLP 6.2 .2 with objective value $\delta>0$, then $\left(x^{*}, y^{*}\right)$ is a stationary $\beta$-discounted $\delta(1-\beta)^{-1}$-equilibrium.

Proof:

Constraints (b) and (c) give that $x^{*} \in X$ and $y^{*} \in Y$.

By the constraints (a) and by the objective value $\delta>0$ for the solution $\left(x^{*}, y^{*}, \alpha^{1 *}, \alpha^{2^{*}}\right)$ we have for each $s \in S$ :

$$
0 \leqslant \alpha_{s}^{k^{*}}-(1-\beta) r^{k}\left(s, x_{s}^{*}, y_{s}^{*}\right)-\beta \sum_{i=1}^{*} p\left(t \mid s, x_{s}^{*}, y_{s}^{*}\right) \alpha_{i}^{k^{*}} \leqslant \delta \text {. }
$$

Or, equivalently, in vector notation:

$$
0 \leqslant \alpha^{k^{*}}-(1-\beta) r^{k}\left(x^{*}, y^{*}\right)-\beta P\left(x^{*}, y^{*}\right) \alpha^{k^{*}} \leqslant \delta 1_{z} .
$$

By the first inequality sign: $\alpha^{k^{*}} \geqslant \gamma_{\beta}^{k}\left(x^{*}, y^{*}\right)$ (cf. lemma 1.5.4).

The second inequality sign gives us:

$$
\left(I-\beta P\left(x^{*}, y^{*}\right)\right) \alpha^{k^{*}} \leqslant(1-\beta) r^{k}\left(x^{*}, y^{*}\right)+\delta 1_{z},
$$

and hence $\alpha^{k^{*}} \leqslant(1-\beta)\left(I-\beta P\left(x^{*}, y^{*}\right)\right)^{-1} r^{k}\left(x^{*}, y^{*}\right)+\left(I-\beta P\left(x^{*}, y^{*}\right)\right)^{-1} \delta 1_{z}$

$$
=\gamma_{\beta}^{k}\left(x^{*}, y^{*}\right)+\delta(1-\beta)^{-1} 1_{z} \text {. }
$$


Constraints (a) also imply:

$$
\gamma_{\beta}^{l}\left(x, y^{*}\right) \leqslant \alpha^{1^{*}} \text { for all } x \in X \text { and } \gamma_{\beta}^{2}\left(x^{*}, y\right) \leqslant \alpha^{2^{*}} \text { for all } y \in Y \text {. }
$$

Combining these results proves this corollary.

Of course, the reduction of NLP 6.2 .2 to zero-sum stochastic games is a program which finds the $\beta$-discounted value and stationary optimal strategies for both players. Rothblum [1979] proposed the following non-linear program to find the $\beta$-discounted value and a stationary $\beta$-discounted optimal strategy for player 2 .

\subsubsection{THEOREM}

For a zero-sum stochastic game with $\beta$-discounted value $v_{\beta}^{1}$ a stationary strategy $y^{*} \in Y$ is $\beta$-discounted optimal for player 2 if and only if $\left(y^{*}, v_{\beta}^{1}\right)$ is a global minimum in the following non-linear program.

NLP 6.2.4:

variables $y \in \stackrel{*}{\times} \mathbb{R}^{n_{\xi}}, \alpha \in \mathbb{R}^{z}$

minimize $\sum_{s=1}^{z} \alpha_{s}$

subject to
a) $\alpha_{s} \geq(1-\beta) r^{1}\left(s, i, y_{s}\right)+\beta \sum_{t=1}^{z} p\left(t \mid s, i, y_{s}\right) \alpha_{t}$ for all $i \in A_{s}, s \in S$
b) $\sum_{j=1}^{n_{s}} y_{s}(j)=1$ for all $s \in S$
c) $y_{s} \geqslant 0$ for all $s \in S$.

It is obvious that a similar program can be formulated to find stationary $\beta$ discounted optimal strategies for player 1.

\subsection{Programs for THe LIMITING AVERAGE REWARD CRUTERION}

It is well-known that stationary limiting average equilibria may fail to exist. However, below we present a non-linear program which finds a stationary limiting average equilibrium whenever one exists. Our program is based on the following lemma.

\subsubsection{LEMMA}

A pair of stationary strategies $\left(x^{*}, y^{*}\right)$ is a limiting average equilibrium with limiting average rewards $\left(\alpha^{1^{*}}, \alpha^{2^{*}}\right)$ if and only if there exist $\delta^{1^{*}}, \delta^{2^{2}}, \mu^{1^{*}}, \mu^{2^{*}} \in \mathbb{R}^{z}$ with:
a) $\alpha_{s}^{k^{*}}=\sum_{t=1}^{z} p\left(t \mid s, x_{s}^{*}, y_{s}^{*}\right) \alpha_{t}^{k^{*}}$ for all $s \in S, k \in\{1,2\}$
b) $\alpha_{s}^{k^{*}}+s_{s}^{k^{*}}=r^{k}\left(s, x_{s}^{*}, y_{s}^{*}\right)+\sum_{t=1}^{z} p\left(t \mid s, x_{s}^{*}, y_{s}^{*}\right) \delta_{t}^{k^{*}}$ for all $s \in S, k \in\{1,2\}$ 
$\begin{aligned} & \text { c) } \alpha_{s}^{1^{*}} \geqslant \sum_{i=1}^{z} p\left(t \mid s, i, y_{s}^{*}\right) \alpha_{i}^{1^{*}} \text { for all } i \in A_{s}, s \in S \\ & \alpha_{s}^{2^{*}} \geqslant \sum_{t=1}^{z} p\left(t \mid s, x_{s}^{*}, j\right) \alpha_{s}^{2^{*}} \text { for all } j \in B_{s}, s \in S \\ & \text { d) } \quad \alpha_{s}^{1^{*}}+\mu_{s}^{l^{*}} \geqslant r^{1}\left(s, i, y_{s}^{*}\right)+\sum_{i=1}^{z} p\left(t \mid s, i, y_{s}^{*}\right) \mu_{t}^{1^{*}} \text { for all } i \in A_{s}, s \in S \\ & \alpha_{s}^{2^{*}}+\mu_{s}^{2^{*}} \geqslant r^{2}\left(s, x_{s}^{*}, j\right)+\sum_{t=1}^{z} p\left(t \mid s, x_{s}^{*}, j\right) \mu_{i}^{2^{*}} \text { for all } j \in B_{s}, s \in S .\end{aligned}$

This lemma follows from the fact that $\left(x^{*}, y^{*}\right)$ is a limiting average equilibrium if and only if $x^{*}$ is limiting average optimal for player 1 in $\operatorname{MDP}\left(y^{*}\right)$ and $y^{*}$ is limiting average optimal for player 2 in $\mathrm{MDP}\left(x^{*}\right)$ (cf. section 1.6). A stationary strategy $x^{*}$ is limiting average optimal in MDP( $\left.y^{*}\right)$ with limiting average reward $\alpha^{1 *}$ if and only if there exist $\delta^{1 *}$ and $\mu^{1^{*}}$ such that (cf. Blackwell [1962], Hordijk \& Kallenberg [1979]):
a) $\alpha_{s}^{1^{*}}=\sum_{t=1}^{n} p\left(t \mid s, x_{s}^{*}, y_{s}^{*}\right) \alpha_{t}^{l^{*}}$ for all $s \in S$
b) $\alpha_{s}^{1^{*}}+\delta_{s}^{1^{*}}=r^{1}\left(s_{y} x_{s}^{*}, y_{s}^{*}\right)+\sum_{t=1}^{z} p\left(t \mid s, x_{s}^{*}, y_{s}^{*}\right) \delta_{t}^{1^{*}}$ for all $s \in S$
c) $\alpha_{s}^{1^{*}} \geq \sum_{t=1}^{z} p\left(t \mid s, i, y_{s}^{*}\right) \alpha_{i}^{1^{*}}$ for all $i \in A_{s}, s \in S$
d) $\alpha_{s}^{1^{*}}+\mu_{s}^{1^{*}} \geqslant r^{1}\left(s, i, y_{s}^{*}\right)+\sum_{i=1}^{*} p\left(t \mid s, i, y_{s}^{*}\right) \mu_{i}^{1^{*}}$ for all $i \in A_{s}, s \in S$.

It is easy to verify that lemma 6.3.1 directly implies the following result.

\subsubsection{THEOREM}

For a general-sum stochastic game a pair of stationary strategies $\left(x^{*}, y^{*}\right)$ is a limiting average equilibrium with limiting average rewards $\left(\alpha^{1^{*}}, \alpha^{2^{*}}\right)$ if and only if there exist $\delta^{1^{*}}, \delta^{2^{*}}, \mu^{1^{*}}, \mu^{2^{*}} \in \mathbb{R}^{z}$ such that $\left(x^{*}, y^{*}, \alpha^{1^{*}}, \alpha^{2^{*}}, \delta^{1^{*}}, \delta^{2^{*}}, \mu^{1^{*}}, \mu^{2^{*}}\right)$ is a global minimum in the following non-linear program, with objective value 0 .

NLP 6.3.2:

variables $x \in \stackrel{z}{\times} \mathbb{R}^{m_{3}}, y \in \stackrel{\ddot{x}}{\times} \mathbb{R}^{n_{*}}, \alpha^{1}, \alpha^{2}, \delta^{1}, \delta^{2}, \mu^{1}, \mu^{2} \in \mathbf{R}^{z}$

minimize $\sum_{k=1}^{2} \sum_{s=1}^{z}\left[\alpha_{s}^{k}-\sum_{t=1}^{z} p\left(t \mid s, x_{s}, y_{s}\right) \alpha_{t}^{k}\right]$

subject to
a) $\alpha_{s}^{1} \geqslant \sum_{i=1}^{z} p\left(t \mid s_{x} i_{i} y_{s}\right) \alpha_{i}^{1}$ for ull $i \in A_{s}, s \in S$ $\alpha_{s}^{2} \geqslant \sum_{i=1}^{z} p\left(t \mid s, x_{s}, j\right) \alpha_{t}^{2}$ for all $j \in B_{s}, s \in S$
b) $\alpha_{s}^{1}+\delta_{s}^{1}=r^{1}\left(s, x_{s}, y_{s}\right)+\sum_{i=1}^{n} p\left(t \mid s, x_{s}, y_{s}\right) \delta_{l}^{l}$ for all $s \in S$ $\alpha_{s}^{2}+\delta_{s}^{2}=r^{2}\left(s, x_{s}, y_{s}\right)+\sum_{t}^{z} p\left(r \mid s, x_{s}, y_{s}\right) \delta_{t}^{2}$ for all $s \in S$ 
c) $\alpha_{s}^{1}+\mu_{s}^{1} \geq r^{1}\left(s, i, y_{s}\right)+\sum_{t=1}^{*} p\left(t \mid s, i, y_{s}\right) \mu_{i}^{1}$ for all $i \in A_{s}, s \in S$

$$
\alpha_{s}^{2}+\mu_{s}^{2} \geq r^{2}\left(s_{x}, x_{s}, j\right)+\sum_{t=1}^{2} p\left(t \mid s_{3} x_{s}, j\right) \mu_{t}^{2} \text { for } a l l j \in B_{s}, s \in S
$$

d) $\sum_{i=1}^{m_{s}} x_{s}(i)=1, \sum_{j=1}^{n_{s}} y_{s}(j)=1$ for all $s \in S$

e) $x_{s} \geq 0, y_{s} \geq 0$ for all $s \in S$.

Observe that constraints (a) of NLP 6.3 .2 imply that for any feasible solution the objective value is non-negative.

For the $\beta$-discounted criterion feasible solutions with objective value near 0 in NLP 6.2.2, turmed out to correspond with $\beta$-discounted $\epsilon$-equilibria (for specific $>0$ ). Unfortunately, feasible solutions with objective value near 0 in NLP 6.3 .2 do not necessarily correspond with limiting average c-equilibria. This is illustrated in the next example.

\subsubsection{EXAMPLE}

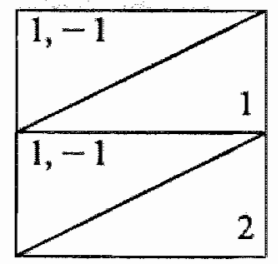

State 1

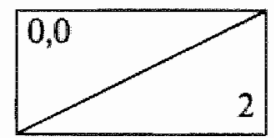

State 2

Let $x=((1-\epsilon, c), 1), \quad y=(1,1), \quad \alpha^{1}=(1,0), \quad \delta^{2}=(-1 / \epsilon, 0) \quad$ and $\quad \alpha^{2}=\delta^{1}=$ $\mu^{1}=\mu^{2}=0$, where $\epsilon>0$. It is easy to verify that $\left(x, y, \alpha^{1}, \alpha^{2}, \delta^{1}, \delta^{2}, \mu^{1}, \mu^{2}\right)$ is a feasible solution of NLP 6.3.2 with objective value $\epsilon$.

It is clear that $(x, y)$ is not a limiting average $\epsilon$-equilibrium for $\epsilon \in(0,1 / 2)$, because $\gamma^{1}(1, x, y)=0<1=\gamma^{1}\left(1, x^{*}, y\right)$ with $x^{*}=((1,0), 1)$.

Since example 6.3 .3 is a zero-sum stochastic game, it also demonstrates that the restriction of NLP 6.3 .2 to zero-sum stochastic games does not yield a program for which near-optimal solutions correspond with stationary limiting average c-optimal strategies.

However for zero-sum stochastic games we present a powerful non-linear program for which feasible solutions with objective value near 0 do indeed correspond with stationary limiting average $\epsilon$-optimal strategies. The non-linear program NLP 6.3 .4 (below) completely characterizes stochastic games with stationary limiting average (e-)optimal strategies. For any stochastic game NLP 6.3.4 finds "best" stationary strategies with respect to the 'distance measure' $d($,$) defined by:$

$$
d\left(x^{*}, y^{*}\right):=\sum_{s=1}^{z}\left[\max _{x} \gamma^{l}\left(s, x, y^{*}\right)-\min _{y} \gamma^{1}\left(s, x^{*}, y\right)\right] .
$$


Thus $d($,$) is a measure for the "distance from optimality" of any pair of sta-$ tionary strategies $\left(x^{*}, y^{*}\right)$.

Notice that for each $s \in S$ it holds that $\max \gamma^{1}\left(s, x, y^{*}\right)-\min \gamma^{\prime}\left(s, x^{*}, y\right)$ is non-negative and: $d\left(x^{*}, y^{*}\right)=0$ if and only if $x^{*}$ and $y^{*}$ are stationary limiting average optimal strategies. If $d\left(x^{*}, y^{*}\right)>0$, then $x^{*}$ and $y^{*}$ are both limiting average $\epsilon$-optimal for some $\epsilon \in\left[0, d\left(x^{*}, y^{*}\right)\right]$.

\subsubsection{THEOREM}

For a zero-sum stochastic game the following results hold.

If there exist $x^{*} \in \sum_{x=1}^{*} \mathbb{R}^{m_{*}}, y^{*} \in \underset{s=1}{x} \mathbb{R}^{n_{1}}, \quad \alpha^{1^{*}}, \alpha^{2^{*}}, \quad \delta^{1^{*}}, \delta^{2^{*}} \in \mathbb{R}^{2}$ such that $\left(x^{*}, y^{*}, \alpha^{1^{*}}, \alpha^{2^{*}}, \delta^{1^{*}}, \delta^{2^{*}}\right)$ is a feasible solution with objective value $\epsilon(\geqslant 0)$ in $N L P$ 6.3 .4 below, then $x^{*}$ and $y^{*}$ are stationary limiting average $\varepsilon$-optimal strategies for the respective players.

Conversely, if $x^{*}$ and $y^{*}$ are stationary limiting average coptimal strategies, then there exist $\alpha^{1^{*}}, \alpha^{2^{*}}, \delta^{1^{*}}, \delta^{2^{*}} \in \mathbb{R}^{2}$ such that $\left(x^{*}, y^{*}, \alpha^{1^{*}}, \alpha^{2^{*}}, \delta^{1^{*}}, \delta^{2^{*}}\right)$ is a feasible solution with objective value less than $2 z \epsilon$ in $N L P$ 6.3.4.

NLP 6.3.4:

variables $\quad x \in \underset{s=1}{x} \mathbb{R}^{m_{s}}, y \in \underset{s=1}{x} \mathbb{R}^{n_{s}}, \alpha^{1}, \alpha^{2}, \delta^{1}, \delta^{2} \in \mathbb{R}^{z}$

minimize $\sum_{s=1}^{z}\left(\alpha_{s}^{1}+\alpha_{s}^{2}\right)$

subject to

a) $\alpha_{s}^{1} \geq \sum_{i=1}^{2} p\left(t \mid s, i, y_{s}\right) \alpha_{i}^{1}$ for all $i \in A_{s}, s \in S$

b) $\alpha_{s}^{1}+\delta_{s}^{1} \geqslant r^{1}\left(s, i, y_{s}\right)+\sum_{i=1}^{z} p\left(t \mid s, i, y_{s}\right) \delta_{t}^{1}$ for all $i \in A_{s}, s \in S$

c) $\alpha_{s}^{2} \geqslant \sum_{t=1}^{z} p\left(t \mid s, x_{s}, j\right) \alpha_{t}^{2}$ for all $j \in B_{s,} s \in S$

d) $\alpha_{s}^{2}+\delta_{s}^{2} \geqslant-r^{1}\left(s, x_{s}, j\right)+\sum_{i=1}^{2} p\left(t \mid s, x_{s}, j\right) \delta_{i}^{2}$ for all $j \in B_{s}, s \in S$

e) $\sum_{i=1}^{m_{4}} x_{s}(i)=1, \sum_{j=1}^{n_{k}} y_{s}(j)=1$ for all $s \in S$

f) $x_{s} \geqslant 0, y_{s} \geqslant 0$ for all $s \in S$.

Proor:

Suppose that $\left(x^{*}, y^{*}, \alpha^{1 *}, \alpha^{2^{*}}, \delta^{1^{*}}, \delta^{2^{*}}\right)$ is feasible in NLP 6.3 .4 with objective value $\epsilon \geqslant 0$.

By constraints (e) and (f) we have $x^{*} \in X$ and $y^{*} \in Y$.

Constraints (a) and (b) imply $a_{s}^{1 *} \geqslant \gamma^{1}\left(s, x, y^{*}\right)$ for all $s \in S, x \in X$ by lemma 1.5.6. Similarly (c) and (d) imply $\alpha_{s}^{2^{*}} \geqslant \gamma^{2}\left(s, x^{*} y\right)=-\gamma^{1}\left(s, x^{*}, y\right)$ for all $s \in S$, $y \in Y$.

Hence $\alpha_{s}^{1}+\alpha_{s}^{2} \geqslant \gamma^{1}\left(s, x, y^{*}\right)-\gamma^{1}\left(s, x^{*}, y\right)$ for all $x \in X, y \in Y, s \in S$.

Especially $\alpha_{s}^{1}+\alpha_{s}^{2} \geqslant \gamma^{1}\left(s, x^{*}, y^{*}\right)-\gamma^{1}\left(s, x^{*}, y^{*}\right)=0$ for all $s \in S$.

Since $\sum_{s=1}^{z}\left(\alpha_{s}^{1}+\alpha_{s}^{2}\right)=c$, we conclude that $\alpha_{s}^{1}+\alpha_{s}^{2} \leqslant €$ for all $s \in S$. 
It follows that for all $s \in S, x \in X, y \in Y$ we have:

$$
\gamma^{l}\left(s_{i}, x^{*}, y\right)+\epsilon \geq \gamma^{\prime \prime}\left(s, x^{*}, y^{*}\right) \geqslant \gamma^{1}\left(s, x, y^{*}\right)-\epsilon
$$

So the strategies $x^{*}$ and $y^{*}$ are limiting average $t$-optimal.

To prove the converse statement, suppose that we have stationary limiting average $\epsilon$-optimal strategies $x^{*} \in X, y^{*} \in Y$. Then constraints (e) and (f) are satisfied.

By solving MDP( $\left.y^{*}\right)$ with respect to the limiting awerage reward criterion (cf. Hordijk \& Kallenberg [1979]) there exist $\alpha^{1^{*}}$ and $\delta^{1^{*}}$ such that (a) and (b) are satisfied. Moreover, we have that $\alpha_{s}^{\mathbf{l}^{*}}=\max \gamma^{\mathrm{l}}\left(s, x, y^{\prime \prime}\right) \leqslant \gamma^{\mathrm{J}}\left(s, x^{*}, y^{*}\right)+\epsilon$ for each $s \in S$. Similarly there exist $\alpha^{2^{*}}$ and $\delta^{2^{*}}$ such that constraints (c) and (d) are satisfied and $\alpha_{s}^{2^{*}}=\max _{y \in Y}\left(-\gamma^{1}\left(s, x^{*}, y\right)\right) \leqslant-\gamma^{1}\left(s, x^{*}, y^{*}\right)+\epsilon$.

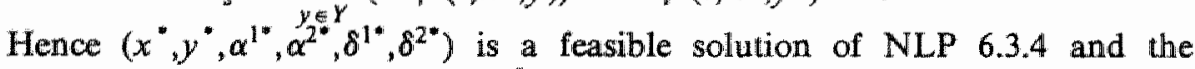
corresponding objective value is $\sum_{s=1}^{z}\left(\alpha_{s}^{1^{*}}+\alpha_{s}^{2^{*}}\right) \leqslant 2 z$.

Theorem 6.3.4 implies that by solving NLP 6.3.4 "best" stationary strategies can be found, as can be seen from the following results.

\subsubsection{CoROllaRY}

If $\left(x^{*}, y^{*}, \alpha^{1^{*}}, \alpha^{2^{*}}, \delta^{1^{*}}, \delta^{2^{*}}\right)$ is a global minimum in NLP 6.3.4 with objective value $\mu \geqslant 0$, then $\mu=d\left(x^{*}, y^{*}\right) \leqslant d(x, y)$ for all $x \in X, y \in Y$.

\section{Proof:}

Let $\left(x^{*}, y^{*}, \alpha^{1^{*}}, \alpha^{2^{*}}, \delta^{1^{*}}, \delta^{2 *}\right)$ be a global minimum in NLP 6.3.4 with objective value $\mu$. By the constraints (a), (b), (c) and (d) it holds that:

$$
\begin{aligned}
& \alpha_{s}^{1^{*}} \geqslant \max _{x \in X} \gamma^{1}\left(s, x, y^{*}\right) \text { for all } s \in S, \\
& \alpha_{s}^{2^{*}} \geq \max _{y \in Y} \gamma^{2}\left(s, x^{*}, y\right)=-\min _{y \in Y} \gamma^{1}\left(s, x^{*}, y\right) \text { for all } s \in S .
\end{aligned}
$$

Since $\left(x^{*}, y^{*}, \alpha^{1^{*}}, \alpha^{2^{*}}, \delta^{1^{*}}, \delta^{2^{*}}\right)$ is a global minimum, equality must hold in all these inequalities. Otherwise, by solving $\operatorname{MDP}\left(x^{*}\right)$ and $\operatorname{MDP}\left(y^{*}\right)$ one could find variables $\alpha^{1}, \alpha^{2}, \delta^{1}, \delta^{2}$ for which equality indeed holds, and hence $\sum_{s=1}^{z}\left(\alpha_{s}^{1}+\alpha_{s}^{2}\right)<\mu$ would contradict the minimality of $\left(x^{*}, y^{*}, \alpha^{1^{*}}, \alpha^{2^{*}}, \delta^{1^{*}}, \delta^{2^{*}}\right)$.

We conclude that $\mu=\sum_{s=1}^{z}\left(\alpha_{s}^{\prime^{*}}+\alpha_{s}^{2^{*}}\right)=d\left(x^{*}, y^{*}\right)$.

For any pair of stationary strategies $(\tilde{x}, \tilde{y})$ one can find $\tilde{\alpha}^{1}, \tilde{\alpha}^{2}, \tilde{\delta}^{1}, \tilde{\delta}^{2}$ such that $\left(\tilde{x}, \tilde{y}, \tilde{\alpha}^{1}, \tilde{\alpha}^{2}, \tilde{\delta}^{1}, \tilde{\delta}^{2}\right)$ is feasible in NLP 6.3.4 and such that $\tilde{\alpha}_{s}^{1}=\max _{x \in X} \gamma^{1}(s, x, \tilde{y})$ and $\tilde{\alpha}_{s}^{2}=-\min _{y \in Y} \gamma^{1}(s, \tilde{x}, y)$ for all $s \in S$. Then $\mu \leqslant \sum_{s=1}^{z}\left(\tilde{\alpha}_{s}^{1}+\tilde{\alpha}_{s}^{2}\right)=d(\tilde{x}, \tilde{y})$ by the minimality of $\mu$. 


\subsubsection{CoRollaRY}

Suppose that the minimum in NLP 6.3.4 does not exist, but that the infimum equals $\mu(\geq 0)$, then for every $c>0$ there exist stationary limiting average $(\mu+c)$ optimal strategies for both players.

\section{Proof:}

For every $\epsilon>0$ there exists $\left(x^{*}, y^{*}, \alpha^{1^{*}}, \alpha^{2^{*}}, \delta^{1^{*}}, \delta^{2^{*}}\right)$ which is feasible in NLP 6.3 .4 with objective value less than $\mu+c$. From theorem 6.3.4 it follows that $x$ " and $y^{*}$ are stationary limiting average $(\mu+\epsilon)$-optimal strategies.

Without proof we state.

\subsubsection{COROLLARY}

For a zero-sum stochastic game there exist stationary limiting average e-optimal strategies for both players and for all $\epsilon>0$, if and only if the infimum of NLP 6.3 .4 is equal to 0.

\subsection{PROGRAMS FOR THE TOTAL REWARD CRITERION.}

From the bad match (section 5.4) it is clear that stationary total equilibria do not necessarily exist; not even in stochastic games for which the limiting average rewards are 0 for all pairs of stationary strategies. Nevertheless we can formulate non-linear programs for which optimal solutions correspond with stationary total equilibria. We make use of the following lemma.

\subsubsection{LEMMA}

For a stochastic game with the property that $\gamma^{k}(x, y)=0$ for all stationary strategies $x$ and $y$, we have: a stationary strategy $x^{*}$ is a total best reply against $y^{*} \in Y$ if and only if there exist $\alpha^{*}, \delta^{*}$ and $\mu^{*} \in \mathbb{R}^{z}$ such that

a) $\alpha_{s}^{*}=r^{1}\left(s, x_{s}^{*}, y_{s}^{*}\right)+\sum_{i=1}^{z} p\left(t \mid s, x_{s}^{*}, y_{s}^{*}\right) \alpha_{i}^{*}$ for all $s \in S$

$$
\alpha_{s}^{*} \geq r^{i}\left(s, i, y_{s}^{*}\right)+\sum_{t=1}^{i} p\left(t \mid s, i, y_{s}^{*}\right) \alpha_{i}^{*} \text { for all } i \in A_{s}, s \in S
$$

b) $\alpha_{s}^{*}+\delta_{s}^{*}=\sum_{i=1}^{z} p\left(t \mid s_{i} x_{s}^{*}, y_{s}^{*}\right) \delta_{i}^{*}$ for all $s \in S$

c) $\alpha_{s}^{*}+\mu_{s}^{*} \geqslant \sum_{i=1}^{z} p\left(t \mid s, i, y_{s}^{*}\right) \mu_{i}^{*}$ for all $i \in A_{s}, s \in S$

ProOF:

The 'if-part follows directly from the lemmas 1.5.7, 1.5.8 and theorem 5.2.5. The "only-if"-part can be shown in a simillar way as the proof for the "only-if' part of theorem 5.3.2.

Lemma 6.4.1 directly leads to the following theorem. 


\subsubsection{THEOREM}

For a general-sum stochastic game with $\gamma^{k}(x, y)=0$ for all $x \in X, y \in Y$, we have: a pair of stationary strategies $\left(x^{*}, y^{*}\right) \in X \times Y$ is a stationary total equilibrium with total reward $\left(\alpha^{1^{*}}, \alpha^{2^{*}}\right)$ if and only if there exist $\delta^{1^{*}}, \delta^{2^{*}}, \mu^{1 *}, \mu^{2^{*}} \in \mathbb{R}^{z}$ such that $\left(x^{*}, y^{*}, \alpha^{1 *}, \alpha^{2^{*}}, \delta^{1 *}, \delta^{2^{*}}, \mu^{1 *}, \mu^{2^{*}}\right)$ is a gloval minimum in the following non-linear program with objective value 0 .

NLP 6.4.2:

variables $\quad x \in \underset{s=1}{x} \mathbb{R}^{m_{n}}, y \in \underset{s=1}{x} Y^{m_{n}}, \alpha^{1}, \alpha^{2}, \delta^{1}, \delta^{2}, \mu^{1}, \mu^{2} \in \mathbb{R}^{z}$

minimize $\sum_{k=1}^{2} \sum_{s=1}^{s=1}\left[\alpha_{s}^{k}-r^{k}\left(s, x_{s}, y_{s}\right)-\sum_{i=1}^{z} p\left(t \mid s, x_{s}, y_{s}\right) \alpha_{i}^{k}\right]$

subject to
a) $\alpha_{s}^{1} \geqslant r^{k}\left(s, i, y_{s}\right)+\sum_{i=1}^{z} p\left(l \mid s, i, y_{s}\right) \alpha_{i}^{1}$ for all $i \in A_{s}, s \in S$
$\alpha_{s}^{2} \geqslant r^{k}\left(s, x_{s}, j\right)+\sum_{i=1}^{z} p\left(t \mid s, x_{s}, j\right) \alpha_{t}^{2}$ for all $j \in B_{s}, s \in S$
b) $\alpha_{s}^{1}+\delta_{s}^{1}=\sum_{t=1}^{z} p\left(t \mid s, x_{s}, y_{s}\right) \delta_{t}^{1}$ for all $s \in S$
$\alpha_{s}^{2}+\delta_{s}^{2}=\sum_{i=1}^{z} p\left(t \mid s, x_{s}, y_{s}\right) \delta_{t}^{2}$ for all $s \in S$
c) $\alpha_{s}^{1}+\mu_{s}^{1} \geqslant \sum_{t=1}^{z} p\left(t \mid s, i, y_{s}\right) \mu_{l}^{1}$ for all $i \in A_{s}, s \in S$
$\alpha_{s}^{2}+\mu_{s}^{2} \geqslant \sum_{t=1}^{z} p\left(t \mid s, x_{s}, j\right) \mu_{t}^{2}$ for all $j \in B_{s}, s \in S$
d) $\sum_{i=1}^{m_{n}} x_{s}(i)=1, \sum_{j=1}^{n_{n}} y_{s}(j)=1$ for all $s \in S$
e) $x_{s} \geqslant 0, y_{s} \geqslant 0$ for all $s \in S$.

Like in the previous sections we wonder whether or not near-optimal solutions of NLP 6.4 .2 correspond with total $\varepsilon$-equilibria. For the total reward criterion we find that, as for the limiting average criterion, there is no such correspondence. This is illustrated by the next example.

\subsubsection{EXAMPLE}

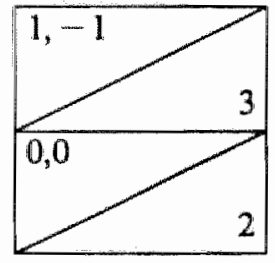

State 1

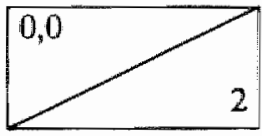

State 2

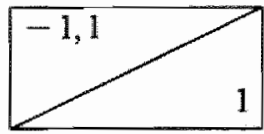

State 3

Let $x=((1-\epsilon, \epsilon), 1,1), \quad 0<\epsilon<1, \quad y=(1,1,1), \quad \alpha^{1}=(1,0,0), \quad \delta^{1}=(1 / \epsilon, 2 / \epsilon, 1 / \epsilon)$, $\alpha^{2}=(0,0,1), \quad \delta^{2}=(1,1 / c, 0)$ and let $\mu^{1}=\mu^{2}=(0,0,0)$. Furthermore let 
$x^{*}=((1,0), 1,1)$. Then $\left(x, y, \alpha^{1}, \alpha^{2}, \delta^{1}, \delta^{2}, \mu^{1}, \mu^{2}\right)$ is a feasible solution of NLP 6.4.2 with objective value $c_{\text {. Howerer }} \gamma_{T}^{1}(1, x, y)=0<1 / 2=\gamma_{T}^{1}\left(1, x^{*}, y\right)$ and hence $(x, y)$ is no total $\varepsilon$-equilibrium for $c \in(0,1 / 2)$.

Since example 6.4 .3 is a zero-sum stochastic game we conclude that the restriction of NLP 6.4.2 to zero-sum stochastic games, does not yield a program for which near-optimal solutions correspond with stationary total e-optimal strategies. Nevertheless we present a non-linear program which has this property.

\subsubsection{THEOREM}

For a zero-sum stochastic game with $\gamma^{k}(x, y)=0$ for all $x \in X, y \in Y$, we have:

If there exist $x^{*} \in \underset{s=1}{X} \mathbb{R}^{m_{*}}, \quad y^{*} \in \underset{s=1}{\times} \mathbb{R}^{n_{*}}, \quad \alpha^{1^{*}}, \alpha^{2^{*}}, \delta^{1^{*}}, \delta^{2^{*}} \in \mathbb{R}^{\bar{*}}$ such that $\left(x^{*}, y^{*}, \alpha^{1^{*}}, \alpha^{2^{*}}, \delta^{1^{*}}, \delta^{2 *}\right)$ is a feasible solution with objective value $\epsilon(\geqslant 0)$ in NLP 6.4 .4 below, then $x^{*}$ and $y^{*}$ are stationary total coptimal strategies for the respective players.

Conversely, if $x^{*}$ and $y^{*}$ are stationary total c-optimal strategies, then there exist $\alpha^{1^{*}}, \alpha^{2^{*}}, \delta^{1^{*}}, \delta^{2^{*}} \in \mathbb{R}^{2}$ such that $\left(x^{*}, y^{*}, \alpha^{1^{*}}, \alpha^{2^{*}}, \delta^{1^{*}}, \delta^{2^{*}}\right)$ is a feasible solution with objective value less than $2 z e$ in $N L P$ 6.4.4.

NLP 6.4.4:

variables $x \in \underset{s=1}{x} \mathbb{R}^{m_{4}}, y \in \underset{s=1}{x} \mathbb{R}^{m_{s}}, \alpha^{1}, \alpha^{2}, \delta^{1}, \delta^{2} \in \mathbb{R}^{2}$

minimize $\sum_{s=1}^{z}\left(\alpha_{s}^{1}+\alpha_{s}^{2}\right)$

subject to

a) $\alpha_{s}^{1} \geqslant r^{1}\left(s, i, y_{s}\right)+\sum_{t=1}^{z} p\left(t \mid s, i, y_{s}\right) \alpha_{i}^{1}$ for all $i \in A_{s}, s \in S$

$$
\alpha_{s}^{2} \geqslant-r^{1}\left(s, x_{s}, j\right)+\sum_{i=1}^{z} p\left(t \mid s_{y}, x_{s}, j\right) \alpha_{t}^{2} \text { for all } j \in B_{s}, s \in S
$$

b) $\alpha_{s}^{1}+\delta_{s}^{1} \geqslant \sum_{i=1}^{z} p\left(t \mid s, i, y_{s}\right) \delta_{1}^{1}$ for all $i \in A_{s}, \mathfrak{s} \in S$

$$
\alpha_{s}^{2}+\delta_{s}^{2} \geqslant \sum_{i=1}^{z} p\left(t \mid s, x_{s}, j\right) \delta_{i}^{2} \text { for all } j \in B_{s}, s \in S
$$

c) $\sum_{i=1}^{m_{s}} x_{s}(i)=1, \sum_{j=1}^{n_{s}} y_{s}(j)=1$ for all $s \in S$

d) $x_{s} \geqslant 0, y_{s} \geqslant 0$ for all $s \in S$.

\section{ProOF:}

Suppose that $\left(x^{*}, y^{*}, \alpha^{l^{*}}, \alpha^{2^{*}}, \delta^{1 *}, \delta^{2^{*}}\right)$ is feasible in NLP 6.4.4 with objective value $\epsilon$. By constraints (c) and (d): $x^{*} \in X, y^{*} \in Y$. By constraints (a) and (b) we have, using lemma 1.5.8, that:

$\gamma_{T}^{\mathbb{1}}\left(s, x, y^{*}\right) \leqslant \alpha_{s}^{1^{*}}$ and $-\gamma_{T}^{1}\left(s, x^{*}, y\right)=\gamma_{T}^{2}\left(s, x^{*}, y\right) \leqslant \alpha_{s}^{2^{*}}$ for all $x \in X, y \in Y, s \in S$. Hence $\gamma_{T}^{1}\left(s, x, y^{*}\right)-\gamma_{T}^{1}\left(s, x^{*}, y\right) \leqslant \alpha_{s}^{1^{*}}+\alpha_{s}^{2^{*}}$ for all $x \in X, y \in Y, s \in S$.

Especially $\alpha_{s}^{1^{*}}+\alpha_{s}^{2^{*}} \geqslant \gamma_{T}^{1}\left(s_{*} x^{*}, y^{*}\right)-\gamma_{T}^{3}\left(s_{s} x^{*}, y^{*}\right)=0$ for all $s \in S$. Since $\sum_{s=1}^{i}\left(\alpha_{s}^{1^{*}}+\alpha_{s}^{2^{*}}\right)=c$ we conclude that $\epsilon \geqslant \alpha_{s}^{1^{*}}+\alpha_{s}^{2^{*}} \geqslant 0$ for all $s \in S$. 
Hence for all $s \in S, x \in X$ and $y \in Y$ we have:

$$
\gamma_{T}^{l}\left(s, x^{*}, y\right)+\epsilon \geqslant \gamma_{\Gamma}^{l}\left(s, x^{*}, y^{*}\right) \geqslant \gamma_{T}^{1}\left(s, x, y^{*}\right)-\epsilon,
$$

which means that $x^{*}$ and $y^{*}$ are stationary total $t$-optimal strategies.

Conversely, if $x^{\prime \prime}$ and $y^{\prime \prime}$ are stationary total $\epsilon$-optimal strategies, then constraints (c) and (d) are satisfied. By solving $\operatorname{MDP}\left(y^{*}\right)$ there exist $\alpha^{2^{*}}$ and $\delta^{2^{*}}$ such that (a) and (b) for player 2 are satisfied (cf. lemma 6.4.1). Similarly one can find $\alpha^{1 *}$ and $\delta^{1^{*}}$ by solving $\operatorname{MDP}\left(x^{*}\right)$. Then $\left(x^{*}, y^{*}, \alpha^{1{ }^{*}}, \alpha^{2^{*}}, \delta^{1^{*}}, \delta^{2^{*}}\right)$ is feasible in NLP 6.4.4. By lemma 6.4.1 we have for all $s \in S$ :

$$
\begin{aligned}
& \alpha_{s}^{1^{*}}=\max _{\forall \in X} \gamma_{T}^{l}\left(s, x, y^{*}\right) \leqslant \gamma_{T}^{1}\left(s, x^{*}, y^{*}\right)+\epsilon \text { and } \\
& \alpha_{s}^{2^{*}}=\max _{y \in Y} \gamma_{T}^{2}\left(s, x^{*}, y\right) \leqslant \gamma_{T}^{2}\left(s, x^{*}, y^{*}\right)+\epsilon=-\gamma_{T}^{1}\left(s, x^{*}, y^{*}\right)+\epsilon .
\end{aligned}
$$

It should be observed that NLP 6.4.4 finds 'best' stationary strategies for the total reward criterion.

Here a 'best' pair of stationary strategies is a pair $\left(x^{*}, y^{*}\right)$ such that

$d_{T}\left(x^{*}, y^{*}\right):=\sum_{s=1}^{z}\left[\max _{x \notin X} \gamma_{T}^{1}\left(s, x, y^{*}\right)-\min _{y \in Y} \gamma_{T}^{1}\left(s, x^{*}, y\right)\right]$ is (near-)minimal.

Without proofs we formulate some implications of theorem 6.4.4. They can be proved in a similar way as the corresponding results for the limiting average reward criterion were proved.

\subsubsection{COROLLARY}

If $\left(x^{*}, y^{*}, \alpha^{1 *}, \alpha^{2 *}, \delta^{1 *}, \delta^{2 *}\right)$ is a global minimum in NLP 6.4.4 with objective value $\mu \geqslant 0$, then $\mu=d_{T}\left(x^{*}, y^{*}\right) \leqslant d_{T}(x, y)$ for all $x \in X, y \in Y$.

\subsubsection{COROLLARY}

Suppose that the minimum in NLP 6.4.4 does not exist, but that the infimum equals $\mu(\geq 0)$, then for every $c>0$ there exist stationary total $(\mu+c)$-optimal straregies for both players.

\subsubsection{Corollary}

For a zero-sum stochastic game there exist stationary total e-optimal strategies for both players and for all $\varepsilon>0$, if and only if the infimum of NLP 6.4 .4 is equal to 0 . 


\section{References}

R.J. Aumann [1964]: Mixed and behavior strategies in infinite extensive games. In: Advances in Game Theory, Annals of Mathematical Studies 52, Princeton University Press, 627-650.

R.J. Aumann [1981]: Survey of repeated games. In: Essays in Game Theory and Mathematical Economics in Honor of Oskar Morgenstern, Bibliographisches Institüt, Mannheim, 11-42.

R. Bellman [1952]: On the theory of dynamic programming. Proceedings of the National Academy of Sciences U.S.A. 38, 716-719.

R. Bellman [1957]: Dynamic Programming. Princeton University Press.

T. Bewley \& E. Kohlberg [1976]: The asymptotic theory of stochastic games. Mathematics of Operations Research 1, 197-208.

T. Bewley \& E. Kohlberg [1978]: On stochastic games with optimal stationary strategies. Mathematics of Operations Research 3, 104-125.

P. Billingsley [1979]: Probability and Measure. John Wiley \& Sons, New York.

D. Blackwell [1962]: Discrete dynamic programming. Annals of Mathematical Statistics 33, 719-726.

D. Blackwell \& T.S. Ferguson [1968]: The big match. Annals of Mathematical Statistics 39, 159-163.

C. Derman [1970]: Finite State Markovian Decision Processes. Academic Press, New York.

H. Everett [1957]: Recursive games. In: M. Dresher, A.W. Tucker \& P. Wolfe (eds.), Contributions to the Theory of Games III, Annals of Mathematical Studies 39, Princeton University Press, 47-78.

A. Federgruen [1978]: On n-person stochastic games with denumerable state space. Advances in Applied Probability 10, 452-471.

J.A. Filar [1981-a]: A single-loop stochastic game which one player can terminate. Opsearch 18, 185-203.

J.A. Filar [1981-b]: Ordered field property for stochastic games when the player who controls transitions changes from state to state. Journal of Optimization Theory and Applications 34, 503-515.

J.A. Filar [1984]: On stationary equilibria of a single controller stochastic game. Mathematical Programming 30, 313-325.

J.A. Filar [1986]: Quadratic programming and the single controller stochastic game. Journal of Mathematical Analysis and Applications 113, 136-147.

J.A. Filar \& T.E.S. Raghavan [1984]: A matrix game solution of the single controller stochastic game. Mathematics of Operations Research 9, 356-362.

J.A. Filar \& T.A. Schultz [1986]: Nonlinear programming and stationary strategies in stochastic games. Mathematical Programming 35, $243-247$. 
J.A. Filar \& T.A. Schultz [1987]: Bilinear programming and structured stochastic games. Journal of Optimization Theory and Applications 53, 85-104.

J.A. Filar, T.A. Schultz, F. Thuijsman \& O.J. Vrieze [1987]: Nonlinear programming and stationary equilibria in stochastic games. Report 87-18, Department of Mathematics, University of Maryland Baltimore County ${ }_{*}$ Baltimore (Conditionally accepted by Mathematical Programming).

J.A. Filar \& O.J. Vrieze [1989]: Weighted reward criteria in competitive Markov decision processes. Technical report, Department of Mathematics, University of Maryland Baltimore County, Baltimore.

A.M. Fink [1964]: Equilibrium in a stochastic n-person game. Journal of Science of Hiroshima University, Series A-I 28, 89-93.

D. Gillette [1957]: Stochastic games with zero stop probabilities. In: M. Dresher, A.W. Tucker \& P. Wolfe (eds.), Contributions to the Theory of Games III, Annals of Mathematical Studies 39, Princeton University Press, 179-187.

A.J. Hoffman \& R.M. Karp [1966]: On nonterminating stochastic games. Management Science 12, 359-370.

A. Hordijk \& L.C.M. Kallenberg [1979]: Linear programming and Markov decision chains. Management Science 25, 352-362.

A. Hordijk \& L.C.M. Kallenberg [1981]: Linear programming and Markov games II. In: O. Moeschlin, D. Pallaschke (eds.), Game Theory and Mathematical Economics, North Holland Publishing Company, Amsterdam, 307-320.

A. Hordijk, O.J. Vrieze \& G.L. Wanrooij [1983]: Semi-Markov strategies in stochastic games. International Journal of Game Theory 12, 81-89.

L.C.M. Kallenberg [1983]: Linear Programming and Finite Markovian Control Problems. Mathematical Centre Tract 148, Centre for Mathematics and Computer Science, Amsterdam.

J. Kemeny \& J. Snell [1960]: Finite Markov Chains. Van Nostrand, Princeton.

E. Kohlberg [1974]: Repeated games with absorbing states. Annals of Statistics $2,724-738$.

A. Kolmogorow [1933]: Grundbegriffe der Wahrscheinlichkeitsrechnung. Ergebnisse der Mathematik 2, no. 3, Springer Verlag, Berlin.

D. Krass, J.A. Filar \& S. Sinha [1987]: A weighted Markov decision process. Technical report, Department of Mathematics, University of Maryland Baltimore County, Baltimore.

T.M. Liggett \& S.A. Lippman [1969]: Stochastic games with perfect information and time average payoff. SIAM Review 11, 604-607.

O.L. Mangasarian \& H. Stone [1964]: Two-person non-zerosum stochastic games and quadratic programming. Journal of Mathematical Analysis and Applications $9,348-355$.

J.F. Mertens [1986]: Repeated games. CORE reprint 8624, Center for Operations Research and Econometrics, Université Catholique de Louvain, Louvainla-Neuve.

J.F. Mertens \& A. Neyman [1981]: Stochastic games. International Journal of Game Theory 10, 53-66.

J. Nash [1951]: Non-cooperative games. Annals of Mathematics 54, 286-295. 
J. von Neumann [1928]: Zur Theorie der Gesellschaftsspiele. Mathematische Annalen 100, 295-320.

J. von Neumann \& O. Morgenstern [1944]: Theory of Games and Economic Behavior. Princeton University Press.

A. Neyman [1986]: Private communication.

M. Orkin [1972]: Recursive matrix games. Journal of Applied Probability 9, 813-820.

T. Parthasarathy \& T.E.S. Raghavan [1981]: An orderfield property for stochastic games when one player controls transition probabilities. Journal of Optimization Theory and Applications 33, 375-392.

T. Parthasarathy \& M.A. Stern [1977]: Markov Games, a survey. In: E. Roxin, P. Lieu \& R. Sternberg (eds.), Differential Games and Control Theory 2, Marcel Dekker, New York, 1-46.

T. Parthasarathy, S.H. Tijs \& O.J. Vrieze [1984]: Stochastic games with state independent transitions and separable rewards. In: G. Hammer \& D. Pallaschke (eds.), Selected Topics in Operations Research and Mathematical Economics, Springer Verlag, Berlin 262-271.

T.E.S. Raghavan \& J.A. Filar [1989]: Algorithms for stochastic games a survey. Report, Department of Mathematics, University of Illinois, Chicago.

T.E.S. Raghavan, S.H. Tijs \& O.J. Vrieze [1985]: On stochastic games with additive reward and transition structure. Journal of Optimization Theory and Applications 47, 451-464.

P.D. Rogers [1969]: Non-zerosum stochastic games. Ph.D. thesis, Report ORC 69-8, Operations Research Center, University of California, Berkeley.

U.G. Rothblum [1979]: Solving stopping stochastic games by maximizing a linear function subject to quadratic constraints. In: O. Moeschlin \& D. Pallaschke (eds.), Game Theory and Related Topics, North-Holland Publishing Company, Amsterdam, 103-105.

T.A. Schultz [1987]: Mathematical programming and stochastic games. Ph.D. thesis, Johns Hopkins University, Baltimore.

L.S. Shapley [1953]: Stochastic games. Proceedings of the National Academy of Sciences U.S.A. 39, 1095-1100.

L.S. Shapley \& R.N. Snow [1950]: Basic solutions of discrete games. In: H.W. Kuhn \& A.W. Tucker (eds.), Contributions to the Theory of Games I, Annals of Mathematical Studies 24, Princeton University Press, 27-35.

M.J. Sobel [1971]: Noncooperative stochastic games. Annals of Mathematical Statistics 42, 1930-1935.

M.J. Sobel [1981]: Myopic solutions of Markov decision processes and stochastic games. Operations Research 29, 995-1009.

S. Sorin [1986]: Asymptotic properties of a non-zerosum stochastic game. International Journal of Game Theory 15, 101-107.

S. Sorin [1988]: Repeated games with complete information. CORE discussion paper 8822, Center for Operations Research and Econometrics, Université Catholique de Louvain, Louvain-la-Neuve.

M.A. Stern [1975]: On stochastic games with limiting average payoff. Ph.D. thesis, University of Illinois, Chicago. 
M. Takahashi [1964]: Equilibrium points of stochastic noncooperative n-person games. Journal of Science of Hiroshima University, Series A-1 28, 95-99.

A. Tarskil [1951]: A Decision Method for Elementary Algebra and Geometry. Second edition, revised, University of California Press, Berkeley and Los Angeles.

F. Thuijsman [1987]: Non-zerosum stochastic games. In: H.J.M. Peters \& O.J. Vrieze (eds.), Surveys in Game Theory and Related Topics, CWI-tract 39, Centre for Mathematics and Computer Science, Amsterdam, 133-161.

F. Thuijsman \& 0.J, Vrieze [1987]: The bad match, a total reward stochastic game. OR Spektrum 9, 93-99.

F. Thuijsman \& O.J. Vrileze [1989-a]: Easy initial states in stochastic games. Forthcoming in: T.E.S. Raghavan, T.S. Ferguson, O.J. Vrieze \& T. Parthasarathy (eds.), Stochastic Games and Related Topics in Honor of L.S. Shapley, Kluwer Academic Publishers, Dordrecht.

F. Thuijsman \& O.J. Vrieze [1989-b]: €-Easy initial states in zero-sum stochastic games. Report M89-03, Department of Mathematics, University of Limburg, Maastricht (forthcoming).

F. Thuijsman \& O.J. Vrieze [1989-c]: Strong initial states in general-sum stochastic games. Report M89-04, Department of Mathematics, University of Limburg, Maastricht (forthcoming).

S.H. Tijs \& O.J. Vrieze [1986]: On the existence of easy initial states for undiscounted stochastic games. Mathematics of Operations Research 11, 506513.

O.J. Vrieze [1981]: Linear programming and undiscounted stochastic games in which one player controls transitions. OR Spektrum 3, 29-35.

O.J. Vrieze [1983]: Discounted stochastic games and mathematical programming. Report 8352, Department of Mathematics, Catholic University, Nijmegen.

O.J. Vrieze [1987-a]: Stochastic Games with Finite State and Action Spaces. CWI-tract 33, Centre for Mathematics and Computer Science, Amsterdam.

O.J. Vrieze [1987-b]: Zero-sum stochastic games. In: H.J.M. Peters \& O.J. Vrieze (eds.), Surveys in Game Theory and Related Topics, CWI-tract 39, Centre for Mathematics and Computer Science, Amsterdam, 103-162.

O.J. Vrieze \& F. Thui]sman [1987]: Stochastic games and optimal stationary strategies, a survey. In: W. Domschke, W. Krabs, J. Lehn \& P. Spellucci (eds.), Methods of Operations Research 57, 513-529.

O.J. Vrieze \& F. Thuijsman [1989]: On equilibria in repeated games with absorb. ing states. Forthcoming in International Journal of Game Theory.

O.J. Vrieze \& S.H. Tijs [1980]: Relations between the game parameters, value and optimal strategy spaces in stochastic games and construction of games with given solution. Journal of Optimization Theory and Applications 31, 501-513.

O.J. Vrieze, S.H., Tijs, T.E.S. Raghavan \& J.A. Filar [1983]: A finite algorithm for the switching control stochastic game. OR Spektrum 5, 15-24.

D.V. Widder [1941]: The Laplace Transform. Princeton University Press.

P. Wolfe [1956]: Determinateness of polyhedral games. In: H.W. Kuhn, A.W. Tucker (eds.), Linear Inequalities and Related Systems I, Annals of Mathematical Studies 38, Princeton University Press, Princeton, 195-198. 


\section{Author index}

Aumann 5, 8, 23, 99

Bellman 1, 99

Bewley 3, 4, 20, 21, 37, 40, 43, 69, $73,75,99$

Billingsley 32,99

Blackwell 3, 4, 12, 16, 19, 21, 62, 74, $79,85,91,99$

Derman 72, 99

Everett 3,99

Federgruen 3, 59, 99

Ferguson 3, 4, 19, 21, 62, 74, 79, 85, 99

Filar 3, 4, 5, 62, 69, 70, 88, 99, $100,101,102$

Fink 2, 3, 4, 22, 100

Gillette $2,3,10,19,59,100$

Hoffman $3,59,100$

Hordijk 3, 16, 88, 91, 94, 100

Kallenberg 3, 72, 88, 91, 94, 100

Karp 3,59, 100

Kemeny 12, 13, 29, 100

Kohlberg 3, 4, 20, 21, 37, 40, 43, 62, $69,73,75,99,100$

Kolmogorov 10, 100

Krass 70,100

Liggett 4,100

Lippman 4, 100

Mangasarian 87, 100

Mertens 4, 5, 21, 45, 75, 100
Morgenstern 1, 101

Nash 2, 22, 101

Neyman 4, 21, 45, 71, 100, 101

Neumann von 1, 18, 101

Orkin 3, 101

Parthasarathy $3,4,5,60,101$

Raghavan 3, 4, 5, 99, 101, 102

Rogers 3, 4, 23, 59, 88, 101

Rothblum 88, 90, 101

Schultz 3, 4, 69, 70, 76, 88, 99, 100, 101

Shapley $1,2,3,9,17,18,22,101$

Sinha 100

Snell $12,13,29,100$

Snow 76, 101

Sobel 3, 4, 23, 59, 60, 69, 101

Sorin 4, 5, 24, 47, 62, 101

Stern 3, 5, 101, 102

Stone 87,100

Takahashi $4,23,102$

Tarski 20, 102

Thuijsman 4, 5, 10, 27, 45, 62, 69, 100,102

Tijs 4, 5, 19, 27, 37, 101, 102

Vrieze $3,4,5,10,19,27,37,43,45$, $62,69,70,75,76,88,100,101$, 102

Wanrooij 100

Widder 72,102

Wolfe 76,102 



\section{Subject index}

absorbing pair of strategies 63 absorbing state 62

action space 6

almost stationary limiting average e-equilibrium 4, 31

asymptotic properties 20

bad match 79

behavior strategy 8

best reply 15

best stationary strategy 93,98

big match 3,19

bimatrix game $2,22,87$

carrier 11

characterizations for stationary solutions 4, 69, 88

decision epoch 79

$\beta$-discounted reward 2,9

dynamic programming 1

(є-)easy initial state 27,37

(e-)equilibrium 2,22

evaluation criteria 8

expected payoff 9,11

general-sum stochastic game 2, 22

history 7,8

history dependent strategy 3,8

initial state 6

limit properties 28

limiting average reward 2,10

linear program 87

Markov chain 12

Markov decision process 16

Markov strategy 7

mathematical programming 87

matrix game $1,18,87$

mixed action 7

Nash-equilibrium 2, 22

non-cooperative game 1 non-linear program 87

optimal mixed action 18

(€-)optimal strategy 2,18

payoff 9

payoff function 6

play 6

playing against a fixed stationary strategy 15

polyhedral game 76

property $P 75$

pure action 7

pure strategy 8

repeated game 3

repeated game with absorbing

states 62

retaliation strategy 23

reward 9

Shapley-equation 18

SIT stochastic game 60

special classes 59

stage 1,6

state space 6

state independent transitions 60

stochastic game 1, 6

strattegy 7

strong initial state $27,30,31$

threat-point 23

total reward 2, 10,69

transition matrix 11

transition probability map 6

unichain stochastic games 59

uniform $\beta$-discounted optimal strategy 73

value 1,18

zero-sum stochastic game 1,17

$k$-zero-sum stochastic game 23 



\section{Optimaliteit en Evenwichten in Stochastische Spelen}

\section{Samenvatting}

Dit proefschrift handelt over tweepersoons stochastische spelen met eindige toestandsruimte en eindige aktieruimten. Dit zijn niet-coöperatieve dynamische spelen waarbij het spelproces zich, via een aftelbaar oneindig aantal tijdstippen, afspeelt over een eindig aantal toestanden. Ieder van de toestanden kan als begintoestand dienen voor het spelproces. Op ieder tijdstip bevindt het spelproces zich in precies één van die toestanden en moeten twee personen, speler 1 en speler 2 genoemd, gelijktijdig en onafhankelijk van elkaar, elk een aktie kiezen uit een bij die toestand behorende eindige aktieverzameling. Die toestand en de gekozen akties bepalen voor beide spelers een uitbetaling alsook een kansvector volgens welke het spelproces zich naar een volgende toestand verplaatst. Doel van elk van de spelers is het om zo veel mogelijk te verdienen en dat zonder de mogelijkheid te hebben om bindende afspraken te maken.

Op ieder tijdstip kennen beide spelers niet alleen de toestandsruimte, de aktieruimten en de bijbehorende uitbetalings- en overgangsfunktie, maar ook kennen zij de hele historie op dat tijdstip. Hierbij is de historie op een tijdstip de reeks van toestanden en aktiekeuzen (van beide spelers) volgens welke het spelproces zich tot dan toe heeft afgespeeld. Gebruikmakend van deze informatie hanteren de spelers strategieën, speelplannen, om het spelproces te sturen. Een strategie van een speler legt precies vast voor ieder tijdstip, voor iedere toestand en voor iedere historie, welke aktie die speler moet kiezen. De meest complexe strategieèn zijn historie-afhankelijke strategieèn. Daaronder worden vooral strategieën verstaan waarbij de te nemen aktiekeuzen afhankelijk zijn van, door de tegenstander, in het verleden gekozen akties. De minst complexe strategieèn zijn stationaire strategieèn, waarbij de voorgeschreven aktiekeuzen noch van het tijdstip, noch wan de historie, maar enkel van de toestand afhangen.

De spelers proberen ieder hun verwachte opbrengst te maximaliseren. Hierbij wordt de verwachte opbrengst voor een speler vastgelegd door de manier waarop die speler de oneindige stroom van uitbetalingen interpreteert. Om die oneindige stromen van uitbetalingen te interpreteren gebruiken de spelers een evaluatiecriterium. We gaan ervan uit dat beide spelers hetzelfde criterium gebruiken. In dit proefschrift worden stochastische spelen bestudleerd voor drie verschillende evaluatiecriteria, te weten het $\beta$-verdisconteerde opbrengstencriterium, het limiet-gemiddelde opbrengstencriterium en het totale opbrengstencriterium.

In het algemeen zullen de spelers verschillende preferenties hebben over de toestanden en de daarin, onafhankelijk van elkaar, te kiezen aktieparen. Fen interessante klasse van stochastische spelen wordt gevormd door de 
zogenaamde nulsom stochastische spelen waarbij, voor iedere toestand en voor ieder paar akties dat daar gekozen kan worden, geldt dat speler 2 datgene betaalit wat speler $1 \mathrm{krijgt}$. In dergelijke spelen hebben de spelers strikt tegengestelde belangen. De centrale vraag in nulsom stochastische spelen is de vraag of er (voor iedere starttoestand) een bepaald bedrag $v$ bestaat, waarvoor geldt dat speler $\mathbb{1}$ een strategie heeft waarmee hij ervoor kan zorgen dat zijn verwachte opbrengst minstens $v$ zal zijn en waarvoor gelijktijdig geldt dat speler 2 een strategie heeft waarmee die ervoor kan zorgen dat de verwachte opbrengst van speler 1 hoogstens $v$ zal zijn. Mogelijk kunnen de spelers zich $v$ slechts op $\epsilon$ na garanderen $(\epsilon>0)$. Zo'n bedrag $w$ wordt dan de waarde (voor die starttoestand) genoemd en de bijbehorende strategieën heten ( $\epsilon$-)optimale strategieën. Hierbij zijn waarde en $\epsilon$-optimale strategieën natuurlijk afhankelijk van het, door beide spelers gehanteerde, evaluatiecriterium.

In niet-nulsom stochastische spelen hebben de begrippen 'waarde' en ' $\epsilon$ optimale strategieèn' weinig betekenis. Daar vormen $\epsilon$-evenwichten het gebruikelijke oplossingsconcept. Een e-evenwicht is een paar strategieën met de eigenschap dat geen der spelers meer dan $\epsilon$ kan verdienen door eenzijdig van zijn strategie af te wijken.

In dit proefschrift worden zowel nulsom stochastische spelen als niet-nulsom stochastische spelen bestudeerd, en wel voor lieder van de drie hierboven genoemde evaluatiecriteria.

Voor stochastische spelen ten aanzien van het $\beta$-verdisconteerde opbrengstencriterium is het welbekend dat in het nulsom geval de waarde en stationaire optimale strategieën voor beide spelers altijd bestaan; in het niet-nulsom geval bestaan er altijd stationaire evenwichten.

Ten aanzien van het limiet-gemiddelde opbrengstencriterium bestaat in het nulsom geval de waarde, maar voor beide spelers zullen historie-afhankelijke strategieën in het algemeen onontbeerlijk zijn om $\epsilon$-optimaal te kunnen spelen; in het niet-nulsom geval is de existentie wan $\epsilon$-evenwichten het belangrijkste onopgeloste probleem.

Voor wat het totale opbrengstencriterium betreft zijn er slechts oplossingen bekend voor speciale gestructureerde klassen van stochastische spelen. Ook voor dit laatste criterium zijn historie-afhankelijke strategieën onmisbaar om tot een oplossing te komen.

De indeling van dit proefschrift is als volgt.

In hoofdstuk 1 worden stochastische spelen formeel geïntroduceerd, alsook de erbij behorende oplossingsconcepten. Verder wordt een beknopt overzicht gegeven van historische resultaten die voor de onderwerpen in dit proefschrift van belang zijn. Voorts worden enkele elementaire resultaten afgeleid. Een en ander wordt aan de hand van een aantal voorbeelden toegelicht.

In hoofdstuk 2 wordt aangetoond dat er voor ieder niet-nulsom stochastisch spel een niet-lege verzameling begintoestanden is, waarvoor een 'bijna stationair" limiet-gemiddeld e-evenwicht bestaat. Met een bijna stationair limietgemiddeld $\epsilon$-evenwicht bedoelen we een $\epsilon$-evenwicht, ten aanzien van het limiet-gemiddelde opbrengsten criterium, bestaande uit strategieën van het 
type: gebruik een bepaalde stationaire strategie tenzij het zeer waarschijnlijk is dat je tegenstander afwijkt van een eveneens bepaalde strategie; in dat laatste geval, begin onmiddellijk je tegenstander af te straffen door optimaal te spelen in het gerelateerde nulsom spel dat gegeven wordt door de uitbetalingen aan die tegenstander.

Voor nulsom stochastische spelen laten we zien dat er voor iedere speler een niet-lege verzameling begintoestanden is, van waaruit die speler een stationair limiet-gemiddeld optimale strategie heeft. We geven voldoende voorwarden onder welke beide spelers stationaire limiet-gemiddeld $€$-optimale strategieẽn hebben voor alle begintoestanden met maximale, dan wel minimale, limietgemiddelde waarde. Een voorbeeld laat zien dat er begintoestanden kunnen zijn waarvoor geen der spelers een stationaire limiet-gemiddeld e-optimale strategie heeft.

In hoofdstuk 3 worden de resultaten uit hoofdstuk 2 voor niet-nulsom stochastische spelen uigebreid door voldoende voorwaarden te geven voor het bestaan van een bijna stationair limiet-gemiddeld $\epsilon$-evenwicht voor alle begintoestanden. Deze voldoende voorwaarden worden geformuleerd aan de hand van eigenschappen van een convergente rij van stationaire $\beta$ verdisconteerde evenwichten (waarbij $\beta$ naar 1 gaat).

In hoofdstuk 4 bestuderen we implicaties van de resultaten uit de hoofdstukken 2 en 3 voor een aantal deelklassen van stochastische spelen: stochastische spelen met voor ieder paar stationaire strategieèn slechts éen ergodische klasse, stochastische spelen met toestandsonafhankelijke overgangen, herhaalde spelen met absorberende toestanden.

Hoofdstuk 5 is gewijd aan het totale opbrengstencriterium. We laten zien dat, zelfs wanneer de limiet-gemiddelde waarde 0 is voor iedere toestand, de totale waarde niet hoeft te bestaan. Ook tonen we aan dat zelfs onder de sterkere conditie dat de limiet-gemiddelde waarde 0 is en dat beide spelers stationaire limiet-gemiddeld optimale strategieën bezitten, historie-aflhankelijke strategieën onmisbaar kunnen zijn om totaal c-optimaal te spelen. Onder deze sterkere voorwaarde geven we ook karakteriseringen voor het bestaan van de totale waarde en stationaire totaal optimale strategieën voor beide spelers. Tevens vergelijken we het totale opbrengstencriterium met zowel het $\beta$ verdisconteerde opbrengstencriterium als het limiet-gemiddelde opbrengstencriterium.

In hoofdstuk 6 worden karakteriseringen gegeven voor het bestaan wan stationaire evenwichten (resp. ( $\epsilon$-)optimale strategieën) in niet-nulsom (resp. nulsom) stochastische spelen, aan de hand van oplossingen voor gerelateerde mathematische programmas. Dit wordt gedaan voor elk van de 3 evaluatiecriteria, waarbij we voor het totale opbrengstencriterium veronderstellen dat de limiet-gemiddelde opbrengst 0 is voor alle paren van stationaire strategieèn. 



\section{Curriculum Vitae}

De schrijver van dit proefschrift werd te Arnhem geboren op 13 september 1958. Aldaar volgde hij middelbaar onderwijs an het Christelijk Lyceum en behaalde hij in juli 1977 het VWO-diploma. Vervolgens begon hij aan een studie wiskunde aan de Katholieke Universiteit te Nijmegen. In januari 1982 behaalde hij het MO-A diploma wiskunde en in november van datzelfde jaar verwierf hij het kandidaatsdiploma wiskunde met als bijvakken sterrenkunde en psychologie. Zijn doctoraalstudie richtte zich voornamelijk op de mathematische besliskunde en de speltheorie. Onder begelleiding van de hoogleraren Dr. S.H. Tijs en Dr.ir.drs. O.J. Vrieze schreef hij zijn doctoraalscriptie over stochastische spelen ten aanzien van het totalle opbrengstencriterium. In mei 1985 legde hij het doctoraalexamen af, met als hoofdvak mathematische besliskunde en als bijvakken statistiek en stochastische speltheorie. Tevens verwierf hij de eerstegraads lesbevoegdheid in de wiskunde.

Van juli 1985 tot juli 1989 was hij als wetenschappelijk medewerker in dienst van de Nederlandse Organisatie voor Wetenschappelijk Onderzoek NWO (voorheen ZWO). Onder supervisie van de hoogleraren Dr. S.H. Tijs en Dr.ir.drs. O.J. Vrieze verrichtte hij verder onderzoek op het gebied van de stochastische speltheorie. De belangrijkste resultaten, voortgekomen uit deze studie, zijn beschreven in dit proefschrift. Van juli 1985 tot november 1987 vond dit onderzoek plaats aan het mathematisch instituut van de Katholieke Universiteit te Nijmegen; vanaf november 1987 werd het onderzoek uitgevoerd bij de vakgroep wiskunde van de Faculteit der Algemene Wetenschappen van de Rijksuniversiteit Limburg te Maastricht. $\mathrm{Na}$ juni 1989 zal hij als universitair docent bij deze vakgroep werkzaam zijn. 